Identifying Interactions for Virtual Reality; a Study of Collaboration and Engagement in Different Media

by

\title{
Justin Chin
}

A thesis submitted to the Faculty of Graduate and Postdoctoral Affairs in partial fulfillment of the requirements for the degree of

\author{
Master of Design \\ in \\ Design \\ Carleton University
Ottawa, Ontario
}

(C) 2019

Justin Chin 


\begin{abstract}
Designers use many methods and techniques to develop ideas and quickly generate concepts. Often these include collaboration and engagement with post-it notes. Technology has enabled exercises using post-it notes to be accessed beyond the physical realm, as digital forms of the post-it-note exist in software applications. This study focused on the experience of using collaborative tools for design thinking practices using post-it notes in face-to-face and digital activities in order to discover common features that could be applied in the design of virtual reality design thinking tools. It found that some common activities in face-to-face collaborative design thinking interactions among designers, such as navigation, selection, manipulation, text input, among others differed from interactions in 2D and 3D Virtual Environments. Study participants worked on design thinking exercises in three different teams using three different types of media (face-to-face, 2D digital, and 3D virtual reality). The teams' behaviours were analyzed to identify and classify the different kinds of interactions that took place. Once these interactions were identified, common and unique gestures were grouped for further analysis. Patterns of activity intensity were identified within and across teams, providing the opportunity to make recommendations about features that could be improved to better suit design collaboration and the user experience in virtual reality.
\end{abstract}

Keywords: virtual reality, brainstorming, design thinking, collaboration, interactions. 


\section{Acknowledgments}

I would like to express my thanks to Professor Lois Frankel and Professor Rob Teather for their guidance and encouragement. Their willingness to give their time so generously has been very much appreciated; without their support this project would have been much more difficult. I would like to thank my colleagues, the staff and faculty of the School of Industrial Design, for sharing the amusement, excitement and joy of the design community.

Thanks to all participants for being available and agreeing to participate in this study. I would also like to thank IBM for allowing some of their designers to participate and visit Carleton to conduct the study. A big thank you to Nureva, in particular Roger O'Connor who allowed me to borrow some equipment and software licenses to complete the study. For Rob Teather and the HCI Department allowing me access to their lab and equipment to conduct the study. 


\section{Preface}

When the researcher first started his journey into the master's program, he was initially interested in using VR (virtual reality) as a design tool. During his summer internship he worked at Nureva, a company that specializes in technologies that enable people to work together remotely on-line, such as audio-conferencing systems and visual collaboration tools. During his time there, he found the digital method of post-it notes very useful and easy to get others to join in without having to provide instructional materials. Given his initial interest in VR, he was inspired to explore how the use of post-it notes for brainstorming could be further translated into 3D workspaces, similar to Nureva's 2D Span software, but in Virtual Reality.

This led to studying multi-user VR experiences and collaborative efforts by researchers, and even the gaming community. While collaborative efforts have been tried before, nothing was quite like he imagined it could be. For example, in many gaming applications, there was a focus on the social aspect of having many people in the VR platform at the same time, however this effort was more for entertainment than collaboration. Therefore, it seemed like there would be an opportunity to study what kinds of tools, if any, could be useful for collaboration in VR. 


\section{Table of Contents}

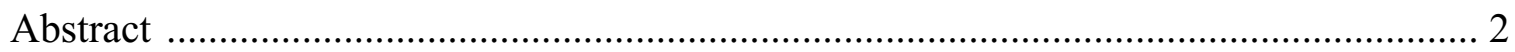

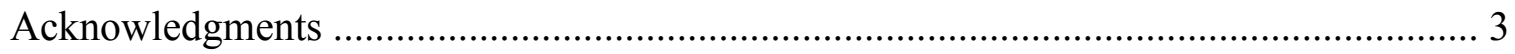

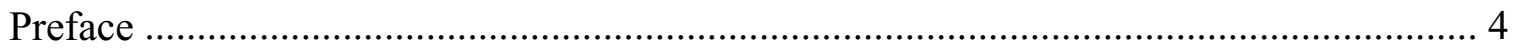

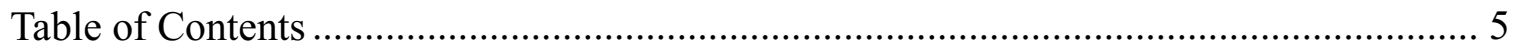

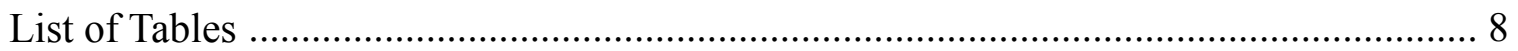

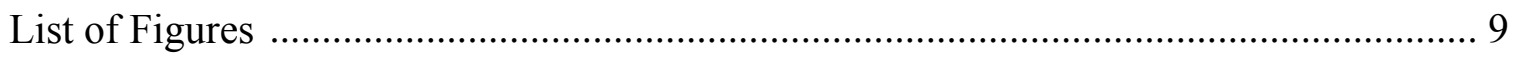

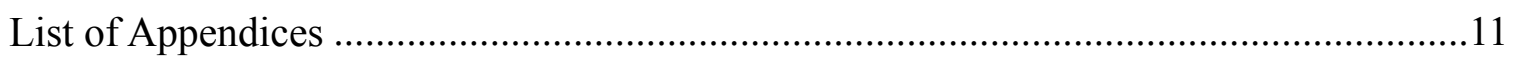

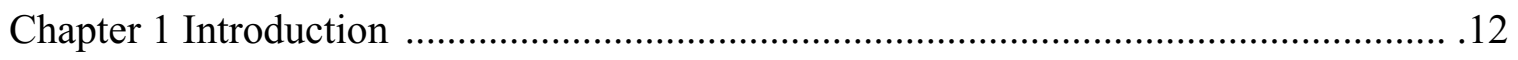

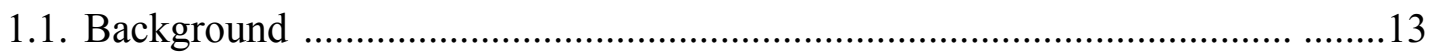

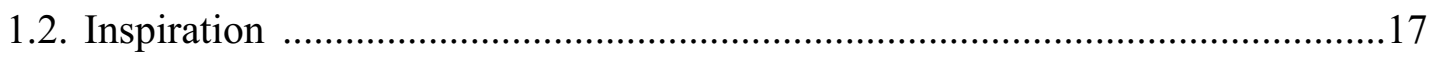

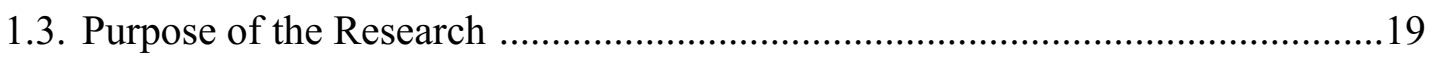

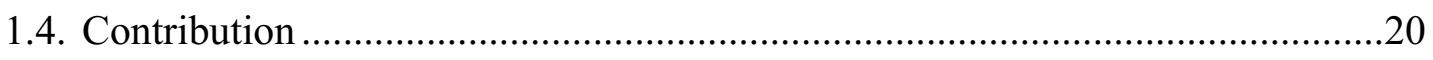

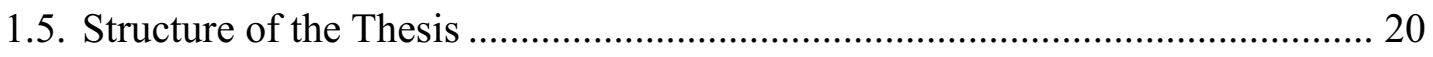

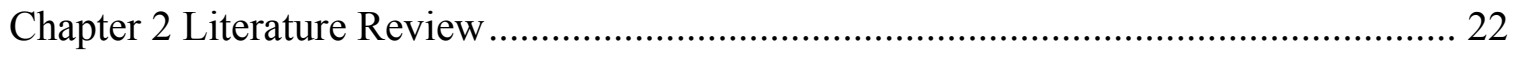

2.1. How Behaviours Differ in 2D vs. 3D .......................................................... 22

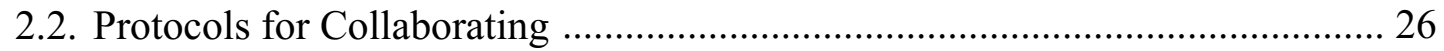

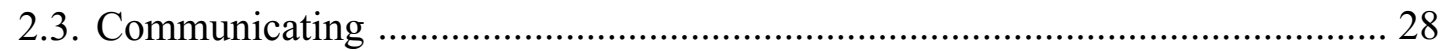

2.4. The Nature of the Environment or Context ................................................... 29

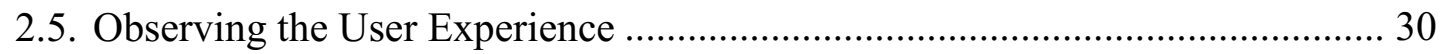

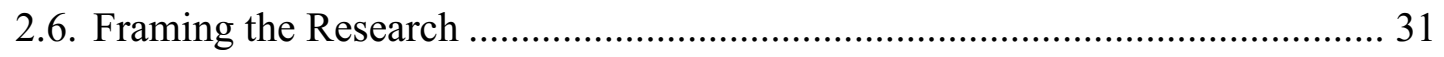

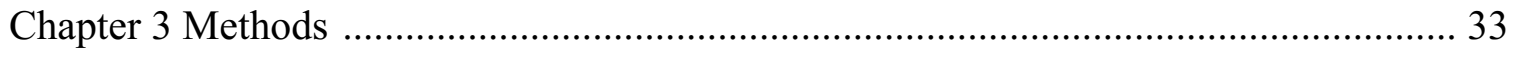

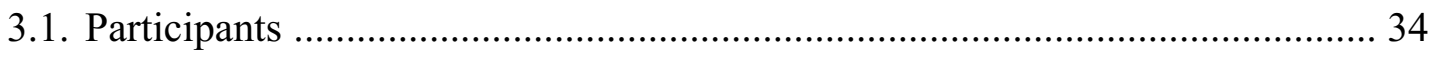

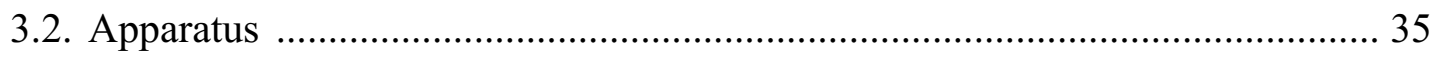

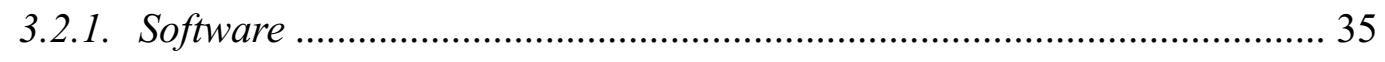

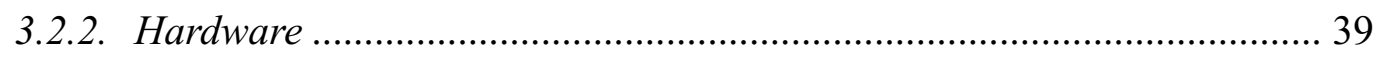

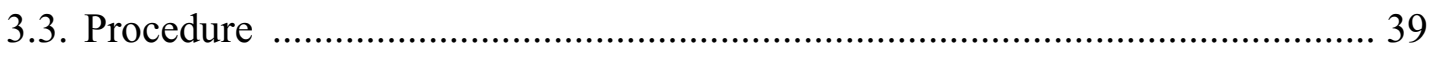




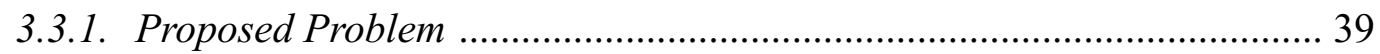

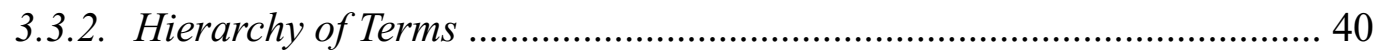

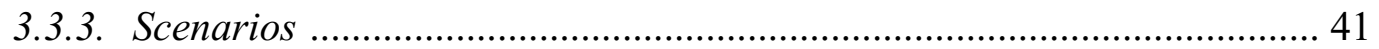

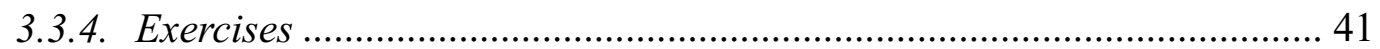

3.3.5. “As-is Scenario Map” Steps and Details .......................................... 42

3.3.6. “To-be Scenario Map” Steps and Details ........................................... 42

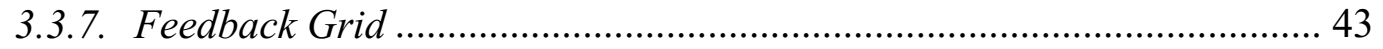

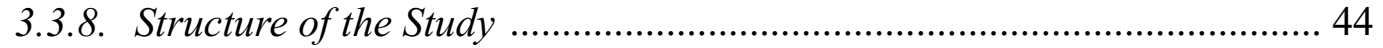

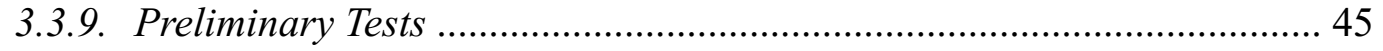

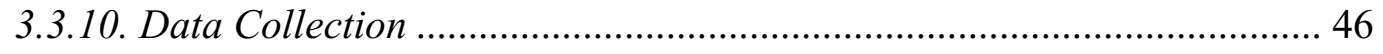

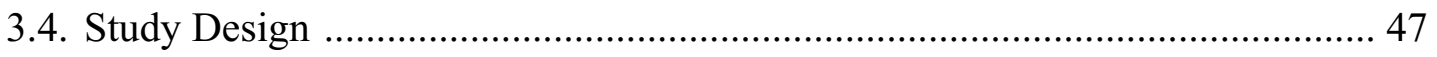

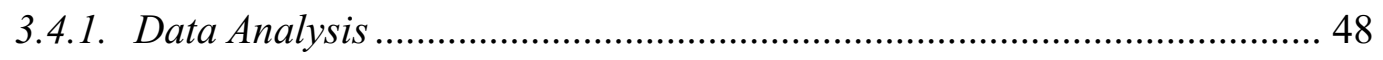

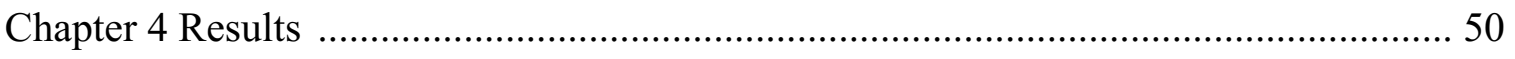

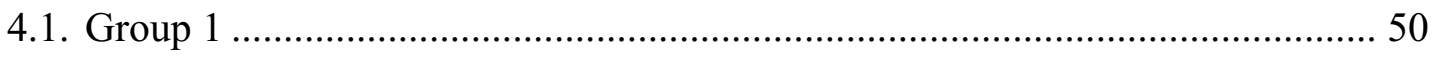

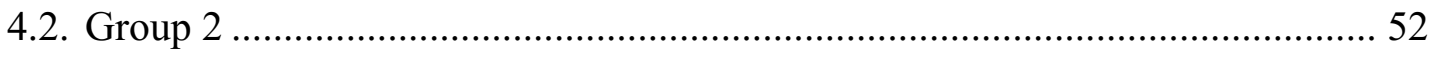

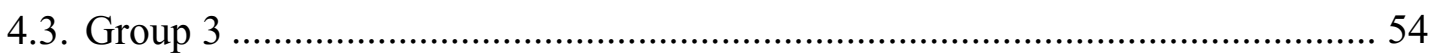

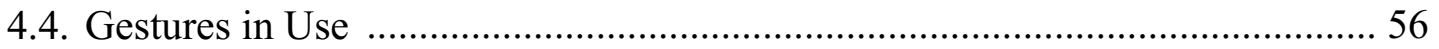

4.4.1. Gestures Participants Used, and Unique Interactions .......................... 56

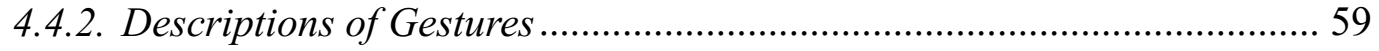

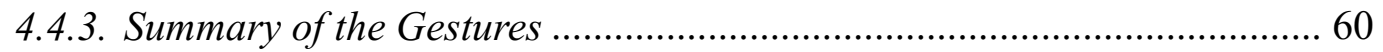

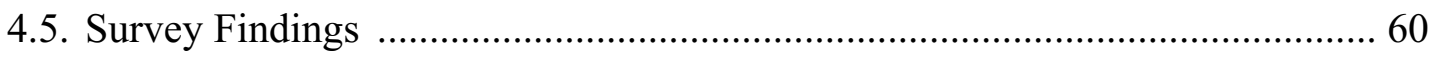

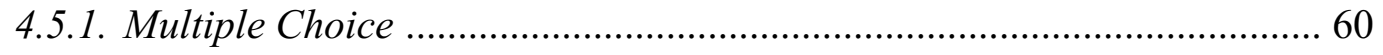

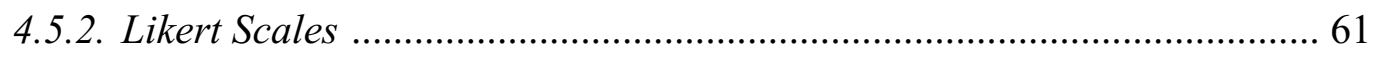

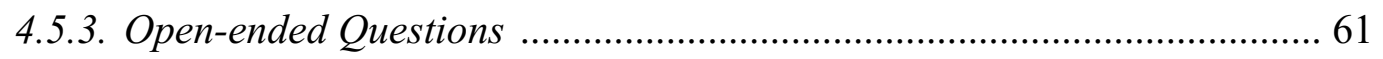

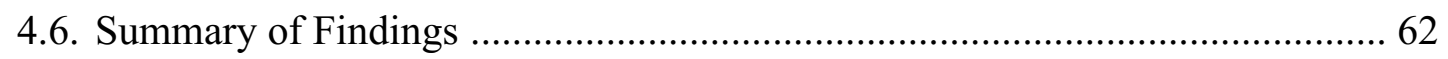

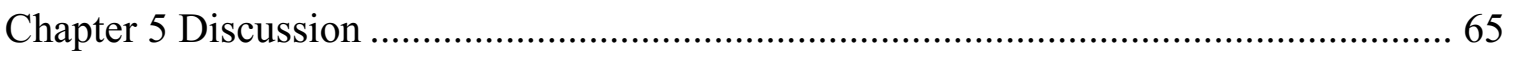

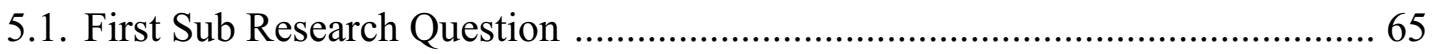




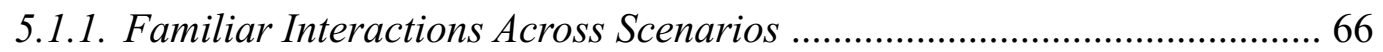

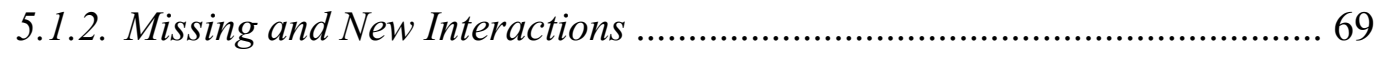

5.2. Second Sub Research Question ............................................................... 70

5.2.1. Tools used Across Media ........................................................................ 71

5.2.2. Interactions with the Media .............................................................. 72

5.2.3. Interactions with Other Participants .................................................. 73

5.2.4. Interactions with both Media and Participants .................................... 74

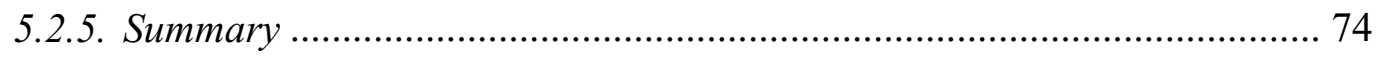

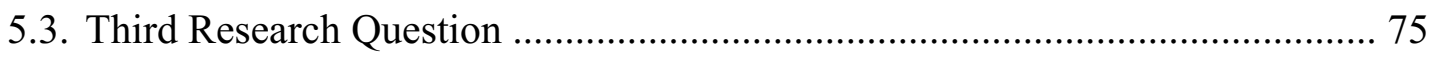

5.3.1. Feedback Mechanisms used in the Study ............................................ 75

5.3.2. Insights from Participants 'Actions .................................................... 77

5.3.3. What's Missing in Digital and VR .................................................... 78

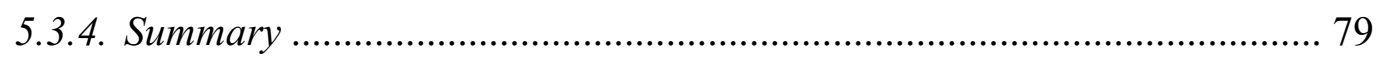

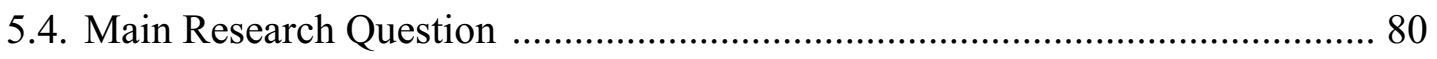

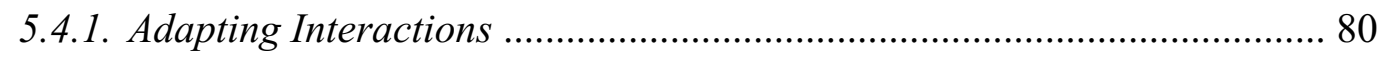

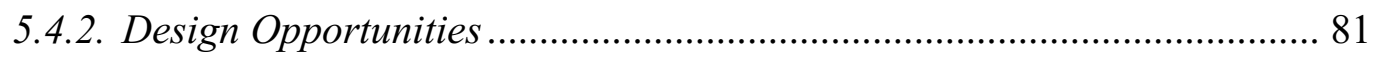

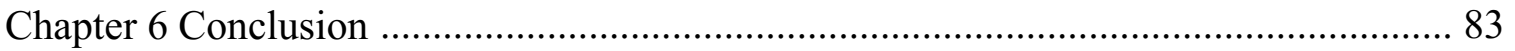

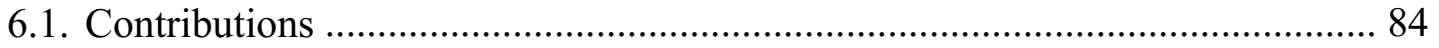

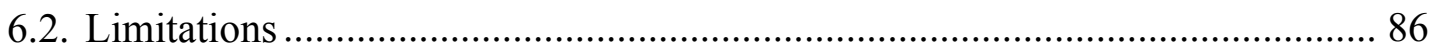

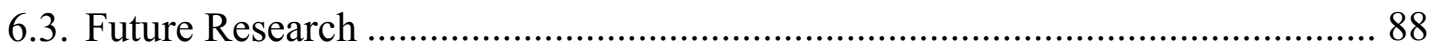

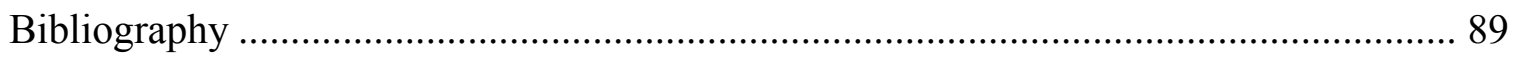

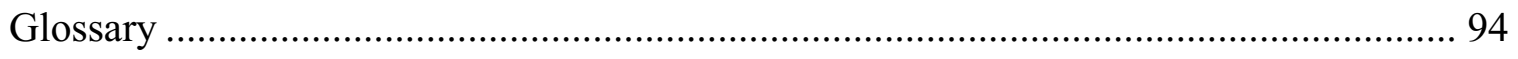

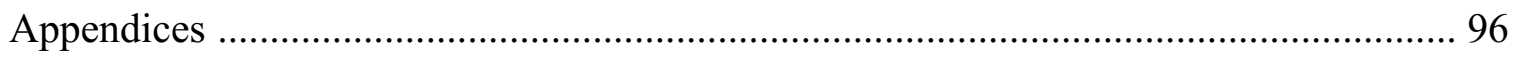




\section{List of Tables}

Table $\quad$ Page

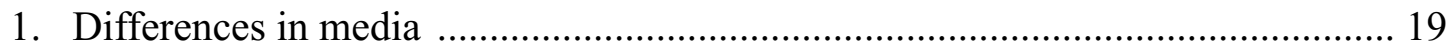

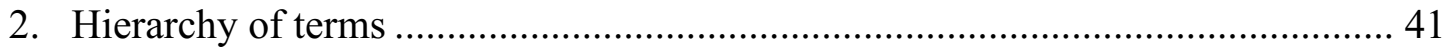

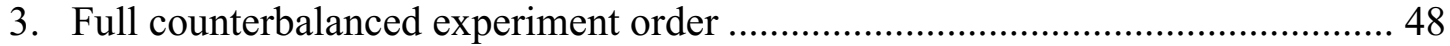

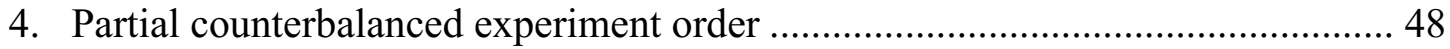

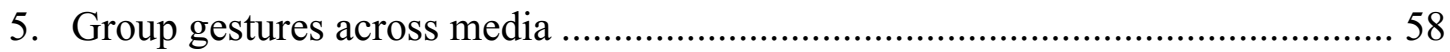

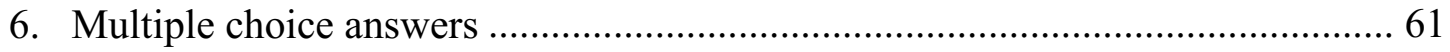

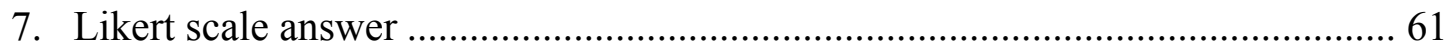

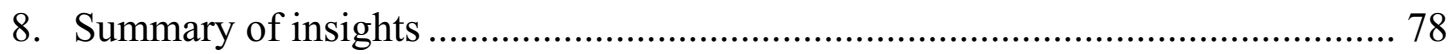




\section{List of Figures}

Figure $\quad$ Page

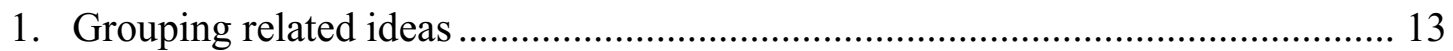

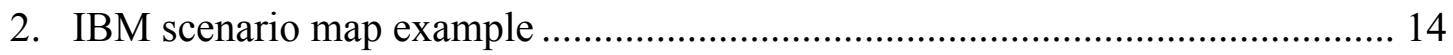

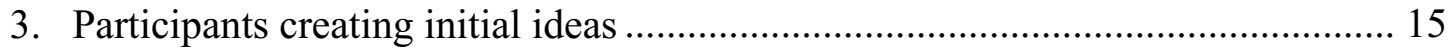

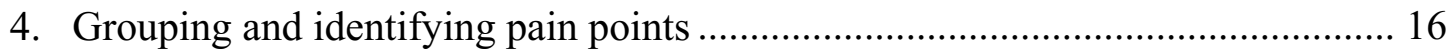

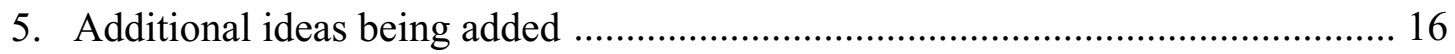

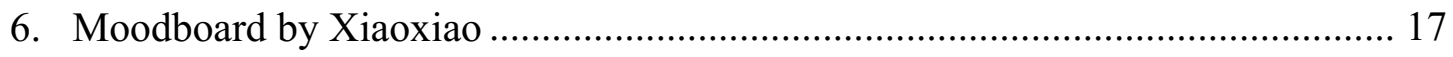

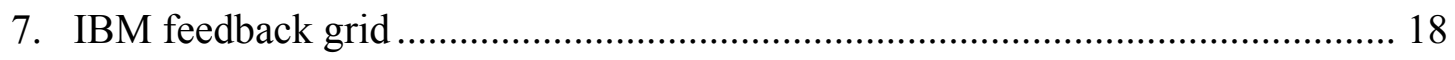

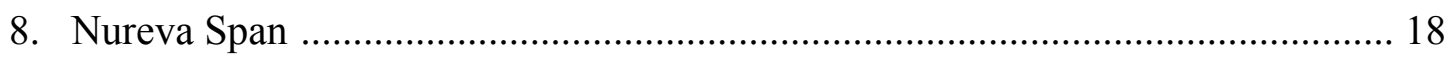

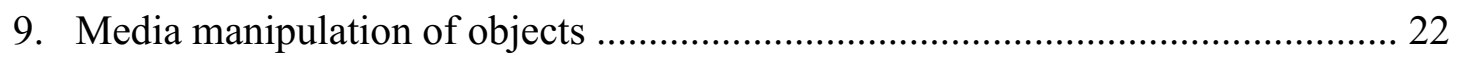

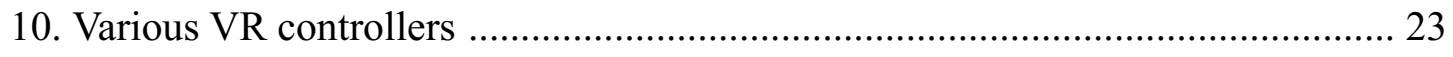

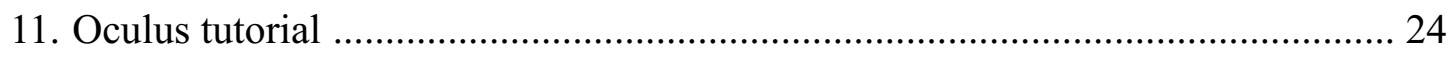

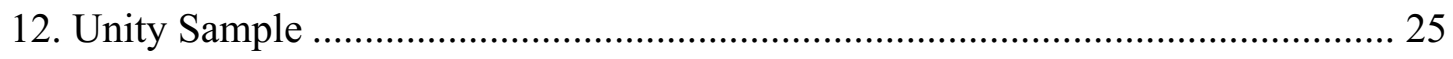

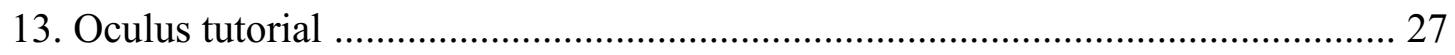

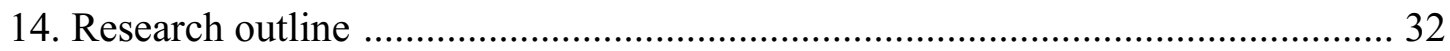

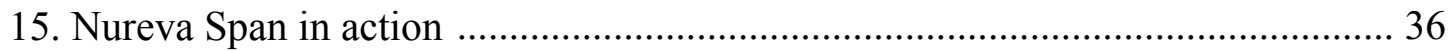

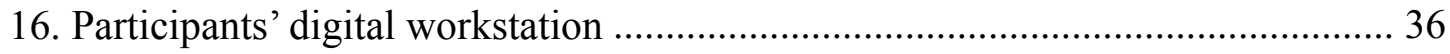

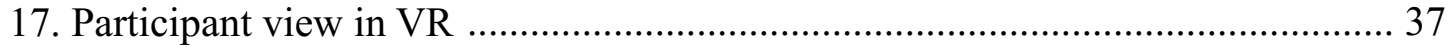

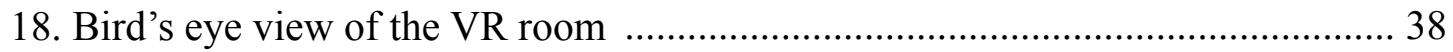

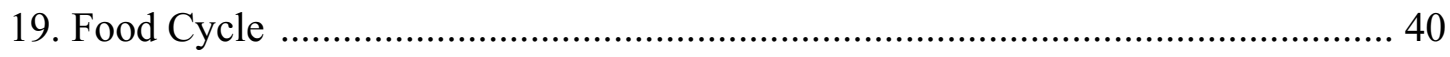

20. A finished as-is scenario map from the study ................................................. 42

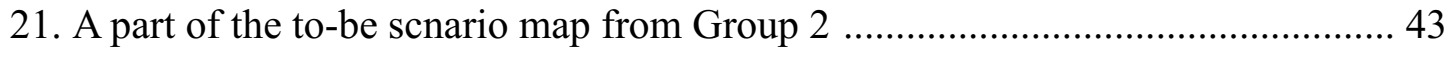

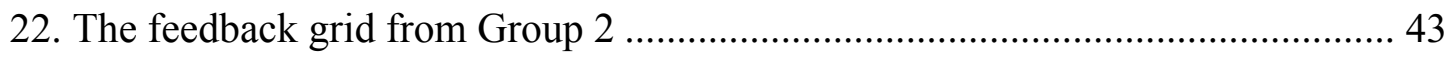

23. The three media the participants participated in ............................................. 44

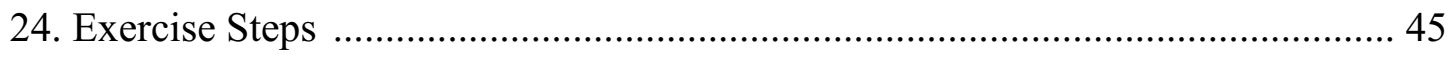

25. Activity graph for Group 1 by time and scenario ........................................ 51 
Figure

26. Activity graph for Group 1 by code and activity ........................................... 52

27. Activity graph for Group 2 by time and scenario ............................................. 53

28. Activity graph for Group 2 by code and activity ........................................... 54

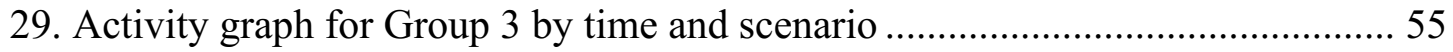

30. Activity graph for Group 3 by code and activity ........................................... 56

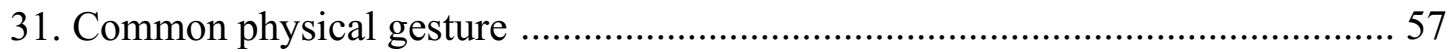

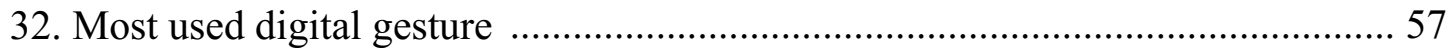

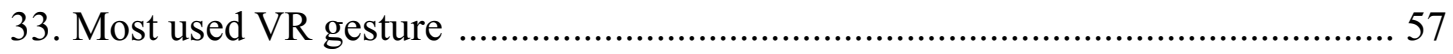

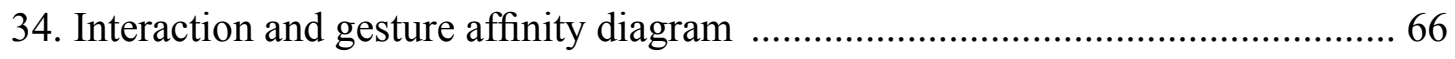

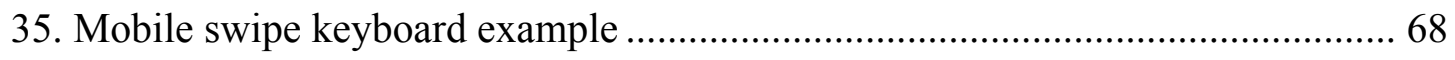

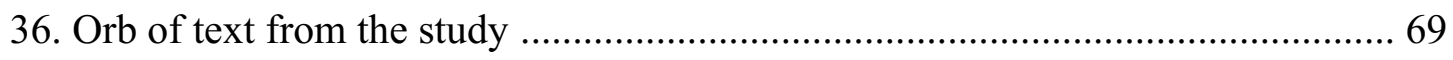

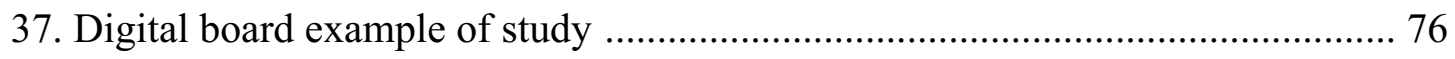

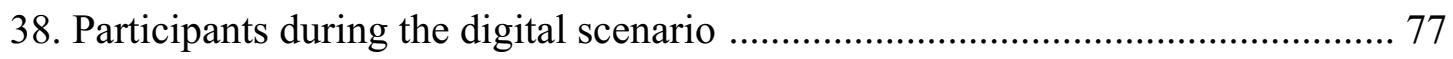




\section{List of Appendicies}

Appendix Name




\section{Chapter 1. Introduction}

Digital design tools are evolving at an increasing rate, as the field is constantly progressing. This constant evolution can lead to improvements in the way designers collaborate as well as record and archive their ideas. With virtual environments becoming more accessible to consumers and design teams, how would designers interact in this, asyet undefined (in a design context) collaborative space?

The intention of this research was to discover the differences between designers who perform design thinking strategies using different media and identify features that could be applied to VR thereby enhancing the user experience. The media that was explored included the use of brainstorming with a physical whiteboard, a digital whiteboard and a 3D whiteboard that was accessed using head mounted displays (HMD). The research observed gestures and interactions that were performed by the participants within all three (above) media and compared the interactions per minute to see where a method had elements that could be translated to the design of VR elements. The goal was to identify the nature of the interactive activities across methods and to create a set of recommendations that could improve the features of collaborative VR tools..

Interactions in the context of this research refer to any time a participant performed an action to create or modify content or had a meaningful exchange with other participants. Although the experimental study consists of different media throughout the exercise, the main focus of the research was to identify features that support design thinking collaboration and would be suitable for VR applications.

From this, the questions that thesis plans to answer are:

What are the interactions for collaboration and engagement among designers that are common in face-to-face experiences as well as 2D and 3D Virtual Environments?

This was broken down into secondary questions that aim to explore different aspects of the main question: 
1. What are the interactions that designers in design thinking sessions use in these environments now?

2. What are the tools for reproducing face-to-face interactions that are used now in digital and/or virtual environments?

3. Are there preferred visual, auditory \& haptic feedback mechanisms to enhance collaboration?

\subsection{Background}

There are many methods and techniques that designers use to develop ideas and quickly generate concepts. One of the most common is the use of brainstorming in face-to-face interactions using post-it notes, as seen in the image below (Figure 1), and often referred to as Design Thinking - "a methodology that imbues the full spectrum of innovation activities with a human-centered design ethos” (Brown, 2008). In physical meetings post-it notes provide the perfect medium for brainstorming (Brown, 2009; David Lavenda, 2014).

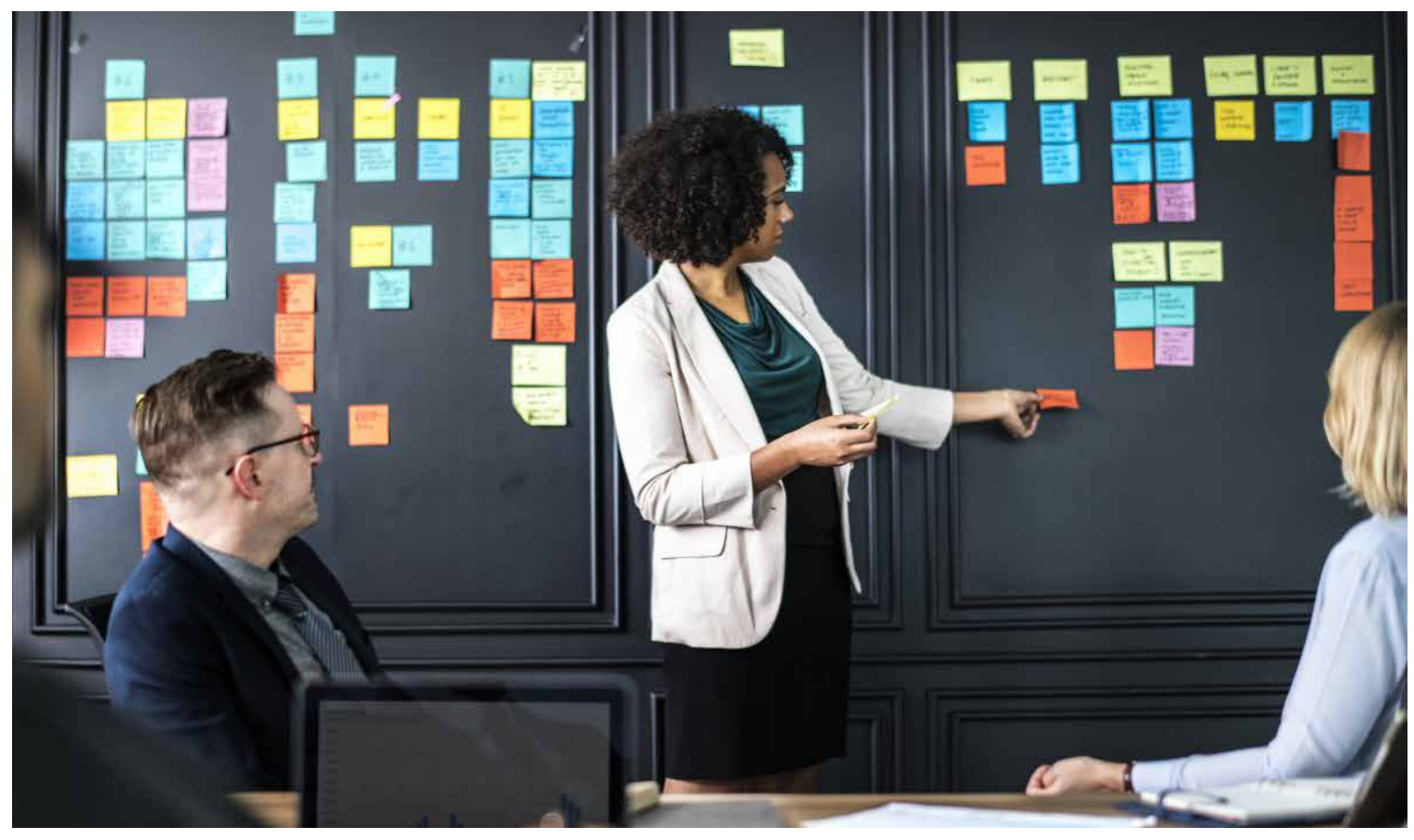

Figure 1: Grouping related ideas. Participants grouping related ideas together to help form a solution (Pxhere, 2019). 
Technology has also extended this exercise beyond the physical realm, as digital forms of the post-it-note exist through software like Nureva's Span system (Nureva, 2018) and Mural's online platform (Mural, 2018). This study considers how this practice could be experienced in 3D. It explores which features and interactions should be supported in a collaborative 3D virtual environment, based on common features used when collaborating in physical and 2D environments.

An example of a design thinking collaborative method is the Scenario Map, from IBM's Design Thinking Field Guide, seen in Figure 2 (IBM, 2017c). In IBM's design thinking process a Scenario Map is used by participants to figure out what is known about users in a given experience before they explore new ideas. The steps in this activity involve brainstorming everything that the participants think or know the users go through to complete a step in their overall activity. The example IBM uses involves each participant writing one idea per note on a post-it note; the notes are then placed on a whiteboard grid under different categories such as: preparing, planning, travelling and arriving at a destination, as illustrated in Figure 2. By the end of the scenario map and clustering or grouping together related ideas, collaborators would orally summarize their findings and figure out if any of the steps have problems, questions or possible solutions.

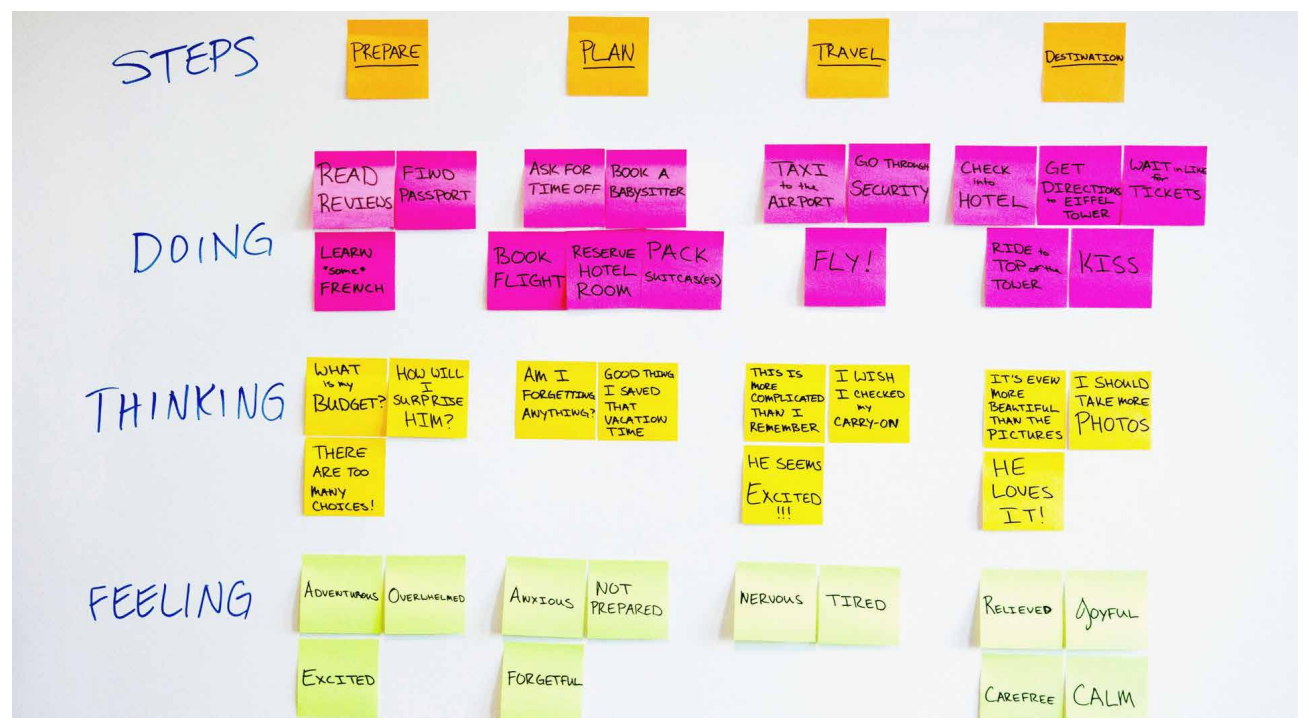

Figure 2: IBM scenario map example. (IBM, 2017a) 
Another example of a face-to-face interaction would be a group of designers who want to improve the user experience of ordering and receiving take-out food. They could start off by brainstorming their own experiences, and as a group, organize the notes as close as possible to their real experience categories. Part of that process is finding painpoints (problems in the user experience) and listing them where appropriate. Once most of the initial information is noted, the post-it notes with ideas on them are grouped by similarity and the group starts brainstorming solutions on additional post-it notes. After the group has enough solutions posted and grouped, the most important pain-points are identified for design improvement. The theoretical new user experience is then mapped out to visualize where and possibly how it might be improved.

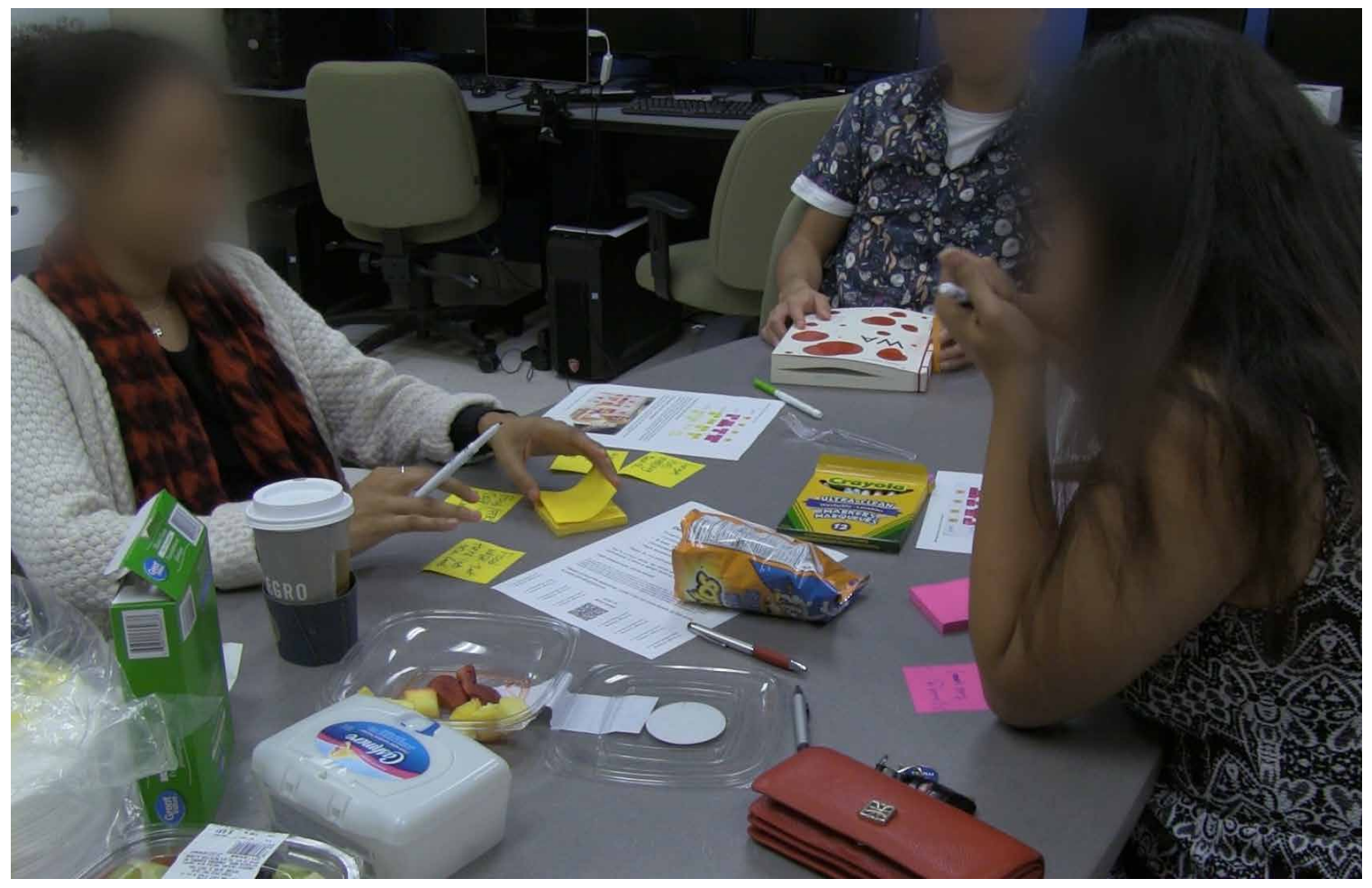

Figure 3: Participants creating initial ideas. Participants from the study completing the steps in the physical medium of face-to-face brainstorming to illustrate and clarify the above. 


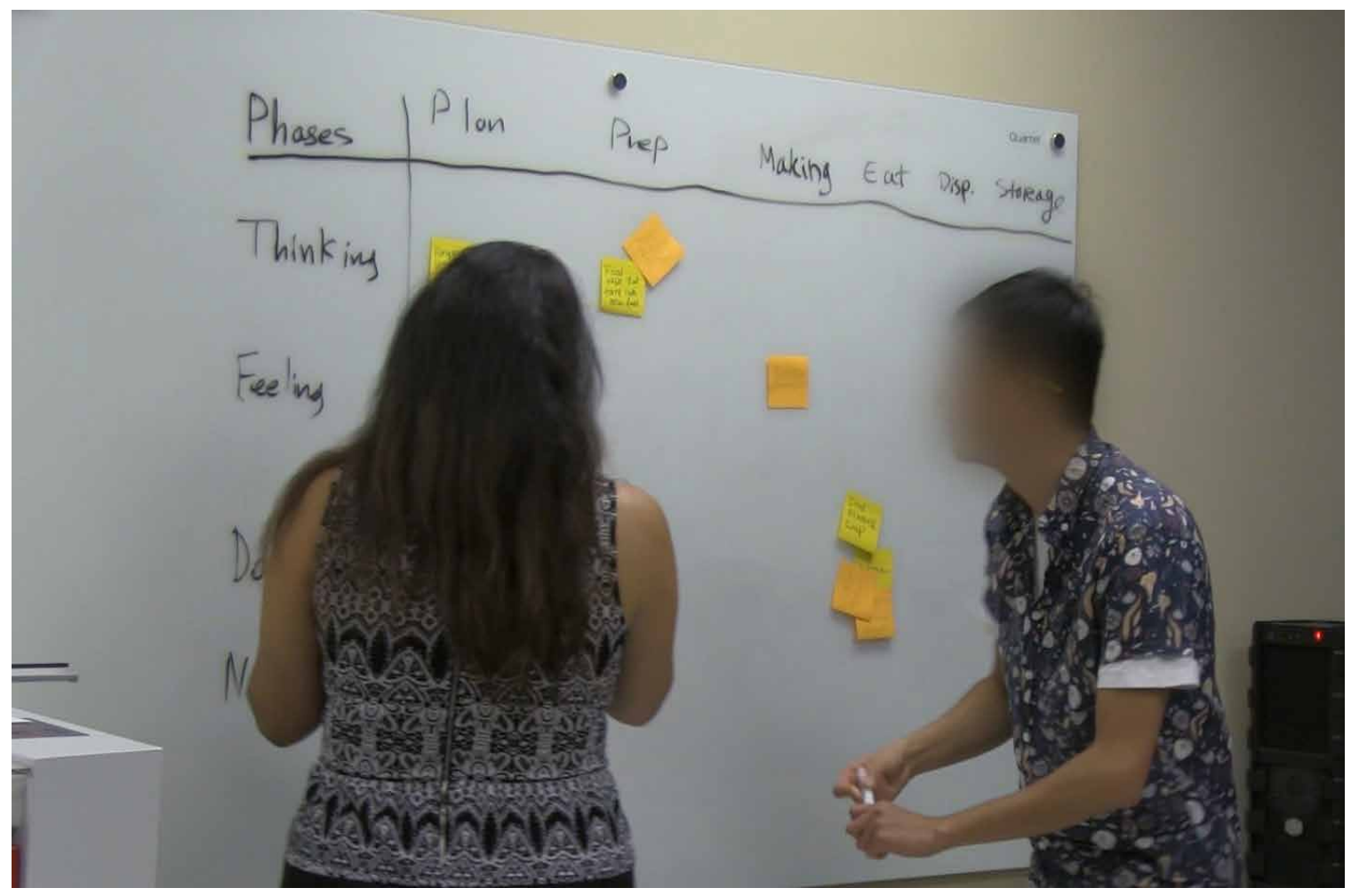

Figure 4: Grouping and identifying pain points.

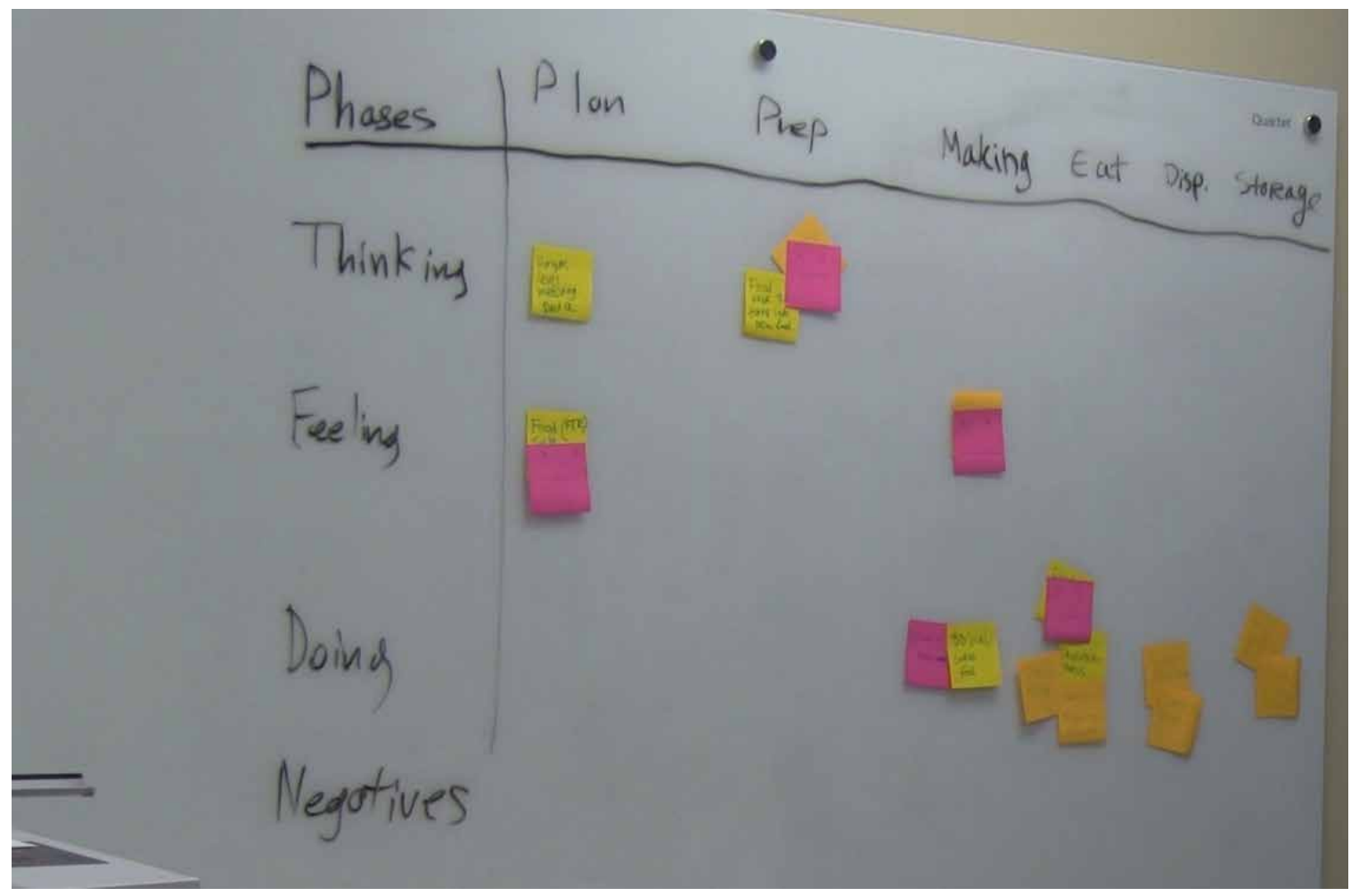

Figure 5: Additional ideas are added. 


\subsection{Inspiration}

Currently, some design teams use 2D online tools, such as Nureva's Span system, that support methods like moodboarding and feedback grids that are used in groups remotely to brainstorm and ideate. Moodboarding brings together a collage of images, text, and samples of objects into a single composition. In physical brainstorming media, moodboards (Figure 6) and feedback grids (Figure 7) take more time and resources to implement, for example taking the time to find and print digital content to put up on walls. An online tool like Nureva's Span and Mural includes functionality to do this without taking away valuable time.

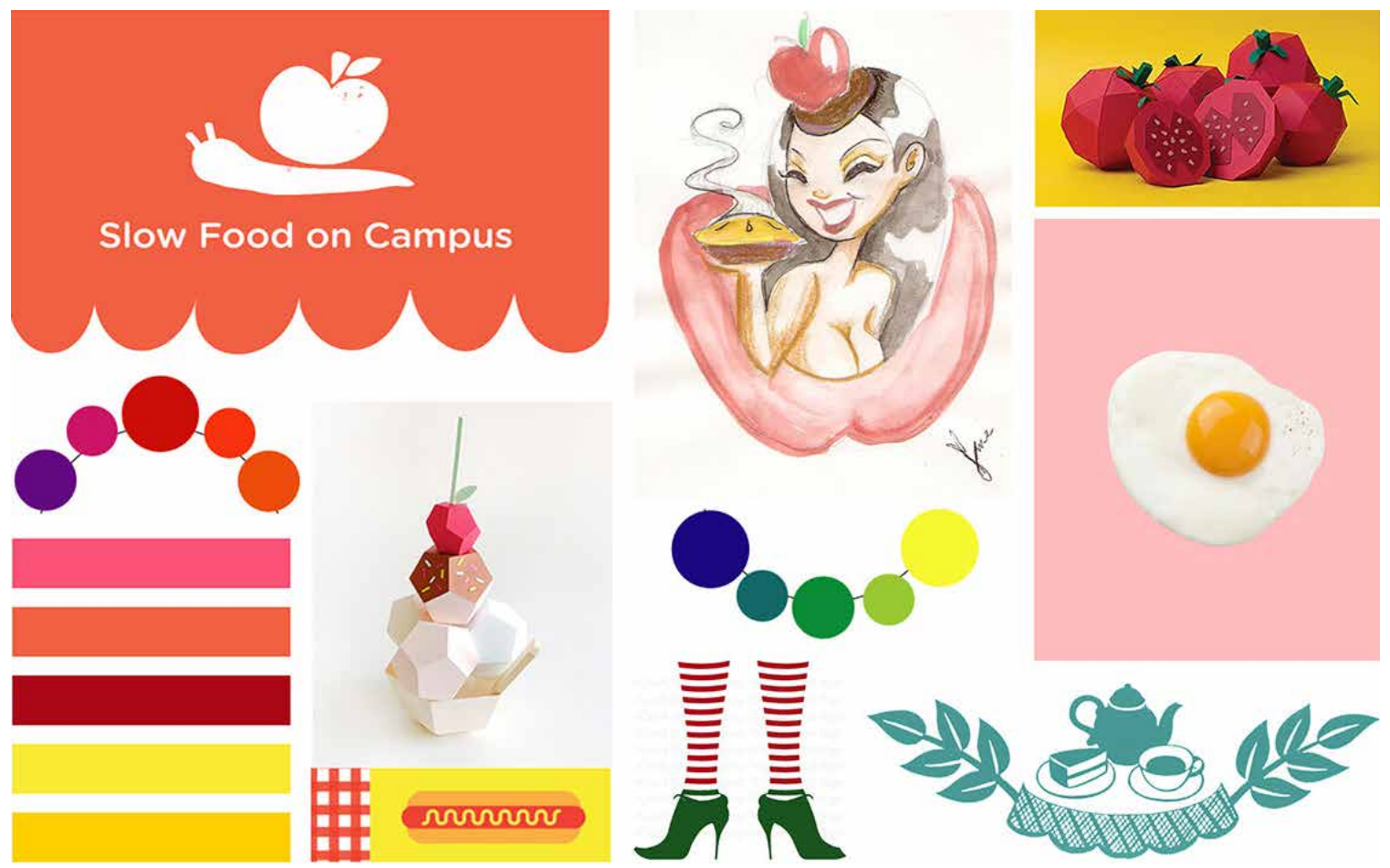

Figure 6: Moodboard by Xiaoxiao (Ovsyannykov, 2016). Showing different colours and inspiring motifs for a new project. 


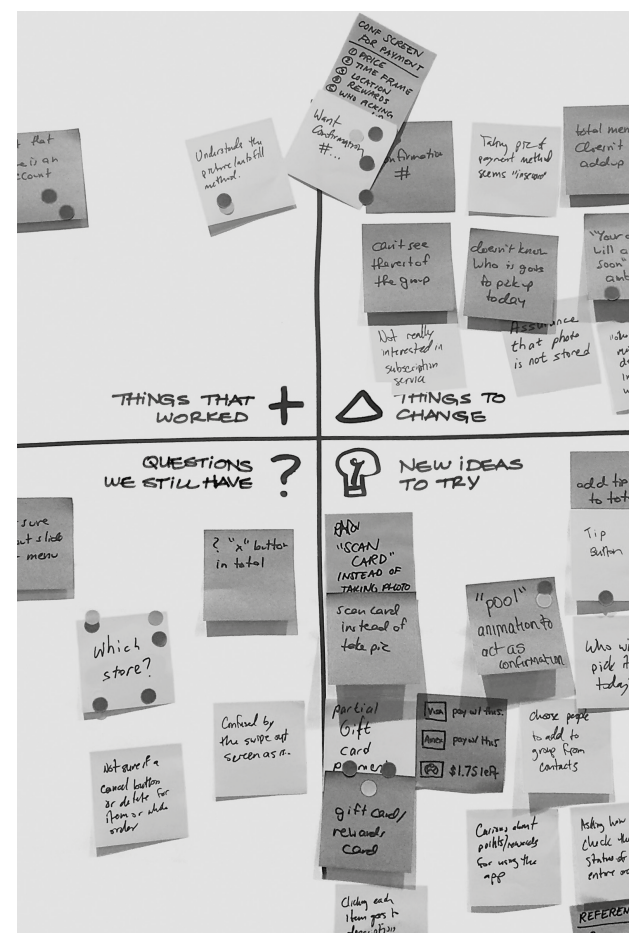

Figure 7: A feedback grid showing the four quadrants that make it valuable (IBM, 2017a).

Essentially, the teams set up virtual whiteboards with software counterparts of post-it notes, images and sketches. One benefit of digital adaptations is that they allow for quick search and placement of images used in moodboards (Figure 8).

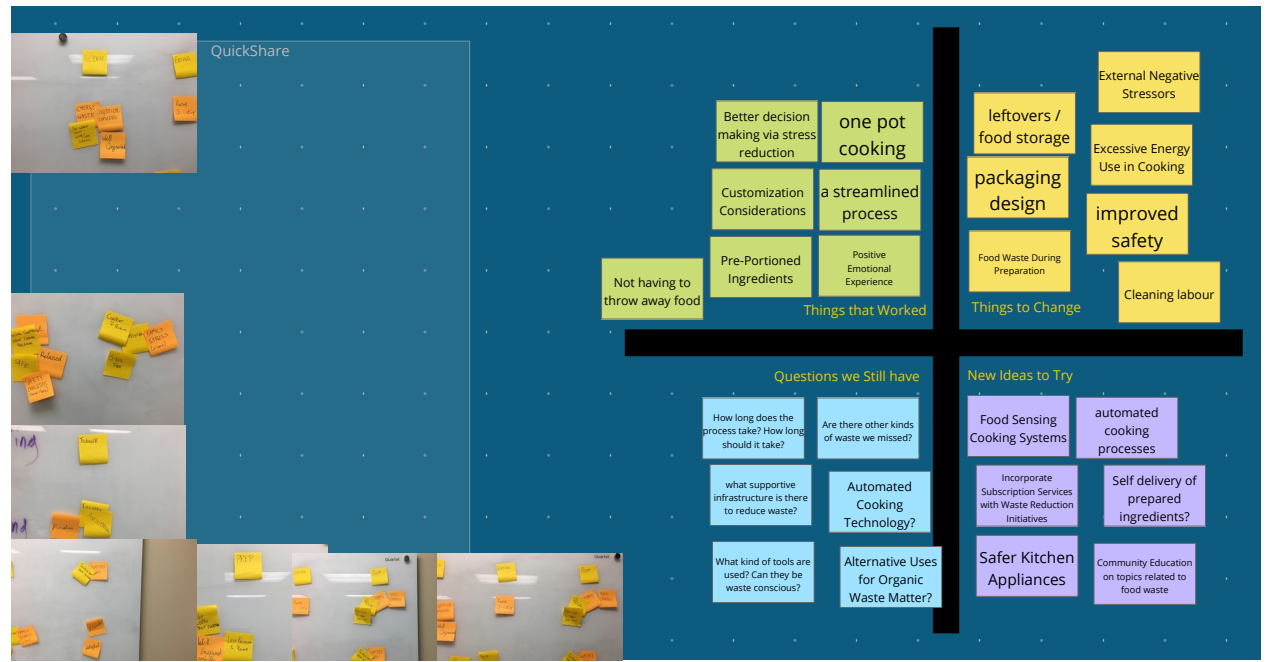

Figure 8: Nureva's Span. A screen shot from Nureva's Span system showing notes for Group 3 and images from the previous scenario they completed. 


\begin{tabular}{l|l|l|l} 
& $\begin{array}{l}\text { Physical } \\
\text { Post-it Notes }\end{array}$ & $\begin{array}{l}\text { 2D: Mural } \\
\text { or Nureva Span }\end{array}$ & 3D: VR \\
\hline Text & Yes & Yes & Yes \\
\hline Indexed for Search & No & Yes & Yes \\
\hline Media (photo/video) & Only Printed & Yes & Yes \\
\hline $\begin{array}{l}\text { Person to Person } \\
\text { Interaction }\end{array}$ & Yes & No & Yes \\
\hline Remote Participants & No & Yes & Yes
\end{tabular}

Table 1: Differences in media.

Physical brainstorming (face-to-face) offers relatively little interactivity as shown in Table 1 when it comes to collaborating with others located remotely and makes it harder to use other media such as images. 2D media like Mural or Nureva's Span offer much more in terms of allowing remote collaborators to easily join and contribute, as well as providing the flexibility to add images and links. 3D media like VR may take the digital space further by supporting the presence of remote collaborators and closely mimicking the physical media while also allowing the flexibility of a digital medium (David Lavenda, 2014; Swing, 2000).

\subsection{Purpose of the Research}

The purpose of this research was to observe the interactions that occur in faceto-face as well as 2D and 3D Virtual Environments - for collaboration and engagement among designers (i.e. navigation, selection, manipulation, text input, among others). The main objective of this study was to develop recommendations for VR design thinking tools that are based on common features observed in the three design thinking media mentioned above. A second objective was to identify appropriate visual, auditory and haptic feedback mechanisms that seem to enhance collaborative interactions in all of the media. 


\subsection{Contribution}

The findings from this research may contribute to new knowledge and recommendations when designing other multi-user VR studies and programs for design thinking. This may provide insight into environmental and sensory aspects of feedback that also play a key role in giving users the proper cues to keep them engaged and active.

\subsection{Structure of the Thesis}

Chapter 1 introduces the background and inspiration of the study and illustrates examples of design thinking. It describes the purpose of the study and objectives of the research, along with the contributions.

The second chapter is the literature review that provides a context for this study and acknowledges the work of other researchers in different disciplines. At the same time the literature review identifies aspects of work that provides the foundation for this study. Topics range from observing the user experience, protocols for collaborating, communicating, nature of the environment or context, to social interaction. This interdisciplinary approach looked at topics in fields such as industrial design, humancomputer interaction (HCI), interaction design and behavioural psychology. A history of the use of virtual environments is included. The rationale for the study is then presented, with questions and subquestions built from the literature discussion. The methods appropriate for further research are mentioned briefly and expanded upon in the third chapter.

The third chapter opens with a short explanation of the choice of a repeated measures method of research. The main information in this chapter is the detailed and step-by-step description of the methods, users, location, tools, ethics protocol, and methods of analysis.

Chapter four presents the findings from the research methods by participant group and method. It starts by describing the results and the compiled analysis by interaction. Then it goes into the demographics and participant preferences to illustrate how this has affected the types of gestures and interactions used during the study. 
Chapter five is a discussion based on was observed and the analysis and synthesis of the data. This chapter synthesizes the data across groups and scenarios presented to the participants. It concludes with insights that may be suitable for design thinking features in VR. Chapter six contains a summary of the data and talks about the limitations as well as next steps. It focuses on the findings, contributions and possible opportunities for future research. 


\section{Chapter 2. Literature Review}

This chapter presents a review of academic and published literature that explores a range of perspectives such as: new technologies in the VR space; the underlying intricacies of user interaction in collaborative spaces; recent research from an industrial design perspective of user experience and behaviour in $2 \mathrm{D}$ vs $3 \mathrm{D}$; social interaction from psychology; protocols for collaborating, communication, and the nature of context from $\mathrm{HCI}$.

\subsection{How Behaviours Differ in 2D vs 3D}

This literature review looks at what is required to perform the interactions of navigating, gesturing at, selection and manipulation of objects, sensory input and feedback, speed, and grip. It studies the literature that explains how they differ in faceto-face, 2D and 3D interactions (Figure 9). Figure 9 is organized into three columns, and each column depicts the different features of each medium. The chart shows that each medium involves different interactions to achieve similar results.

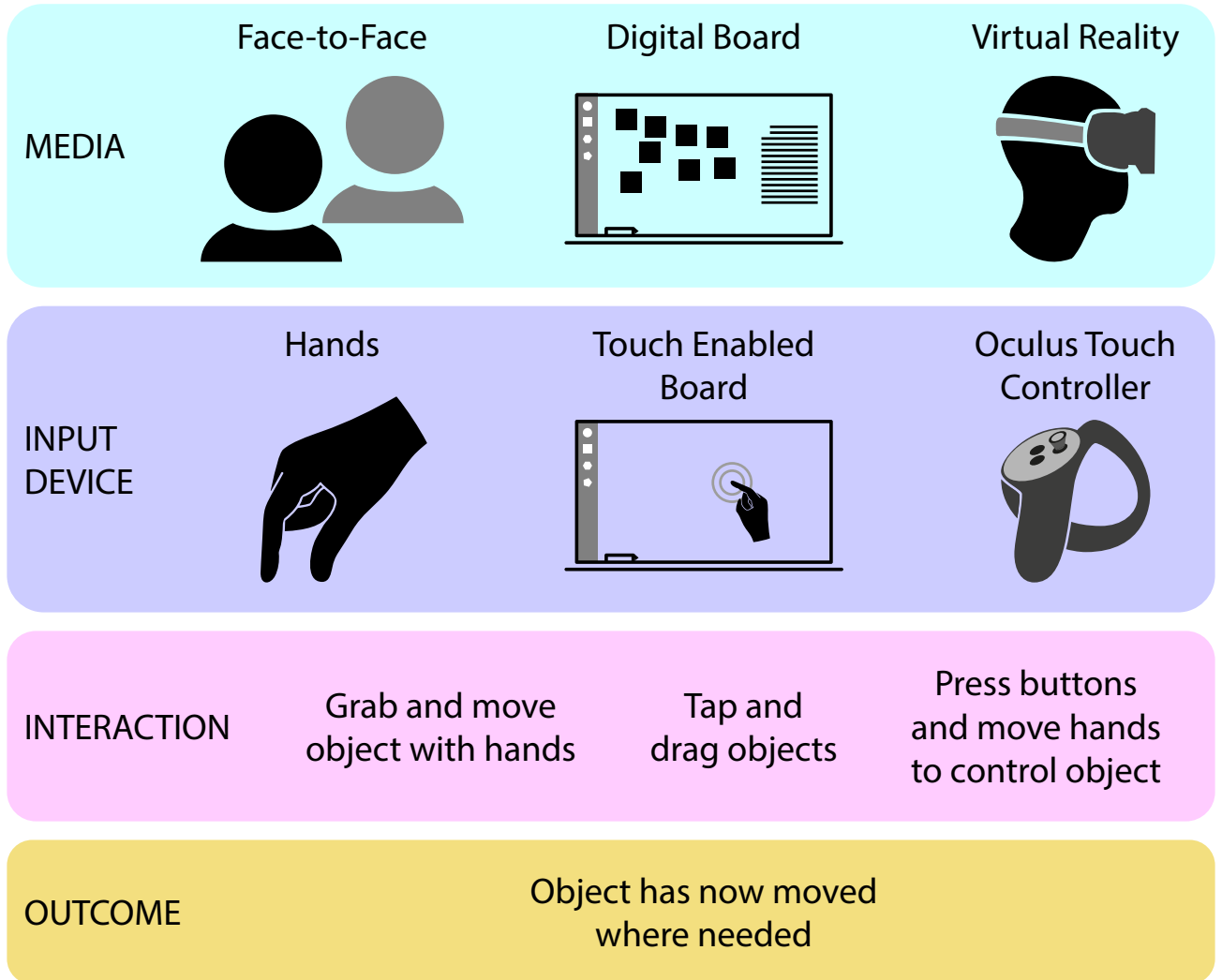

Figure 9: Media manipulation of objects. 
Objects, like the post-it notes in real-time design thinking practices, are the most common interactive elements in a 3D space. While earlier iterations of 3D environments focused on allowing users to experience and explore a virtual environment, a way to manipulate the objects within the environment was still needed. The problem, however, was that there was no knowledge about how to design such complex 3D interactions in a way that was usable and effective (LaViola Jr., 2017). The act of manipulating an object in an isolated space as a single user is much easier to understand and replicate than a group of users all trying to act on one object at the same time (Heldal, Spante, \& Connell, 2006). Lloyd et al., (1999) describe how multiple users can interact with objects that appear within the virtual environment. Their experiment used the herding of virtual creatures as the object of interest. Although it was just an introduction to the method they used, they conclude that an application programming interface (API) could allow designers to more easily create and manipulate formations (arrangements of objects).
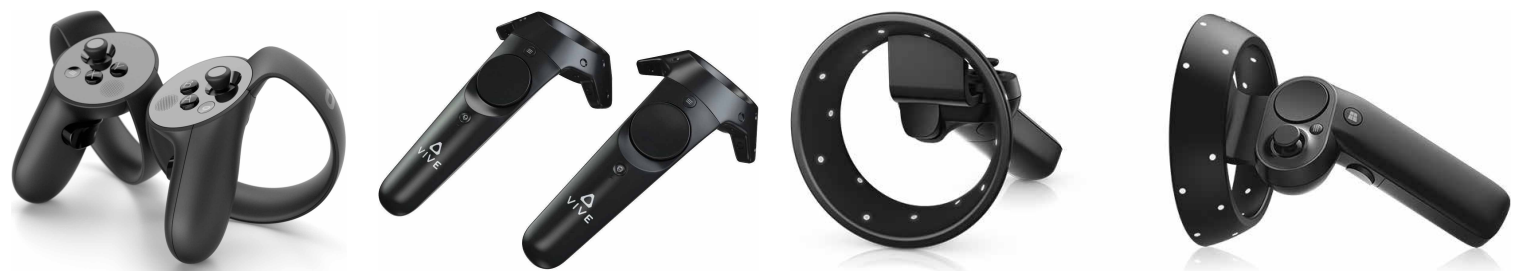

Figure 10: Various VR controllers. From left to right - Oculus touch, HTC Vive, Dell Visor controller (“Dell Visor," 2018; “HTC Vive Controller," 2018;

Oculus, 2018).

There is currently no standard design for VE input devices, however there are many devices made specifically for interaction in VR such as: The Oculus Touch controller (Oculus, 2018), Dell Visor controllers (“Dell Visor," 2018) and HTC Vive controllers ("HTC Vive Controller," 2018). They all employ 6 degrees-of-freedom (DOF) absolute tracking which provides the 3D position of the controller in space. DOF is the number of simultaneous axes of translation and rotation supported. A 6 DOF device supports movement in the $\mathrm{x}$ - (left/right), $\mathrm{y}$ - (up/down), and z-axes (forward, 
backwards) as well as rotation about these same axes. Other controllers for consoles and computer gaming have also been rigged to work with VR experiences such as a PS4 or Xbox controller. Although these controllers could be used for physical input, the digital representation often uses ray casting (Figure 11) and virtual hands ( $\tilde{\mathrm{A}} \&$ Argelaguet, 2007; Ro et al., 2017). Direct manipulation for 3D interfaces should be the most intuitive and efficient method of interaction because it would act just like reality, but it also has problems such as fatigue and the physical equipment required to receive input (LaViola Jr., 2017). Apart from the interface itself, latency is problem we still face as lag between input and output can cause cybersickness due to the mismatch between the visual and vestibular systems. The only requirement for an interaction device in $3 \mathrm{D}$ space would be to match the equivalent DOF or map an appropriate device such as a mouse to 6-axis type of devices.

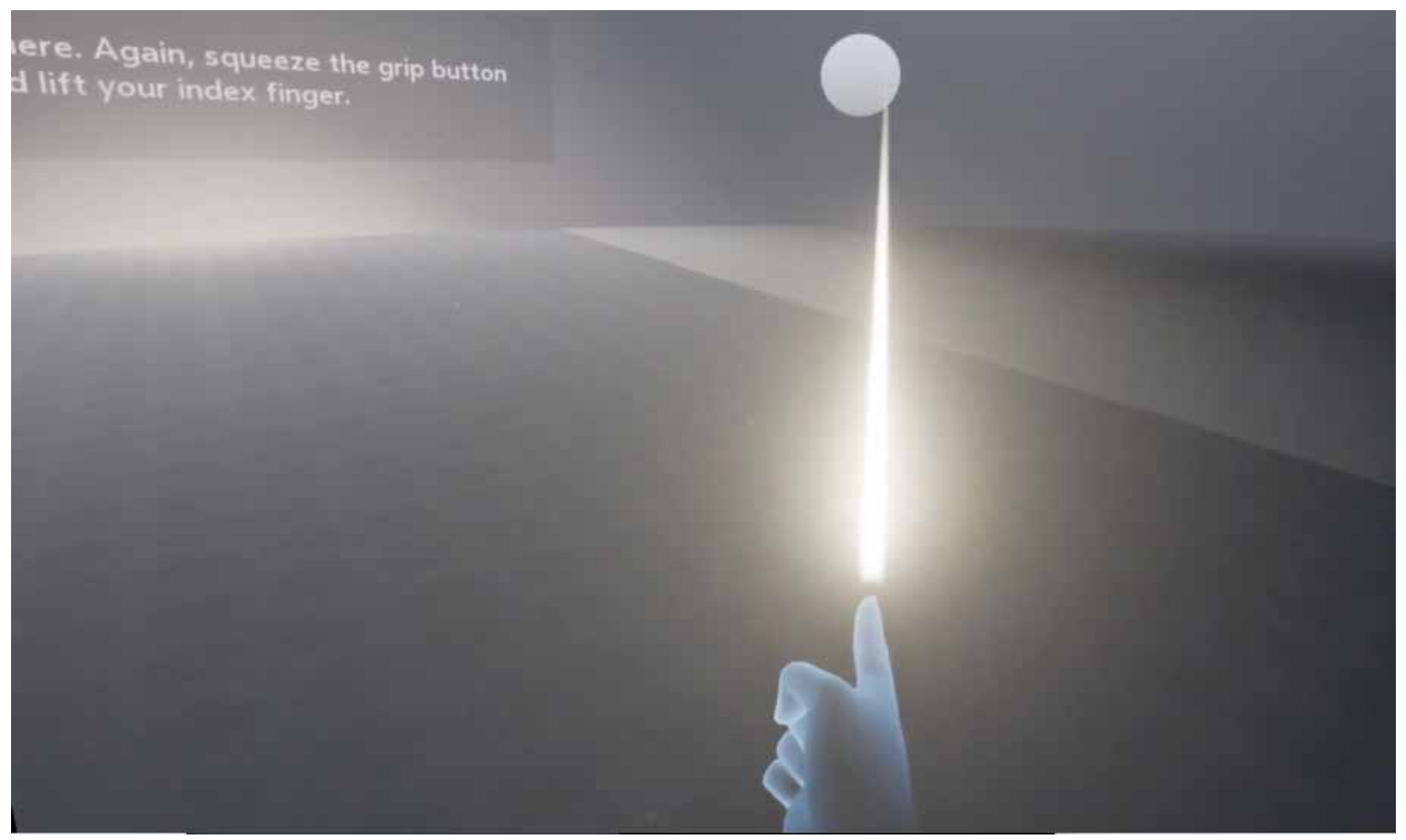

Figure 11: The Oculus Rift tutorial showing a virtual representation of the user's hands using ray casting to point at an object (Oculus, 2018). 
Patel et al., (2006) explore some of the reasoning behind developing a standard or guideline for interaction methods to control what happens in these environments. They note that the physical input device is not the only part that could use a standard interaction. New context menus are needed in 3D environments as well, to better suit the experience and flow. With a better flow the behavioural difference in $2 \mathrm{D}$ versus $3 \mathrm{D}$ can start to blur and become one in the same. An example is system control (LaViola Jr., 2017) for the VR menus that most applications already use (Unity, 2018).

Currently, developers use flat or curved panels (Figure 12) with selections as if the viewport was a series of monitors. Simple 2D context menus work in most situations, but lose the benefit of the third dimension according to Patel et al. (2006). An example of a 3D menu could be changing options by interacting with the topology of a virtual 2D panel. Menus in 3D space need to be easy to navigate and logical, to make decisions quickly, just like the $2 \mathrm{D}$ counterparts. Linear menus may be the easiest to successfully implement by taking advantage of linear mental models that people already have with computers (Bae et al., 2012; Patel et al., 2006).

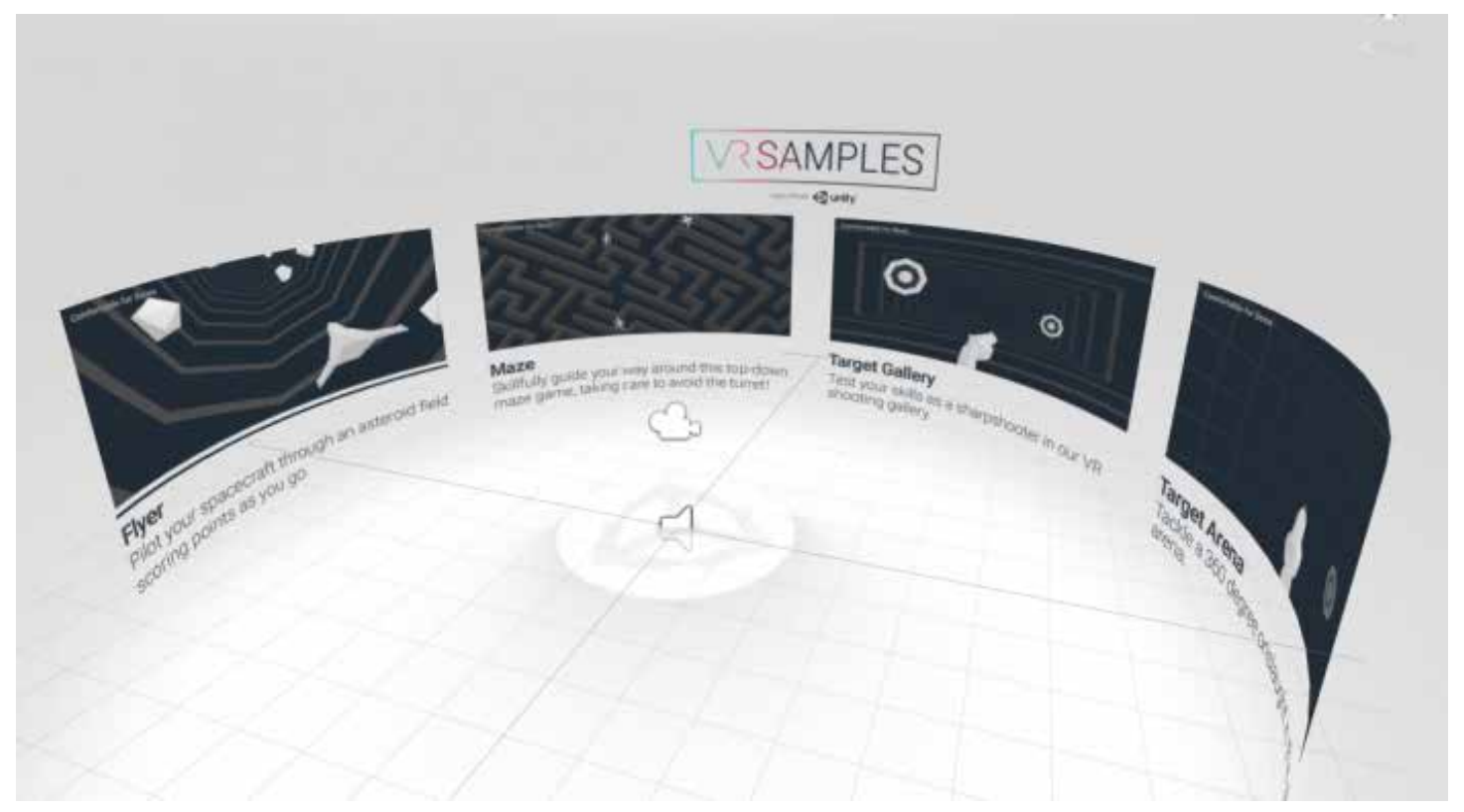

Figure 12: Unity Sample showing how some menus in VR mimic 2D displays (Unity, 2018). 
Sensory information is a valuable asset in every aspect of product design (Spence \& Gallace, 2011) as it can help inform users of what the norm or protocol is between participants. Mason (2001) talks about how to integrate sensory information into a virtual environment by looking at what is required to make it feel natural. Users in a collaborative or co-located environment who can manipulate objects and artefacts need a way to communicate feedback about these manipulations (movement, modification, relocation, resizing) to all the other users to help prevent miscommunication. Mason's research focuses on testing how users interact with objects and each other using variables such as speed, grasping and other forces associated with handing other users the virtual objects. She is planning to use this data for improving the design of user interface systems to provide effective feedback to users.

\subsection{Protocols for Collaborating}

Different visual cues in a mixed reality environment with group awareness are tested in Salimian, et al. (2016). Using virtual, augmented and mixed reality they wanted to understand whether being immersed in a dynamic workspace enables local and co-located collaborators to have equal and richer interactions than what is currently achievable in a 2D space. They were looking for an opportunity to combat the effect of remote people having less of an impact on discussions and sometimes feeling left out. The authors also explored how people respond to visualizing the movement of arms and limbs in the virtual environment. Cues that are used in a virtual collaborative space must clearly define what the actions of each cue will cause, given that most people do not have mental models or cultural norms for interaction in 3D space. For example, recent VR experiences like the tutorial for the Oculus (Oculus, 2018) show touch controllers illustrating the user's hands (Figure 13), but not all gestures are second nature when interacting with the controllers such as individual finger movement. Users tend to pick up familiar gestures that they have been performing for years such as swipes and flicks, used on smartphones, more readily. 


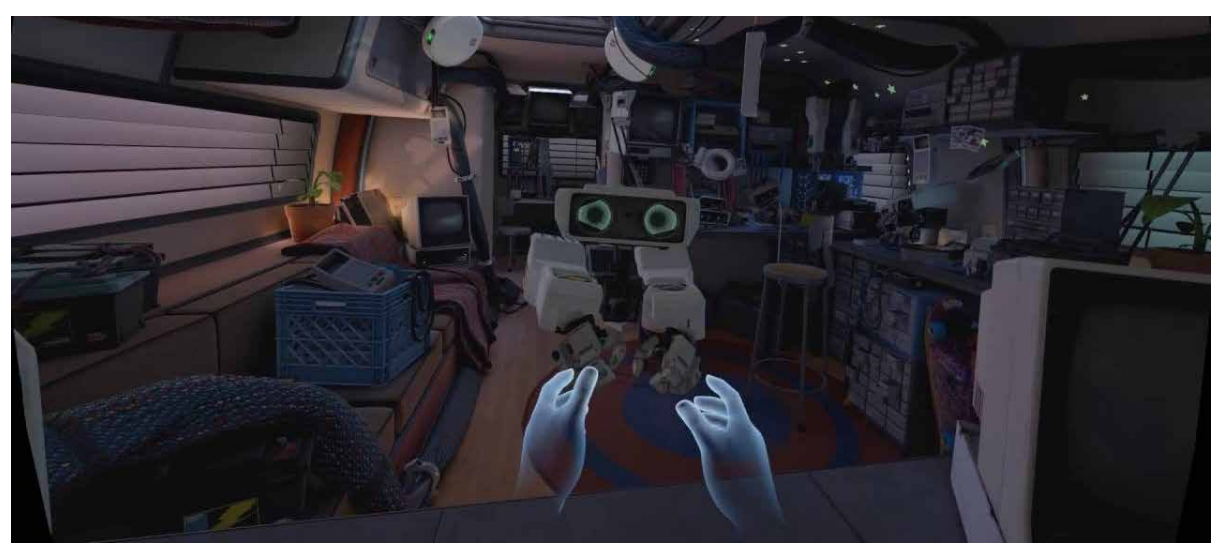

Figure 13: Oculus Tutorial (Oculus, 2018). Showing virtual hands as representations of the controllers in $3 D$ space.

Musse, Babski, Capin, \& Thalmann, (1998) explore group behaviours in a virtual system with emphasis on groups. They examine people's patterns - how they act with others in a group and how systems can emulate real world experiences. They found that keeping information between systems separate and transferring them to a main server gave the best results by limiting interference from other users in the system. The problem of integrating real movements and slight intricacies is not an easy task for a virtual system (Mason, 2001; M. H. Salimian, 2015).

In the case of remote collaborators, Chellali, Milleville-Pennel, \& Dumas, (2008) found that collaborators need to have a common frame of reference. This can be achieved by providing replacements for different sensory modalities, such as a certain object or the origin of a sound that remains in the same place for everyone. The example they used was the location and orientation of tetraminos based on spatial representations and descriptions, either relative to the user or the environment from the user's perspective. For many of the users they tested, landmarks within the environment remained the same for all users and made the identification of objects of interest relative to each other for the experiment. They found that men were more easily than women able to use the environment to understand the objects within it by referencing the landmarks. Women tended to use more personal relations to understand and describe an object. This may 
be important when it comes to interactions between collaborators and objects as certain gestures that use references could be easier for some and harder for others and how men and women differently understand objects will need to be taken into consideration.

\subsection{Communicating}

Communication, where the user and system do not speak the same language, for this study would be considered an interpretation task by the user interface, (LaViola Jr., 2017). This means that the system interface has to be fluent in a language that the user understands and can be easily interpreted by the system. This is important for the study because some of the participants may be learning how to use certain tools for the first time, such as VR. If the participants are able to easily interpret what both the system and other users are doing, communication may be much smoother. Navigation through menus and communication within virtual systems is a complex problem, either using special controls to allow for full 3D movement or locking controls into 2D planes within a virtual environment. Whatever is used needs to be intuitive for what the user wants to do, nothing should be lost in translation. Gauglitz, Nuernberger, Turk, \& Höllerer (2014) focus on how drawings from a 2D perspective should translate to a virtual environment. In some cases, such as tutorials or demonstrations, being able to draw and point inside a virtual environment is a very important aspect for the transfer of information. They also looked at what user-interface controls could translate most easily, specifically with a touch-enabled interface. They found that gestures already in use by smart phones and tablets were easy to translate with very minimal assistance. Some of the more complex gestures that users might not be familiar with were not as easy to translate, but were still understood by the participants, such as orbiting a scene. When compounded by multiple users trying to communicate with each other, the complex gestures become even more important.

Miller (2010) focuses on the idea that intercultural interaction and collaboration could be improved using virtual worlds as a meeting ground. Using virtual games as the medium, this researcher has found that in some cases, collaboration leads to success and 
group steps are completed easily. In other cases, especially when cultural differences are vast, the team appears to do worse and communication breaks down. Part of this is due to the barriers that exist from the participants' home country and culture.

\subsection{The Nature of the Environment or Context}

Learning collaborative methods with computers has some barriers to entry that have made it difficult for participants to make the direct transition from real experiences to digital and virtual. Mainly due to the time required to learn software, costs associated with software and the initial hardware that may be required for setup. While methods without the use of a computer are easy to understand and learn quickly because of learned mental models, a computer-assisted version may require a different way of looking at the problem that leads to an unnecessary learning curve (Tiwari \& Holtham, 1998). Not only that, but the affordances, intuition and mapping are much clearer in reality than in GUIs. The transition to computer-assisted collaboration may also lead to better archiving of past sessions that make up for the barrier to entry as a requirement to be able to review past work.

While a collaborative environment should be relaxed and open to ideas for discussion, the learning phase in computer-supported environments can cause a bit of stress for the first few minutes (Swing, 2000). A warm-up period may allow everyone to get comfortable without the pressure of getting something done. Being able to communicate who is doing what is a feature that is easily noticed in physical face-to-face interactions, but in computer-supported environments it becomes a much more complex problem that requires both programming and user-experience design. Turn taking may be able to solve the problem, but that relies on the group agreeing to take turns and may limit spur of the moment creativity and exploration. It may also require that the participants are aware that if more than one person tries to activate an object at the same time, unintended effects may occur. 


\subsection{Observing the User Experience}

Task analysis is a structured method of looking at one step of a multi-stepped activity and understanding the components that it takes to accomplish it (Kuniavsky, 2003). It can be used to find out how people deal with a particular workflow and find ways to get all the steps done. For example, searching for a keyword may be an important part of locating similar points in a digital medium. The steps involved in this activity can be broken down through observation and are based on the participants performing gestures across the different tasks. Some of these interactions may be similar across the activity while others are the differentiators between the steps.

Interaction design is reliant on the participant, as the product itself does not exist in a rational and substantial way until the context is observed (Kolko, 2011); it relies on interactions between the participant and the product for the design to become fully realized. From the participants' perspective, it is important for them to interpret available tools, while the researcher would seek to understand why they would use one over the other. When asked why the participants do the things they do, interpretation and analysis is always needed to figure out what makes a design valuable or not. This can lead a designer to better empathize with the target users (Kolko, 2011).

Being able to see firsthand how the body language and interactions change between the methods may also provide clues as to how engaged the participants are in the activity. Kolko (2014) confirms this when he notes that "Behavioural signals are also a part of this and observing is simply the process of being there as it happens". According to Kalbach (2016), mapping experiences in the study makes it much easier to compare between media when the participants are asked to show how they might do a step. This combination of observing and inquiring about what the participants feel may expose why one medium is better than another for collaboration. 


\subsection{Framing the Research}

From this literature review, it seems that while attempts have been made to create a collaborative environment in different media, they do not always convert well from 2D to 3D. Many attempts leave much to be desired given the missing interactions and functions that should be augmented when moved to another medium (Heldal et al., 2006; LaViola Jr., 2017; Patel et al., 2006). This includes input methods that have become inair gesture based and extends to the user interface that is not always intuitive (Bianchi, 2015) and sometimes requires a different UI between platforms. This also aligns with how communication between participants changes when moving from a physical face-toface to a digital representation (M. H. Salimian, 2015). Capturing subtle movements (e.g., shrug of shoulder, glance, yawn (Schwartz et al., 1998)) and body language (e.g., folded arms, smiling, fiddling (Kuhnke, 2012)) is a big task for consumer-grade VR devices to handle, much less properly communicate it to other participants in a meaningful way.

The last issue with getting a VE to be collaborative is setting up the right context and environment that encourages interaction and creativity (Tiwari \& Holtham, 1998). Ironically, learning to operate the VR system creates barriers if the UI is unintuitive and requires a warm-up/onboarding period that slows the whole process down since the whole point of VR systems is to leverage real-world ability.

This interdisciplinary approach involved the disciplines of design and computer science, especially information technology in HCI. The contributions from each for the study was the use of design thinking methods from industrial design, study design and technology from HCI. The design thinking methods used in this study were important because of the collaborative interactions that were observed needed the participants to work together during problem solving. Process and identifying the interactions were significant from HCI due to the complex devices needed to conduct the study. While the knowledge from the literature and experience from supervisors also benefitted from their background in $\mathrm{HCI}$. 
Based on the insights from the literature review the main research question is:

What are the interactions for collaboration and engagement among designers that are common in face-to-face experiences as well as 2D and 3D Virtual Environments?

This was broken down into secondary questions that aim to explore different aspects of the main question:

1. What are the interactions that designers in design thinking sessions use in these environments now?

2. What are the tools for reproducing face-to-face interactions that are used now in digital and/or virtual environments?

3. Are there preferred visual, auditory \& haptic feedback mechanisms to enhance collaboration?

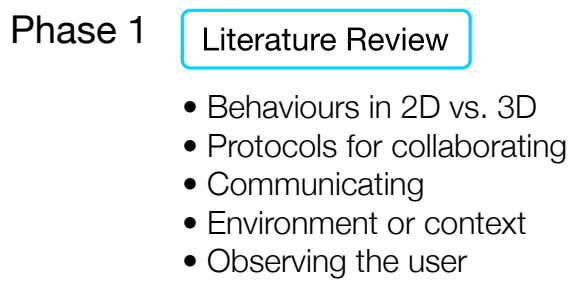

\section{Questions}

- Interactions that occur

- Tools in different media

- Feedback to enhance collaboration
Phase 2

\section{Ethics}
- Application
- Approval

\section{Methods}
- Study design
- Participant criteria
- Apparatus
Pilot Study
- Test equipment
- Finalize procedure

Phase 3

Recruitment

- Recruitment materials

- External contacts

- Scheduling
Data Collection

- Pre-test survey

- Observations

- Post-test survey

Phase 4

\section{Analysis}

- Observations into criteria

- Triangulation

- Visualization

\section{Conclusions}

- Insights

- Recommendations

- Limitations

Time

Figure 14: Research outline. 


\section{Chapter 3. Methods}

This research adapts a repeated measures design (Cozby \& Bates, 2015) approach to answer the main question presented in the previous chapter, "What are the interactions for collaboration and engagement among designers that are common in face-to-face experiences as well as 2D and 3D Virtual Environments?" It uses a mix of quantitative and qualitative open-ended inquiry to understand the users' perceptions related to their interaction and communication. This approach explores the experiences of the participants using the different media environments in relation to the quantitative nature of interactions involved in the tasks performed in the different media.

The method of repeated measure (Charness, Gneezy, \& Kuhn, 2012; Cozby \& Bates, 2015) is a method in which all the participants within a group experience each level of an independent variable, which in this case is the media type. A reason for using this include fewer participants needed to complete the study as the participants can serve as their own control group when they complete the physical brainstorming medium. A disadvantage to using this method is the possibility of practice, fatigue and contrast effects (Cozby \& Bates, 2015). Practice effects occur when participants perform the same task over and over, getting better each time. This would skew the number of interactions and performance if the media was presented in the same order each time. Fatigue effects would be participants getting tired or bored when doing the same or similar tasks again and again. Contrast effects are when independent variables effect each other when presented in succession. To counteract this, partial counterbalancing was used to assign the groups explained further in section 3.4.

Quantitative measures were used to collect data that would provide measurable and trackable information about participants' actions and behaviours. The use of qualitative measures provided data derived from the participants' perceptions which could be compared or triangulated (Martin \& Hanington, 2012) with the quantitative data obtained from observations. 
Therefore, this study approach brings together data collected from observing and quantifying participants' behaviours with their perspectives about collaborating in different interactive media environments. This took place in three different exercises as described below in section 3.3.3.

This process generates data that can be analysed separately and synthesized across the participation groups to see trends among different interaction codes. The process used for analysing this information was the triangulation of data gathered by the researcher from observing the scenarios, pre and post surveys to the participants, and activity intensity. The reason this analysis method was chosen is because it is useful for research involving qualitative and quantitative data like observations and surveys. Triangulation is a method of using different research tools to gather supportive data from many angles (Martin \& Hanington, 2012).

\subsection{Participants}

Due to the plan to use design thinking activities in the study, the participants would need to have design thinking experience. This would enable them to more easily adapt to the changes in use of media since they already have an understanding of the process. Through a contact at IBM Ottawa's design group, designers were recruited to participate in the study. This expanded the pool beyond Carleton University's student designers to include experienced professionals.

Therefore, participants were recruited through convenient purposive samples from among designers in the Ottawa area. A challenge with recruiting the participants from IBM was scheduling around their busy work. Initially several participants were interested, but in the end only three were able to make it. When it came to designers at Carleton, not many students were interested in the study. This led to a total of six students.

One group consisted of Carleton Master of Design students, one of undergrads in Industrial Design and the last group included employees from IBM. They were grouped like this instead of being mixed due to scheduling conflicts with work or school and time 
availability. These participants were chosen for their background with design and design thinking methods and experience with computers. No restrictions were placed on age or gender.

In total nine people participated across three groups. Two participants were women and seven were men. Participants were contacted via email invitation (Appendix E) outlining what the study was about and what they would be doing, after which interested volunteers reached out.

\subsection{Apparatus}

Each medium used different software, and in one case no software was used at all, to complete the steps. For the physical use of post-it notes, no software was needed as the participants were only using post-it notes and a whiteboard.

- Physical medium - a whiteboard was used as the medium with post-it notes and markers.

- Digital medium - a digital whiteboard was setup on a large television screen with several computers in front of it. Participants had access to post digital post-it notes and the ability to annotate them.

- VR medium - a private VRChat room is set up with the ability for the participants to write anywhere on the board.

\subsubsection{Software}

The digital medium used two applications on multiple computers. The host computer used Nureva's Span workspace software in combination with a proprietary touch-enabled projector (i.e. Figure 15) on a whiteboard to allow the participants to interact with the Span digital board called a canvas. This interactive canvas allowed the participants to touch and move objects on the Span digital board and annotate text/ drawings directly. Each participant optionally used a client computer running Google Chrome to send digital notes (Figure 16) to the host computer via the internet that they could then move around on the Span digital board. 


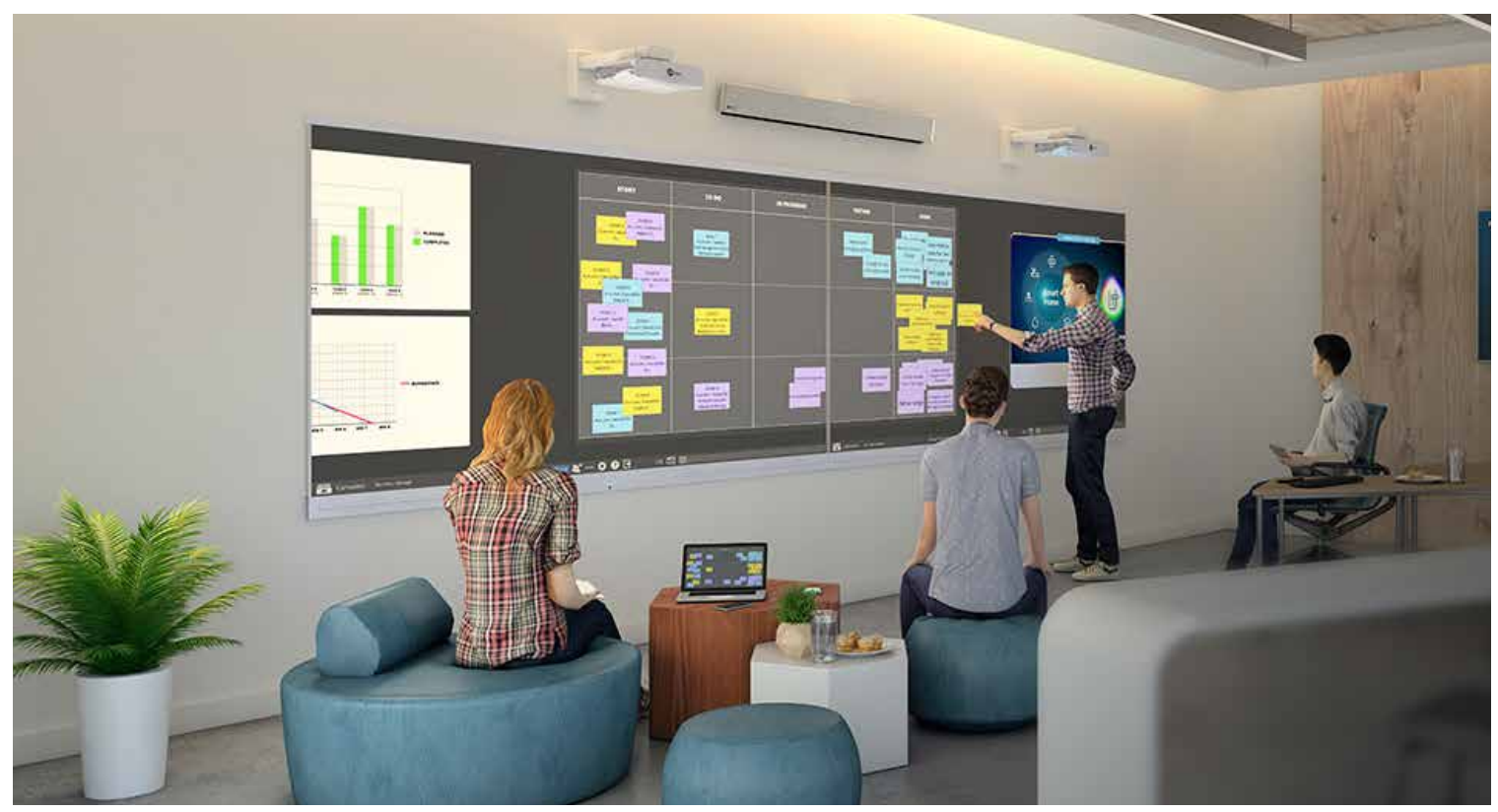

Figure 15: Nureva's Span in action. Promotional image from Nureva.com showing a user interacting with the wall to move a digital note in the Span software (Nureva, 2018).

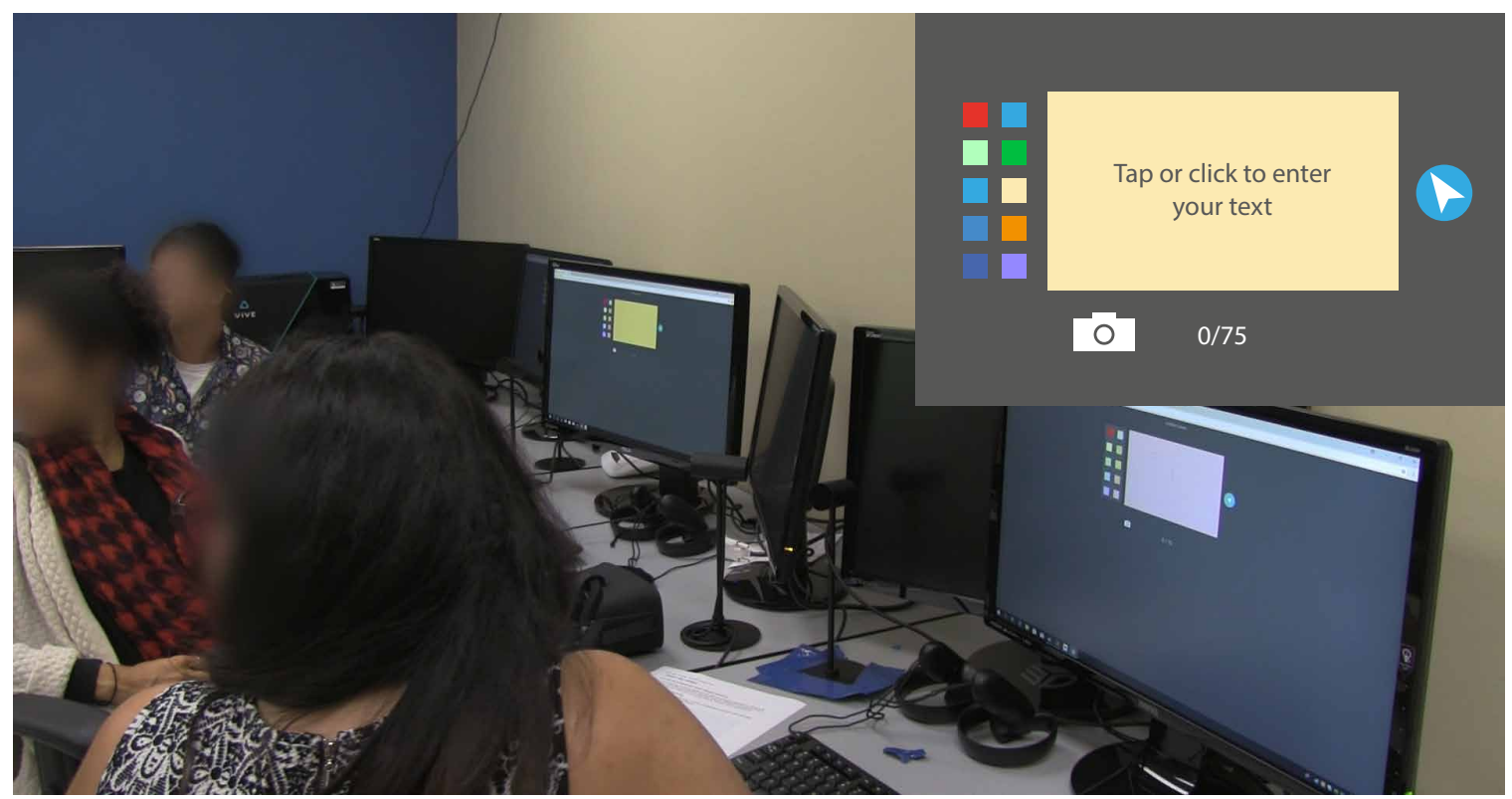

Figure 16: Participants' digital workstation. For typing up digital notes using Google Chrome. 
For the study, the program that had the most potential for use in VR was called VRChat. It is a game based on the steam gaming platform that allows users to choose different avatars and explore a world created by others and themselves. A premade environment existed in the game that could serve as the backend for the VR because it had the most tools available that would work with the study.

The VR medium had each of the participants at a different computer (see above), each equipped with an Oculus Rift, a head mounted display developed by Oculus VR (Oculus, 2018) that supports stereo vision and positional tracking. The software for this medium included Steam for the game VRChat (Figure 17) that was used to host the participants in VR and Nvidia GeForce Experience that was used for recording the gameplay. VRChat is a socializing game that has avatars that chosen by the users that move around in worlds that are either user-created or already exist in the software. The interactions that take place between the users usually consist of voice chat and body movements; experienced players are able to articulate the 3D models in more complex ways than a beginner would.

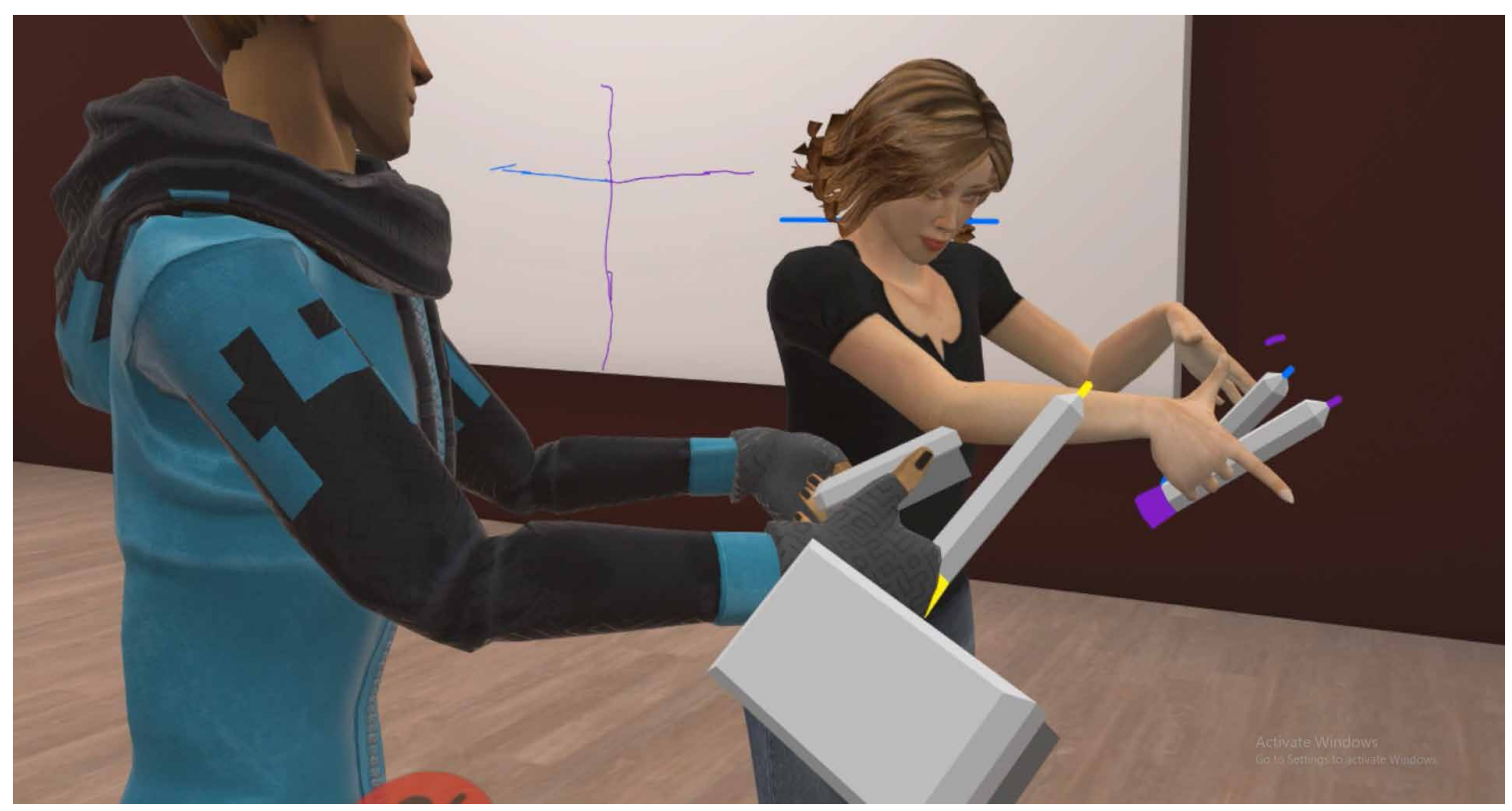

Figure 17: Participant view in VR. A participant view of the VR medium in VRChat, a VR game that allows a player to control an avatar's actions and movements. 
For this study, a world called the boardroom was used because it contained tools similar to the other media such as whiteboards and markers. A private link was setup for use by the participant computers only. The virtual room consisted of several whiteboards placed on all four sides with a stage in the centre (Figure 18). On the stage were three sets of markers and erasers for the participants to pick up and use. Participants could write in two modes: in-air and on-wall. When writing in-air, movement of the marker tip in 3D space left a marking that could be interpreted differently depending on the angle it was viewed from (i.e. if a participant decides to write while spinning around they would end up concealed in a cylinder of text).

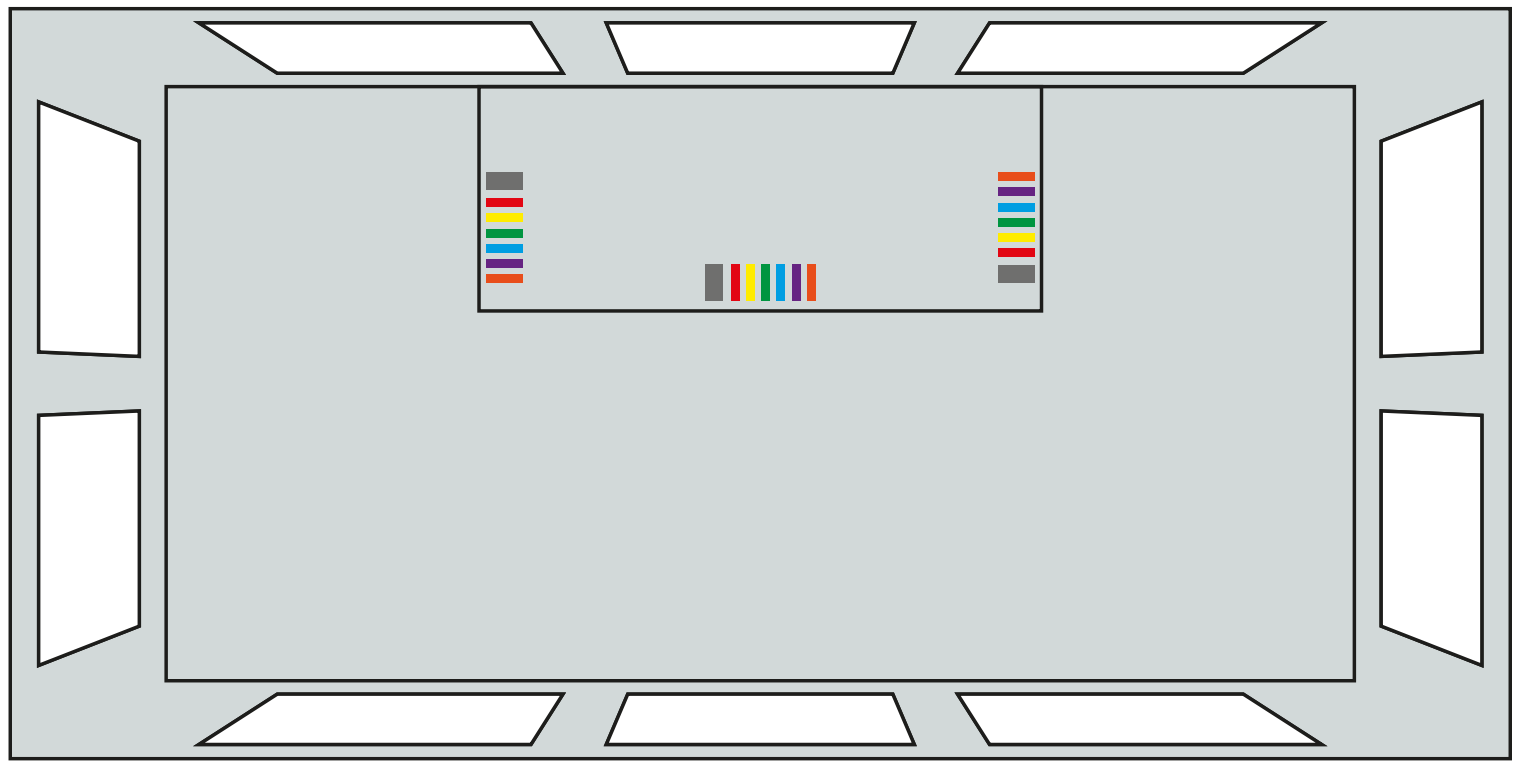

Figure 18: Bird's eye view of the VR room. Several whiteboards outline the room with a stage on one side holding the markers and erasers.

Challenges with the apparatus include making sure the VR headsets were working properly before each trial and that the live software used (VRChat) didn't have any updates that broke what the researcher was using it for. For the digital medium, the only challenge was making sure the projector was calibrated but this was easily solved early on. The physical medium did not have any challenges. 


\subsubsection{Hardware}

The hardware used for the physical medium included: post-it notes in various colours, Crayola markers and a glass whiteboard. For the digital and VR media the computers all used the same specifications. The specifications of the computers were an Intel Core 17-7700@ 4.2GHz, 32GB RAM, 64-bit Windows 10 and a GeForce GTX 1080 GPU. The digital board used the whiteboard from the physical medium with Nureva's Span system projected onto it. The VR head-mounted display was an Oculus Rift CV1.

\subsection{Procedure}

Prior to commencing the study, participants provided informed written consent via the consent form in Appendix F.

\subsubsection{Proposed Problem}

The proposed problem for the participants was to reduce kitchen waste during meal preparation. An example of the scenario map would specify the individual steps the participants take in the process of consuming a meal (Figure 18). The proposed problem was a mock challenge intended to get the participants to go through the motions and perform. However, it provided a vehicle for the participants to collaborate with one another and an opportunity for the researcher to observe their collaborative interactions within each scenario. 


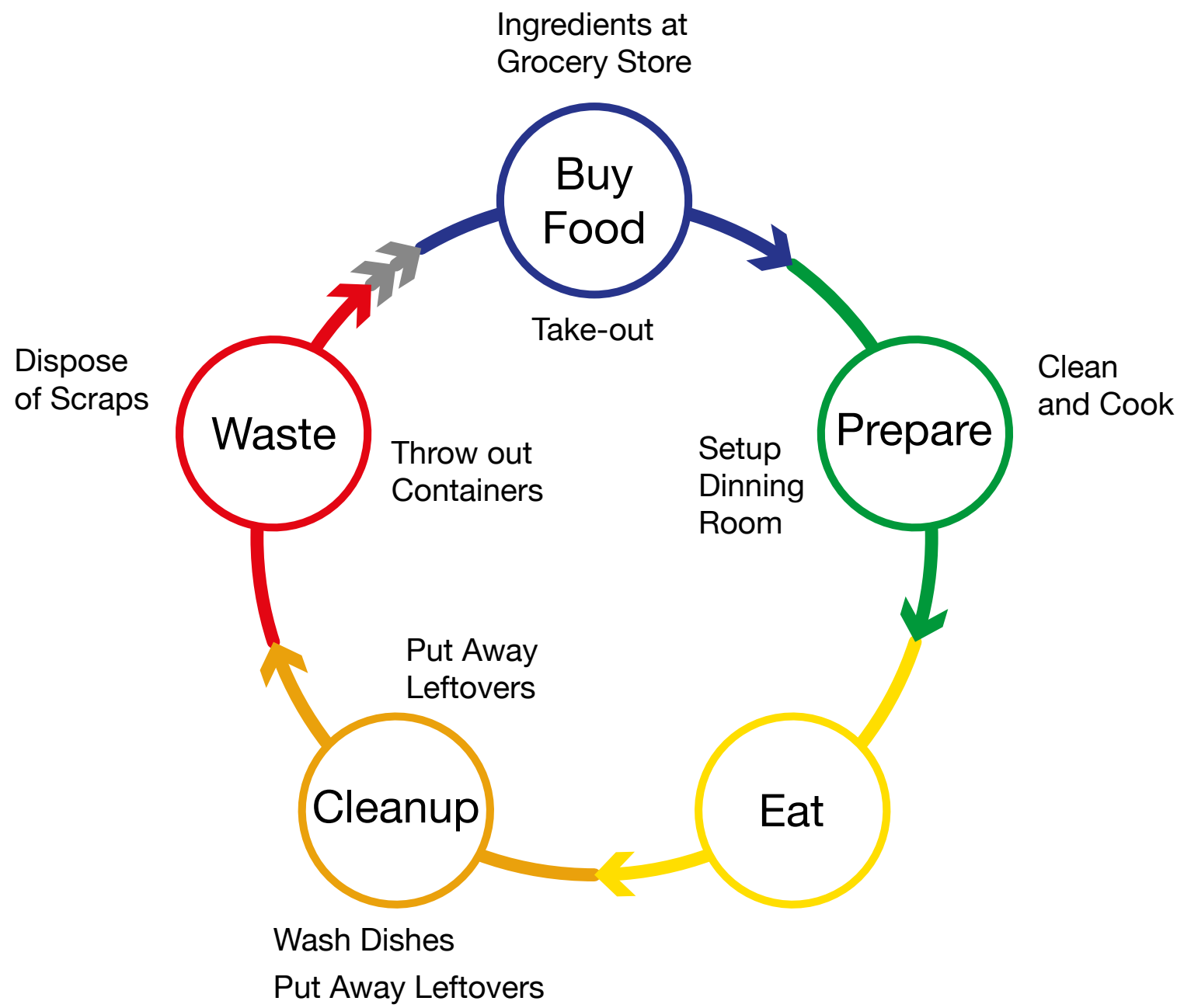

Figure 18: Food Cycle. Inner ring is an example of a food cycle using take-out food.

The outer ring is an example of some of the actions taken when cooking at home.

Developed by the researcher to visualize what process the groups followed.

\subsubsection{Hierarchy of Terms}

The terms used in this study are represented in Table 2 . Trial refers to the number of the Group where Group 1 is the same team throughout as are Groups 2 and 3. Media refers to the apparatus described in the previous section of this chapter. Exercise refers to one of three exercises which are detailed in section 3.3.4 below. Scenarios refers to the combination of media and exercises as described in section 3.3.3 below. Note that in the table below the order of combinations differs for each group. 


\begin{tabular}{|c|c|c|c|c|c|c|c|c|c|c|c|c|c|c|c|}
\hline Trial & \multicolumn{15}{|c|}{ Group } \\
\hline Media & \multicolumn{5}{|c|}{ Physical } & \multicolumn{5}{|c|}{ Digital } & \multicolumn{5}{|c|}{ VR } \\
\hline Exercise & \multicolumn{5}{|c|}{ As-is Map } & \multicolumn{5}{|c|}{ To-be Map } & \multicolumn{5}{|c|}{ Feedback Grid } \\
\hline Scenario & $\begin{array}{l}\text { Physic } \\
\text { As-is }\end{array}$ & $\begin{array}{ll}a+ \\
\text { lap }\end{array}$ & $\begin{array}{l}\text { syical } \\
\text { be } \mathrm{Mz}_{\mathrm{z}}\end{array}$ & 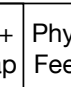 & & $\begin{array}{c}\text { Digit } \\
\text { As-is }\end{array}$ & & $\begin{array}{l}\text { igital } \\
\text { be }\end{array}$ & & $\begin{array}{l}\text { ital t+ } \\
\text { doac }\end{array}$ & & & $\begin{array}{l}\text { VR + } \\
\text { o-be Ma }\end{array}$ & & $\begin{array}{l}\text { VR }+ \\
\text { eadbac }\end{array}$ \\
\hline Steps & 1 & 2 & 3 & 4 & 5 & 1 & 2 & 3 & 4 & 5 & 1 & 2 & 3 & 4 & 5 \\
\hline
\end{tabular}

Table 2: Hierarchy of terms.

\subsubsection{Scenarios}

In this study, the word "scenario" (Table 2) refers to the media that was used in combination with an exercise, while exercise refers to the set of steps the participants followed from the IBM Design Thinking Field Guide. The IBM field guide was chosen as the reference for the scenario since IBM is a well-established company with a strong set of design thinking practices that are openly available online (IBM, 2018). This made it easy to adopt a well-known process for design thinking and follow a logical order for the participants to complete the study.

\subsubsection{Exercises}

The scenarios each consisted of one out of three exercises from the IBM Design Thinking Field Guide (IBM, 2018). All three exercises revolve around the same problem that the design thinking activity would address and the problems were always in the same order as each succeeding information generation exercise builds upon the previous one. The exercises that follow one another are: the "as-is scenario map", "to-be scenario map" and the "feedback grid" (IBM, 2018). These were chosen as they can succeed each other and build on the previous each time. The reasoning behind this order was that participants started out with the "as-is scenario map" to map out what they currently do, so not much in-depth thinking was required, and they can start right away. The second exercise of the "to-be scenario map" requires a bit of creative thinking but draws from the problems that the participants identified in the as-is exercise. Solving any of the problems found in the 
as-is contributes to this idealized "to-be scenario map". The final exercise, the "feedback grid", comes last as it serves as a review for participants. This gave them the chance to playback the solutions they came up with in the "to-be scenario map" to review what they think could work or question what the outcome would be if the changes were implemented.

The script for each exercise is attached to this document in Appendix B.

\subsection{5 “As-is Scenario Map” Steps and Details}

Participants were presented with a grid with four titles: phases, doing, thinking and feeling (Figure 20). They were instructed to fill them in with sketches or words, one thought per note, about what they currently do, think and feel while preparing a meal for themselves. They were directed to highlight questions and problems to solve later. The goal was to map their current user experience at each step of meal preparation (Appendix H).

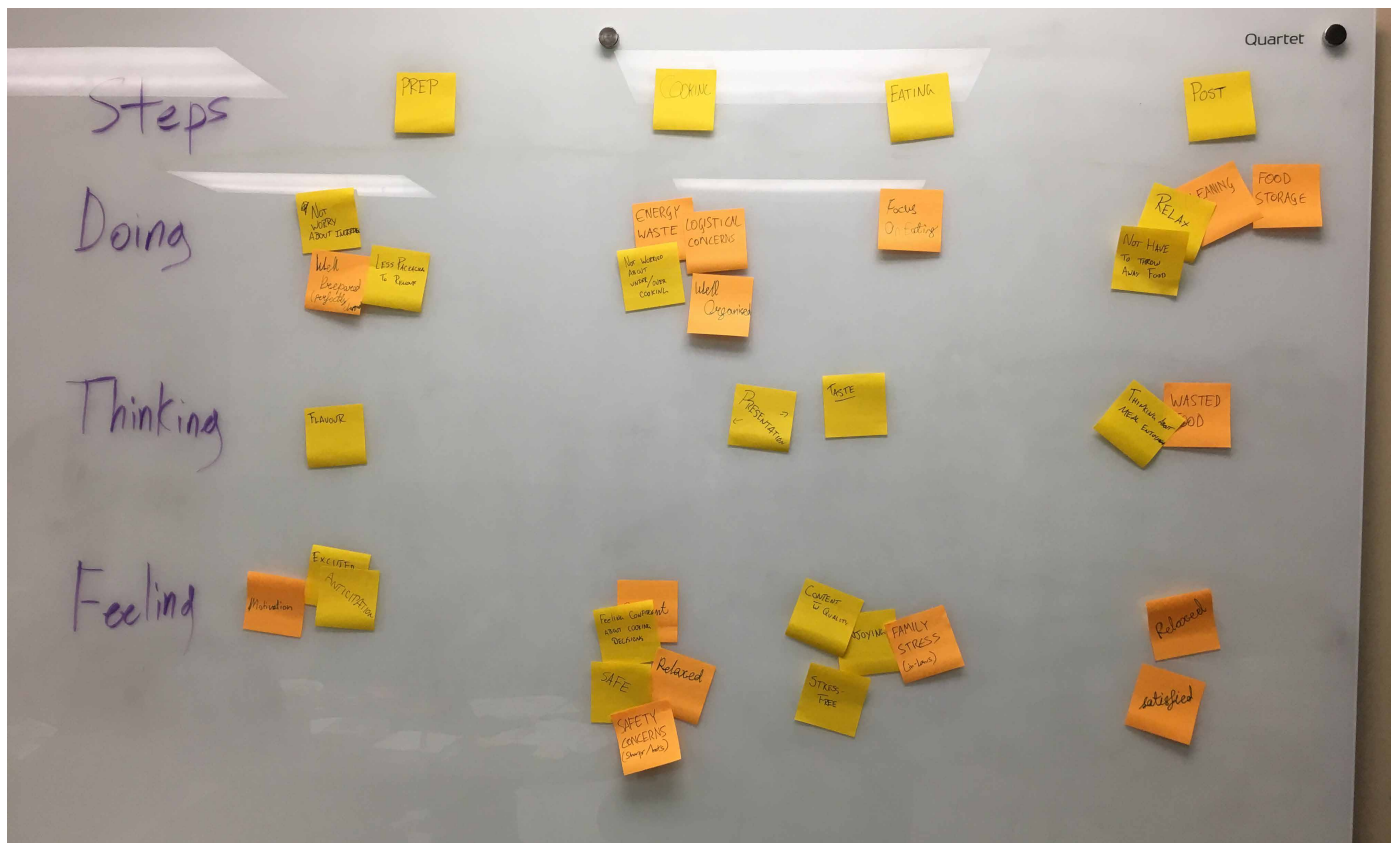

Figure 20: A finished as-is scenario map from Group 3.

\subsection{6 “To-be Scenario Map” Steps and Details}

The participants were presented with a grid using the titles: phases, doing, thinking and feeling. They were instructed to fill them in with sketches or words, one thought per note, about what they would ideally do, think and feel while preparing a meal 
for themselves. They were directed to highlight questions and problems to solve later. The goal was to have a map of what their ideal user experience is like at any step during meal preparation (see Appendix H).

Get r vommendation

forptiture rewpes besed on beftovers

USE LYS Fontroun

acess on brots Feed temelass

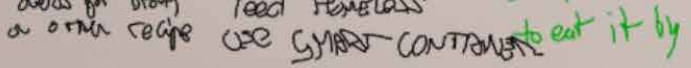
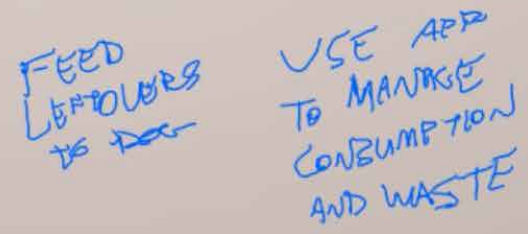

Figure 21: A part of the to-be scenario map from Group 2.

\subsubsection{Feedback Grid}

The participants were presented with a quadrant map with the following labels:

things that worked, things to change, questions they still have, and new ideas to try.

They were instructed to fill each quadrant with notes (one thought per post-it note). After everyone finished posting notes, the group clustered similar ideas together and identified patterns (see Appendix H).

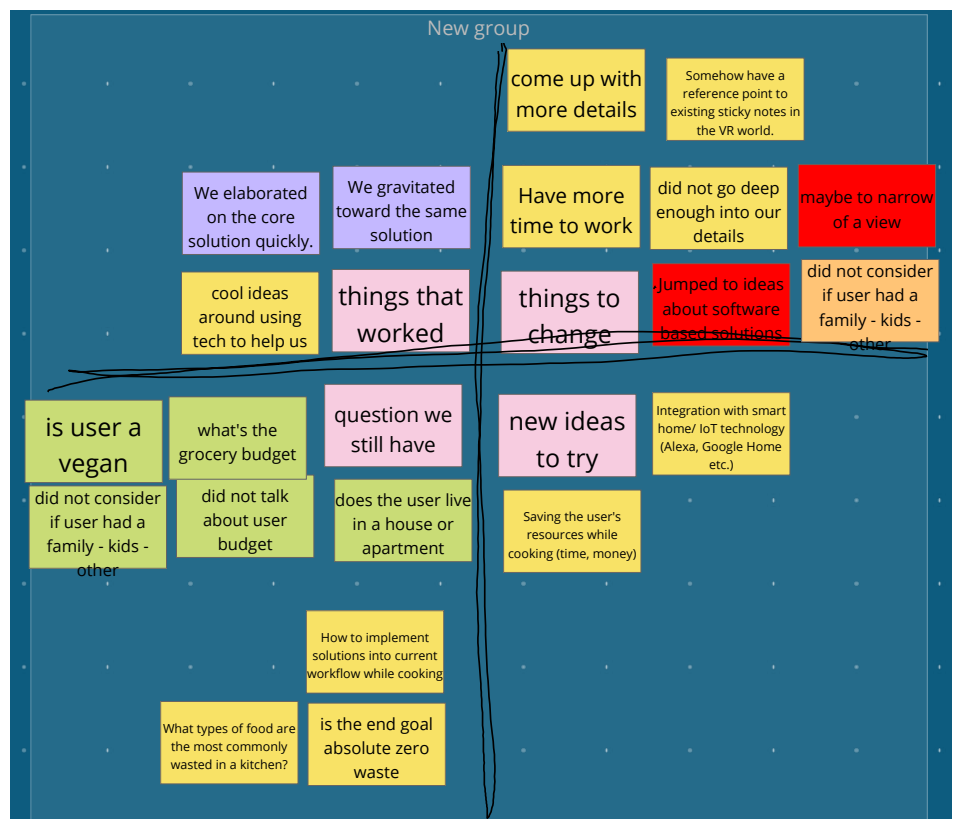

Figure 22: The feedback grid from Group 2. 


\subsubsection{Structure of Study}

The study was separated into three different scenarios (Figure 23), with each exercise taking place in different media (design thinking tool paired with media). The first exercise involved participants using their own food preparation experience in the kitchen and mapping it out in an "as-is scenario map". The second involved creating a "to-be scenario map" where the participants examined different aspects of the "as-is scenario map" to find places to improve and reduce waste. The final exercise comprised reviewing both maps and asking if what the team proposed and implemented would be successful or not if applied.

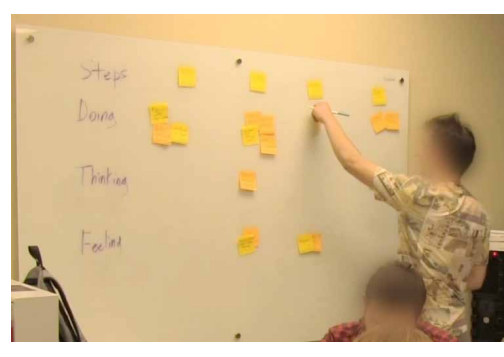

Physical

Post-it Notes

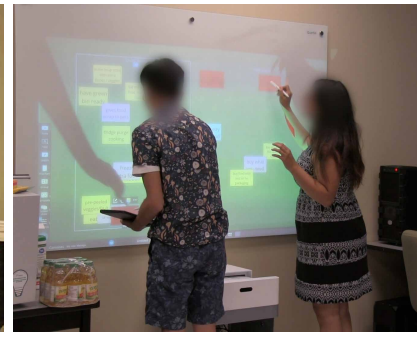

Digital

Nureva Span

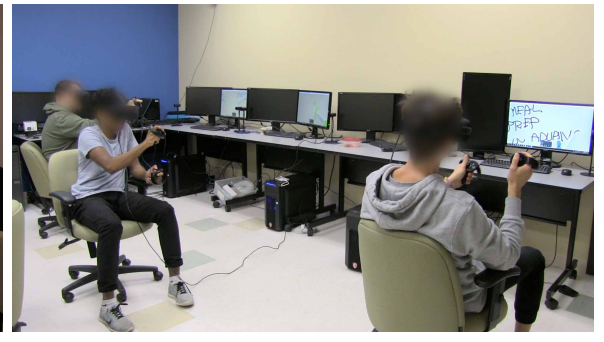

Virtual Reality

VRChat

Figure 23: The three media the participants participated in.

\section{Exercise steps:}

In all scenarios, participants were instructed to generate concepts as follows (Figure 24):

1. Generate ideas, one thought per note.

2. Post ideas to the board.

3. Cluster ideas with similar thoughts.

4. Discuss the clustered concepts.

5. Prioritize the clusters. 


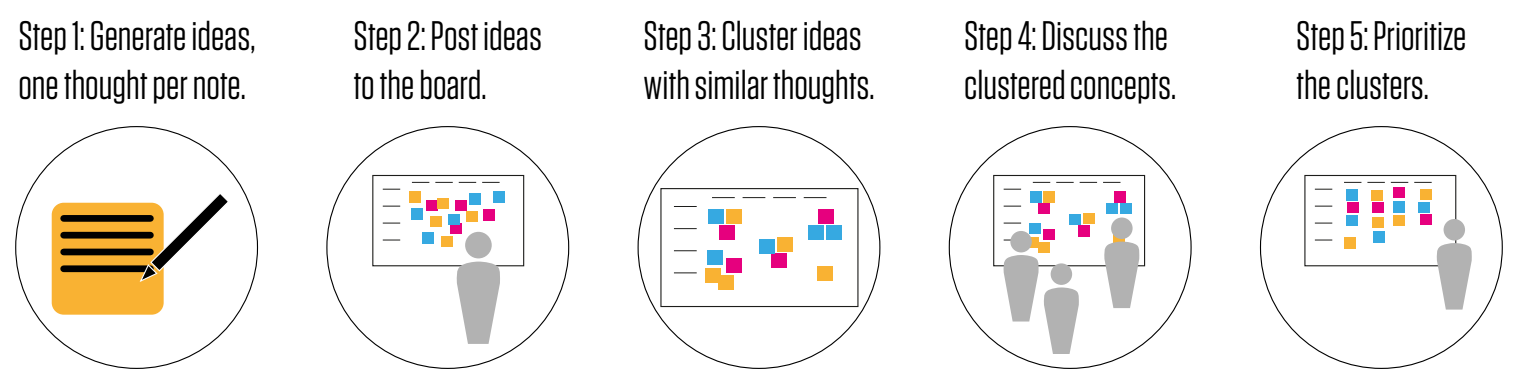

Figure 24: Exercise Steps. Derived from the IBM Design Thinking Field Guide and modified/illustrated by the researcher.

Once the clusters were prioritized and agreed upon by the members the group could move forward to the next scenario. The participants were given a few minutes before moving on to review the activity and what they just did, as the next activities would be based on the previous.

Participants were given a warm up period before starting the VR medium. The warm up period served two purposes, 1) it gave time for the participants to get accustomed to the VR environment and controls, 2) it gave the researcher time to make sure all participants were wearing the equipment properly and the study was ready to begin the VR portion. For the warm-up, participants were instructed on how to move and navigate the environment and told to try it out. Then they were told how to pick up items and draw using the markers. Once everyone was comfortable, participants were instructed to find the reset button on the stage area and press it to clean the room back to default and the activity would begin.

A quick tutorial was given for Nureva's Span software with the participants confirming they understood how to use it. The post-it notes in the physical medium did not have any instruction or tutorial on their use.

\subsubsection{Preliminary Tests}

Before the study was conducted, a pre-test of the procedure was performed by the researcher to validate the process and resolve any problematic issues before the actual study. This helped inform changes to the protocol and parts of the study. 
During the physical medium, no issues were identified and the protocol was accepted as planned.

In the digital medium, one issue occurred with the use of the touch projector. Since the apparatus for the study was setup and taken down each day, the calibration on the projector was misaligned during the next session. Because the projector needed to be moved out of the way for the physical medium, the solution to this issue was to run the calibration during the participant break beforehand to ensure accuracy during the trial.

For the VR medium, an issue was making sure the individual computers could be synchronized to the same world instance. Since the program was an online game, the instance could not be made public, even though it would make it easier to connect each computer, as it would also open up the room to random people on the internet and possibly compromise the closed, controlled nature of the study. To overcome this, the study was revised by adding a private link, shared through a text file, to the enter the room to ensure that all client computers used the same link and entered the same world. Another issue that occurred was the use of markers for writing in this VE. This program supported two different styles of writing, both in-air and on-wall, of which only the onwall was needed but neither could be disabled. To mitigate this issue with the participants mistakenly using the in-air writing style, a quick tutorial was provided during the warmup period to show both styles and why the on-wall should be used over the in-air.

\subsubsection{Data Collection}

The bulk of the data collection was by videotaping the study as the participants performed the exercises. Surveys were taken before (Appendix C) and after (Appendix D) the study was completed. The first survey consisted of demographic information (statistical), while the second was for each participant's subjective view about the activity. The reason for collecting this information was to use different data collection tools to triangulate the data by providing different perspectives that build upon each other during analysis. The second survey also gave the participants a chance to talk about any insights they had using 
the different, or in some cases new, media for completing a design thinking exercise.

To collect data throughout the process, everything the participants wrote and said was recorded. The participants were video recorded, so the researcher could take time to analyze what was going on afterward. Photos were taken to record the end result of the physical brainstorming medium, and the digital board was saved when the participants were finished. The VR medium was recorded at the desktop level using a screen capture software. This was to ensure that nothing was missed in case some clarification from the video/audio was needed. Video recording of the lab setting was also taken while the participants performed the activity. In this study video recording also includes audio recording. Any audio recording of the participant if they withdrew was removed from the study, while the sections of video that contain them were not used. There was also a person physically in the room taking notes in case the video failed.

\subsection{Study Design}

A partial counterbalanced mixed-subjects design was used, where all groups of participants ran exercises for all three media (Goodwin, 2010). This is also called a repeated measures design because the same exercises were repeated in the same order, but using a different media order for each group.

Partial counterbalancing was used because not enough groups participated in the study. It was counterbalanced by changing the order of the media for each group, as noted above at the beginning of chapter 3. Counterbalancing is a standard experimental design technique that involves changing the presentation order of conditions to offset temporal effects such as practice, learning or fatigue. In other words, each group was presented only media in a different order (Cozby \& Bates, 2015). To fully counterbalance our design would require six groups (Table 3 ). With only three groups this was considered a partial counterbalanced design (Table 4). This allowed all the participants to perform in all three media and was useful because the groups could be analyzed by themselves and then compared to the others with the averages countering any learning effect. 


\begin{tabular}{l|l|l|l|l|l|l}
$\begin{array}{l}\text { Experiment } \\
\text { Order }\end{array}$ & Trial 1 & Trial 2 & Trial 3 & Trial 4 & Trial 5 & Trial 6 \\
\hline $\begin{array}{l}\text { As-is Journey } \\
\text { Map }\end{array}$ & Physical & Physical & Digital & Digital & VR & VR \\
\hline $\begin{array}{l}\text { To-be Journey } \\
\text { Map }\end{array}$ & Digital & VR & VR & Physical & Physical & Digital \\
\hline Feedback Grid & VR & Digital & Physical & VR & Digital & Physical
\end{tabular}

Table 3: A full counterbalanced experiment order would require six trials, each one being different than the others.

\begin{tabular}{l|l|l|l} 
Experiment Order & Group 1 & Group 2 & Group 3 \\
\hline As-is Journey Map & Physical & Digital & VR \\
\hline To-be Journey Map & VR & Physical & Physical \\
\hline Feedback Grid & Digital & VR & Digital
\end{tabular}

Table 4: The partial counterbalanced experiment order used for the study. Independent variables for the study refer to the exercises and the media that all participants completed. This includes physical, digital and VR.

\subsubsection{Data Analysis}

The analysis of the study data started with reviewing the video footage and boards from all the sessions. Transcripts were made from the video recordings and used to identify some of the interactions between the participants. Once an initial viewing was completed a set of codes was created (Saldaña, 2015) for the different types of interactions that occurred. This set of codes was given the name of codex since it was referred to as an index of codes. A second viewing of the videotapes allowed timestamps to be taken for any occurrences defined in the codex. The dependent variables used were those from the codex (Appendix I): looking at the board, looking at other participants, moving a note, distracted and verbal exchange. The number of times and the percentage 
of total exercise time these interactions occur was recorded. The researcher could then synthesize the coded data, visualize it by mapping the occurrences on graphs and identifying prominent patterns that emerged from the data by looking at and comparing the graphs together side-by-side. This was done within and between groups to identify the different effects each form of media had on the participants.

Quantitative data was collected through exercise completion time, the number and kind of interactions that occur while qualitative data was collected about the subjective feeling of the participants while interacting with the media and others. The researcher analyzed the interactions within each group (i.e. Group 1 in the physical medium had more activity than Group 1 in the digital medium) and across groups (i.e. Group 1 in the physical medium had more activity than Group 3 in the physical medium).

For the first part, transcriptions, the analysis consisted of translating the video into a script that could be searched for key words and highlight specific points in the study. This aided in identifying some interactions and their occurrence.

In the second part, the codex was created by looking at the most often used interactions. This required multiple viewings to first identify what interactions would be suitable for the codex based on if and how frequently they occurred. The physical medium was viewed first because it was theorized that it had the most interactions that were easily identifiable and the other media could be based off it. Other viewings provided the opportunity to map out when and how frequently they occurred; the timestamps would then later be used for visualization and pattern identification.

The third round of analysis used the timestamps from the previous reviews to graph out what each group and scenario looked like when only codex interactions were present. Once the individual graphs were made and scaled to match each other, patterns could be identified by using full lines, blank sections and dotted lines.

The findings from each phase of the analysis build together to create the final set of data that contributes to the answer of the questions presented in the study. 


\section{Chapter 4. Findings \& Results}

This chapter presents the findings and results of the study. Analysis of the collected data provided valuable insights about interaction and collaboration. Insights answer both the proposed thesis question as well as the three sub questions. Results are organized according to each participant group, starting with the physical, then digital and lastly the VR medium.

As discussed previously, a codex (Appendix I) was written to help codify video footage and produce quantitative data. The coded behaviours include:

- looking at the board (look at board),

- looking at other participants (look at others),

- moving a note (move note),

- being distracted (distracted),

- verbal exchange (verbal exchange).

The codes were derived from both the video recordings and observations of the participants as patterns were identified and cover all of the interactions between the participants with each other and the media itself. The coded data was then plotted onto a timeline to visualize periods of activity and inactivity. This timeline illustrates a visual representation of all the scenarios in the study and provides an easy glimpse at the differences that occur between the scenarios.

\subsection{Group 1 Findings}

The first group completed the scenarios in this order: digital, physical and VR. All participants in this group already knew each other. For Group 1 all three scenarios exhibit almost the same activity times (see Figure 25, right side). The activity time is a percentage of the total time during each scenario when the participant was performing the action, seen on the left of the graph respectively. This allows for an easier comparison of the groups, especially since the duration for each scenario and group vary. In the VR medium, the time spent distracted increased dramatically. Participants had more verbal exchanges during the physical medium, as shown by the near-continuous activity in Figure 25. 

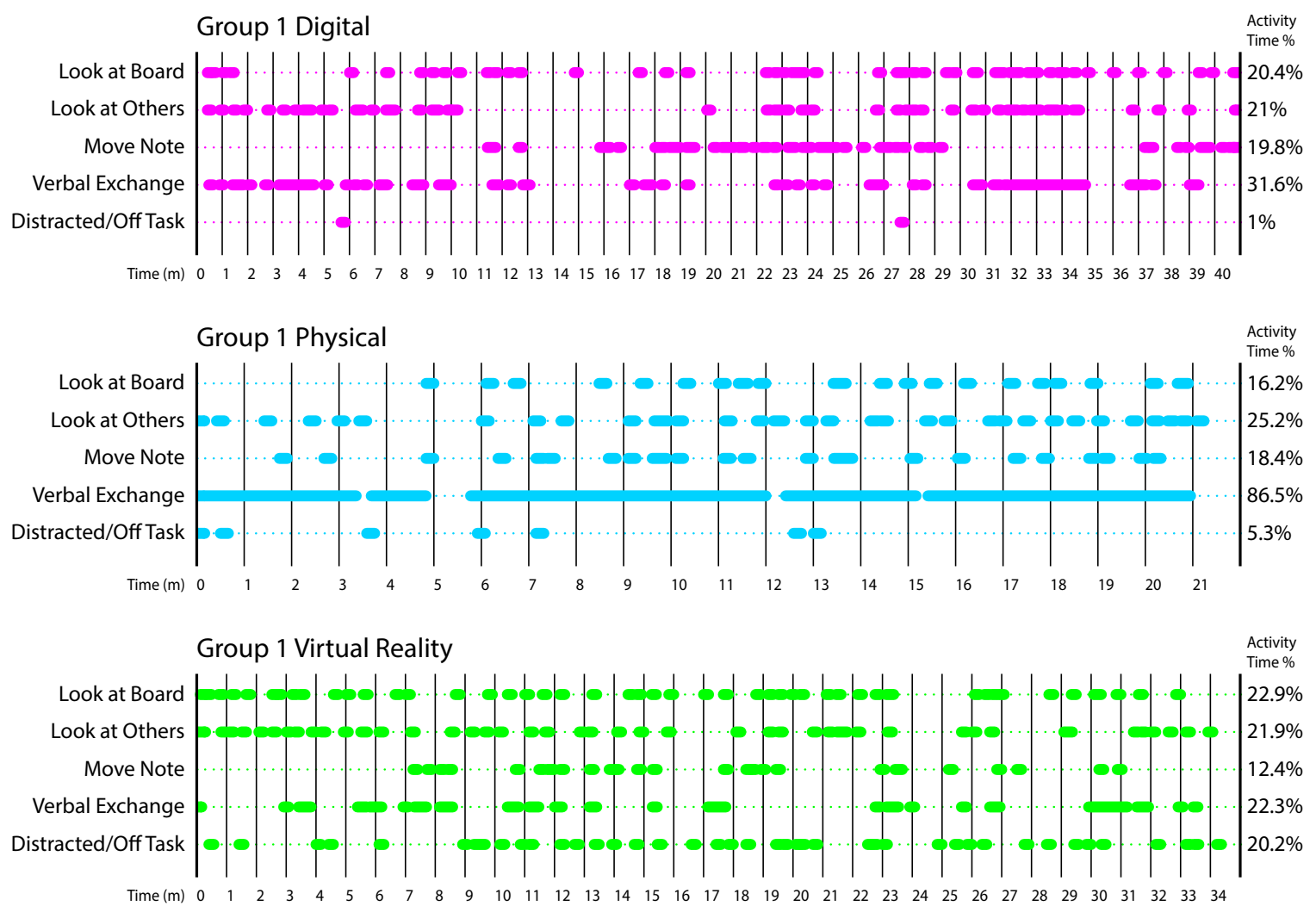

Figure 25: Activity graphs for Group 1 by time and scenario. Long lines indicate lots

of interaction without pause, while the short breaks indicate bursts of interaction.

Figure 26 depicts the same data rearranged to better compare activities across scenarios. In Figure 26, the physical medium, participants initially did not look at the board very much compared to the other scenarios. Clustering at the digital board showed a reduction in the amount that participants look at each other, but it becomes consistent for the rest of the study afterwards. For the "move note" activity, there was almost no activity during the idea generation for all three scenarios, and very little for the digital. Throughout the study the participants were actively engaging in verbal exchange. When comparing all the scenarios, distraction appears as taking up much more activity time. This suggests that VR had the participants off task more often than the 2 other scenarios. 
Group 1

Look at Board

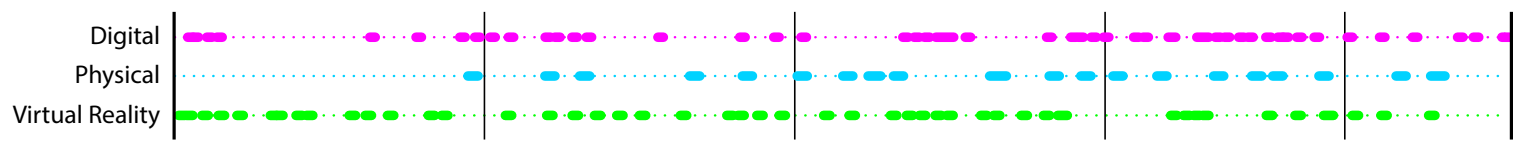

Look at Others

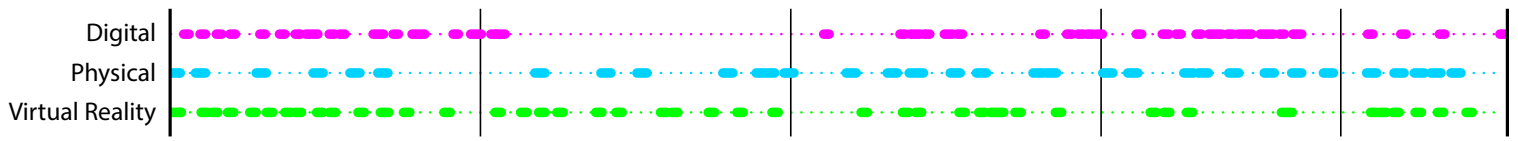

Move Note

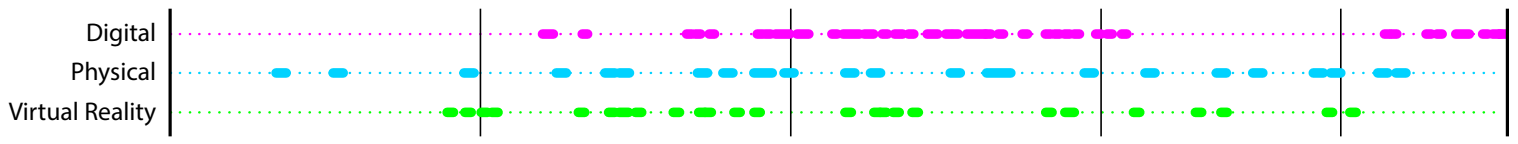

Verbal Exchange

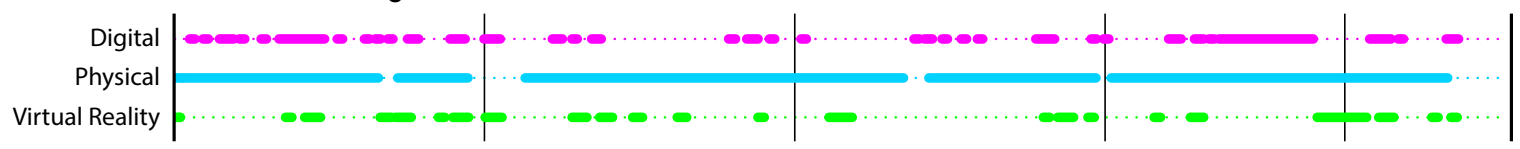

Distracted/Off Task

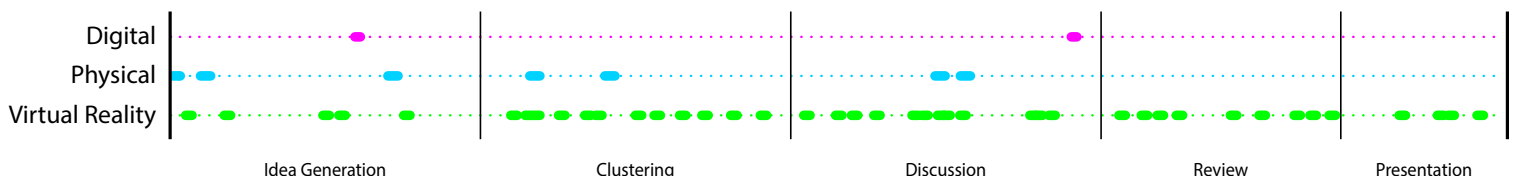

${ }^{*}$ Timelines are different so this is a general breakdown of the activty occurence during the step

Figure 26: Activity graphs for Group 1 by code and activity step. Long lines indicate lots of interaction without pause, while the short breaks indicatebursts of interaction.

\subsection{Group 2 Findings}

The second group performed the scenarios in the following order: physical, VR and then digital. All of the participants knew each other. As seen in Figure 27, this group was not distracted in any of the scenarios. They stayed on task the entire time and were very efficient with how they worked, switching from generating ideas to writing them down and moving very quickly. Most of their discussion happened toward the end of each scenario while individual thinking was performed in the first half. 

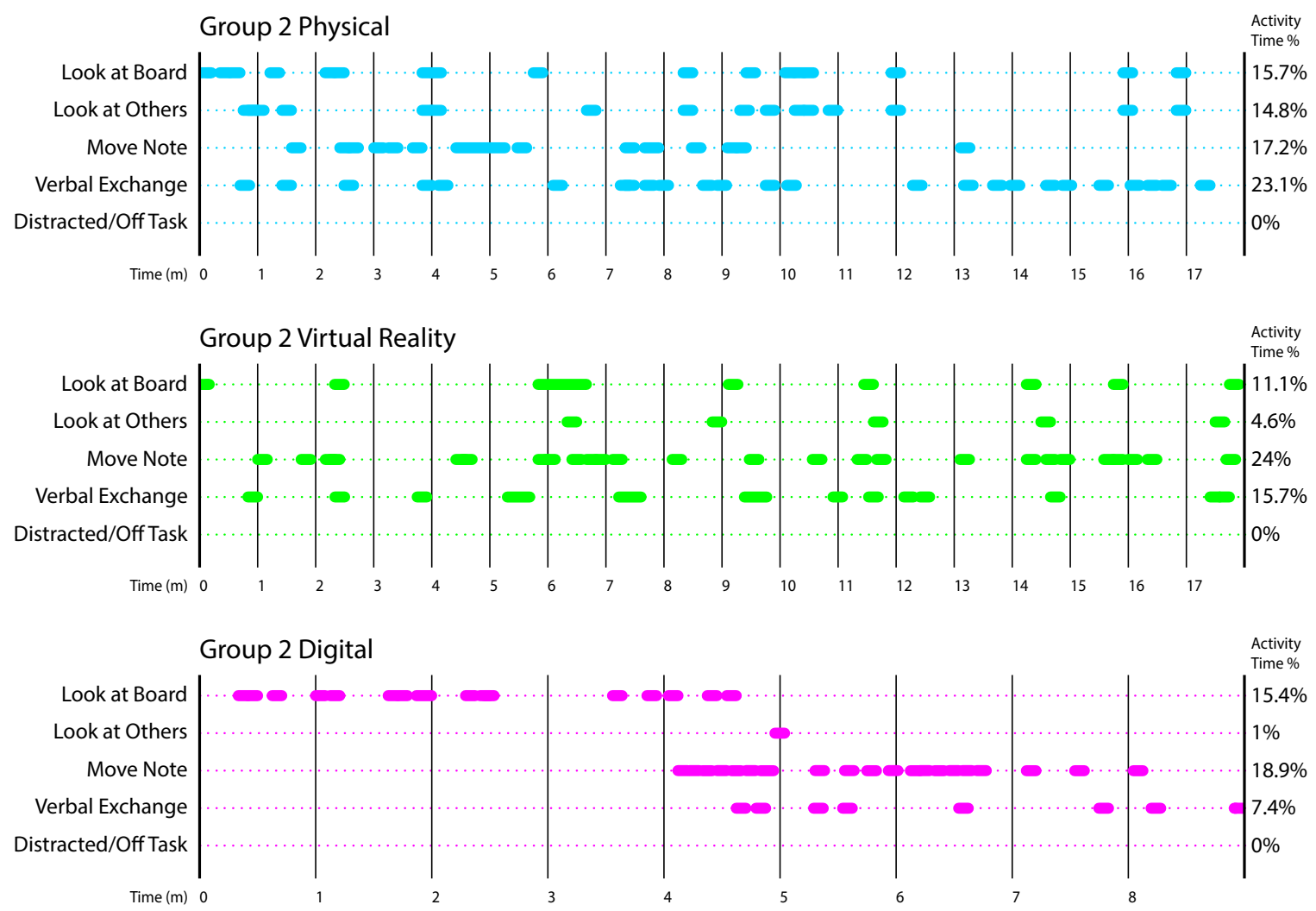

Figure 27: Activity graphs for Group 2 by time and scenario. Long breaks indicate more individual work, while the short breaks indicate bursts of interaction between the participants.

For Group 2 "look at board" was consistent for the first two scenarios, but during the digital medium they taper off. This group was very familiar with each other so other than working at the board during the physical medium they did not find the need to look at each other while communicating. Behaviour for move note was different for each scenario; with physical having more movement in the first steps, VR with consistent movement throughout and digital with movement toward the end. Verbal exchange for this group's first two scenarios was consistent as their group communicated primarily through verbal exchanges rather than looking at each other and gesturing. Digital did not have any exchange during the first two steps which may be due to everyone working at individual computers to ideate before gathering to discuss. Distracted was empty showing that no matter the media, this group did not stray from the task. 
Group 2

Look at Board

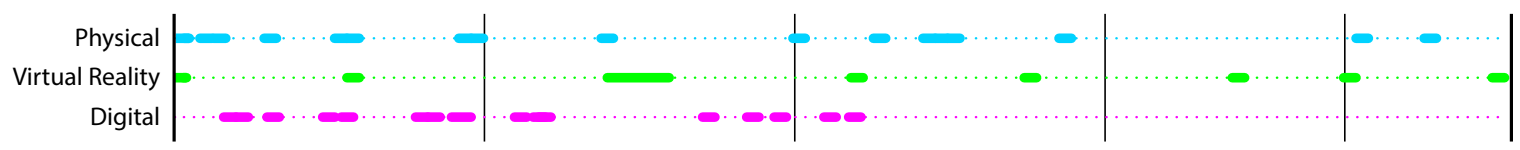

Look at Others

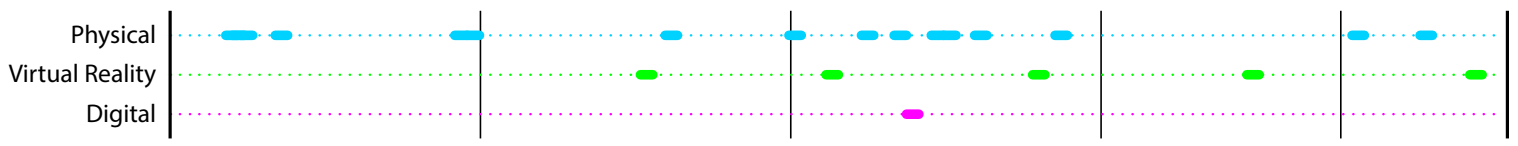

Move Note

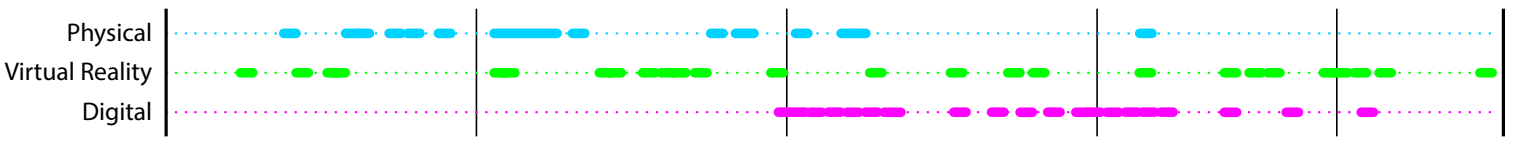

Verbal Exchange

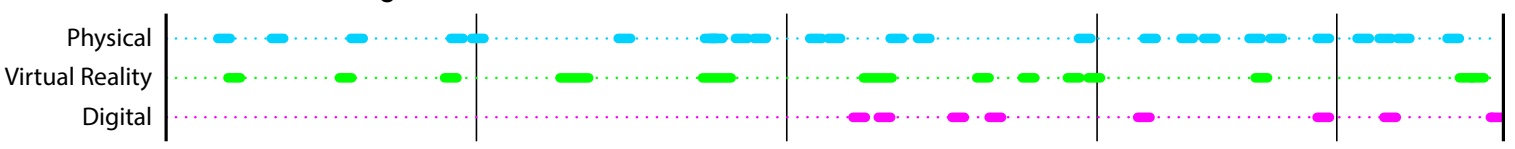

Distracted/Off Task

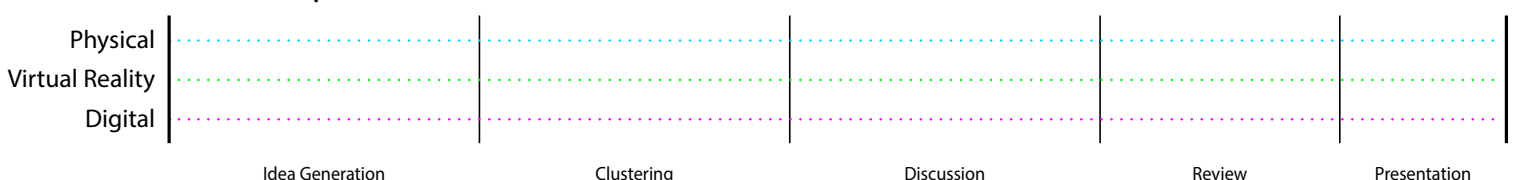

*Timelines are different so this is a general breakdown of the activty occurence during the steps

Figure 28: Activity graphs for Group 2 by code and activity step. Long lines indicate

lots of interaction without pause, while the short breaks indicate bursts of interaction.

\subsection{Group 3 Findings}

The last group to participate in the study started with VR, followed by physical and ending with the digital. This group was very talkative with each other for the last couple of scenarios, although the first was fairly quiet. Their note making and moving looks like it compensates for the reduction in verbal exchanges however as there was plenty of action during the first two scenarios. This group did not have many interactions where they looked at each other, even in the physical medium. Their distraction was much higher in virtual reality compared to the other scenarios and this may have led to the lower verbal exchange rate. 

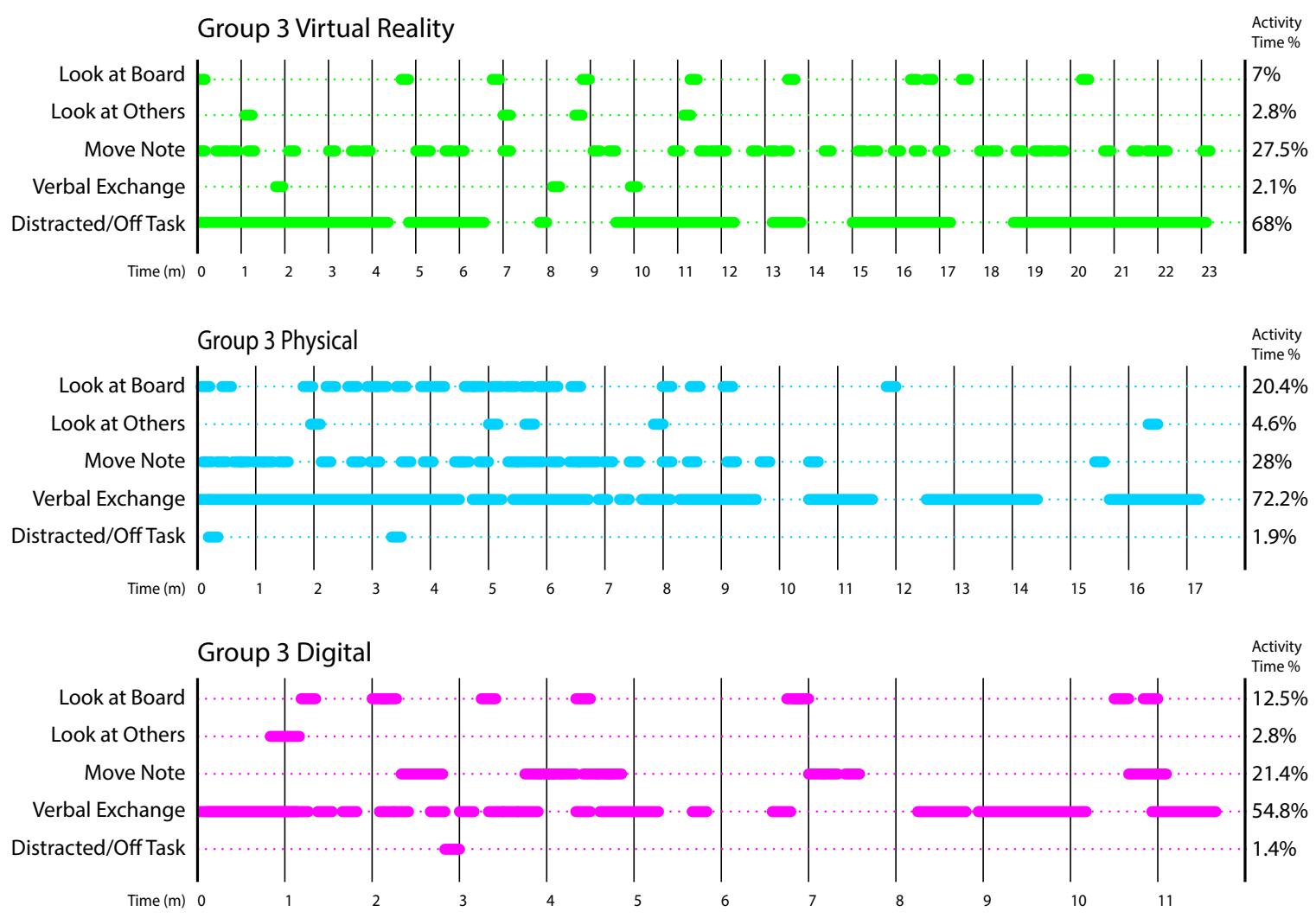

Figure 29: Activity graphs for Group 3 by time and scenario. Long lines indicate lots

of interaction without pause, while the short breaks indicate bursts of interaction.

With the graphs rearranged to show activity in the same category compared to the others, a few patterns emerge for Group 3. For look at board, this group was consistent during the review and presentation steps though there was reduced activity. Throughout the study they did not look at each other much while communicating in any of the scenarios. For move note this group was very active, especially in the VR medium, despite one of the participants needing to stop using the headset a few minutes in. Gaps during the review and presentation are visible but expected as the activity was usually close to completion and ideas were finalized at this step. Verbal exchange was almost not present in the VR medium and this was contrasted by the distraction activity that was also very high. 
Group 3

Look at Board

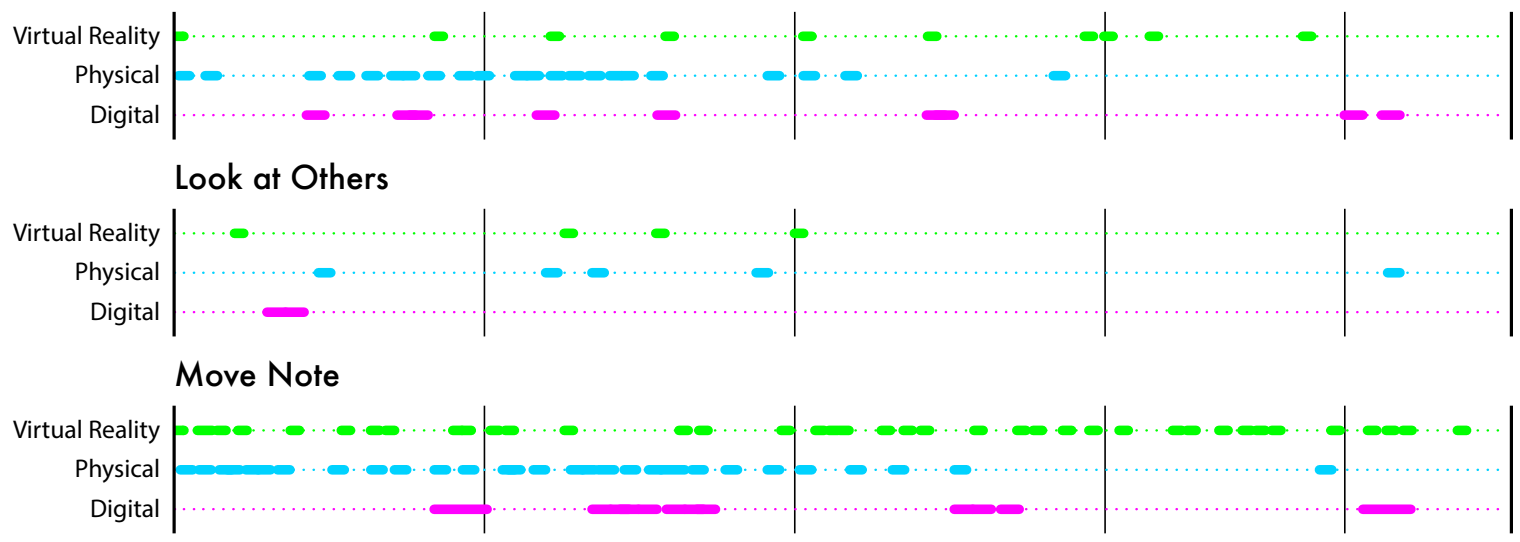

Verbal Exchange

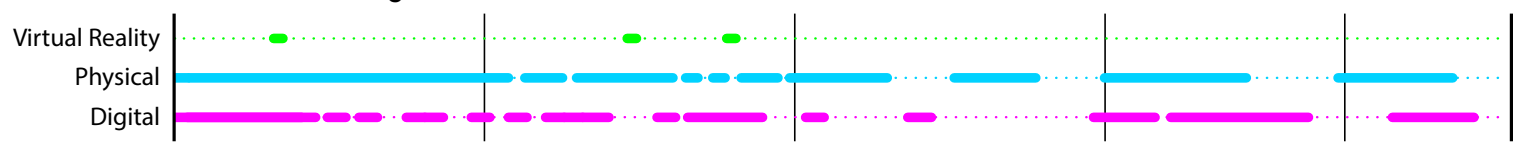

Distracted/Off Task

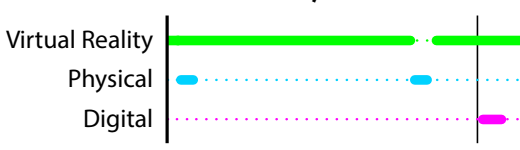

Idea Generation

Clustering

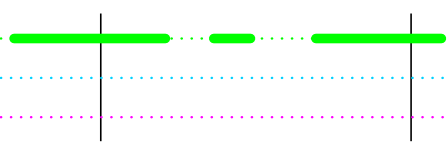

Discussion

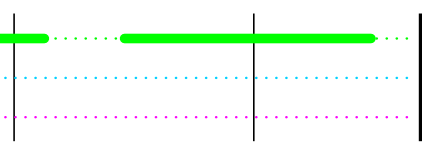

Review

*Timelines are different so this is a general breakdown of the activty occurence during the steps

Figure 30: Activity graphs for Group 2 by code and activity step. Long lines indicate lots of interaction without pause, while the short breaks indicate bursts of interaction.

\subsection{Gestures in use}

\subsubsection{Gestures Participants used, and Unique Interactions}

Gestures performed in the study consist of any physical action the participants used to communicate with each other. This could be as simple as pointing while calling something out or a much more complex gesture such as using both hands in the environment to describe something. Within the context of this study, most of the gestures revolve around creating and manipulating ideas in all three forms of media. The gestures that were used by the participants include those that were common across the groups and media, and unique ones that were specific to the group or media. In the digital and VR media these unique gestures may have been derived from the physical medium with which all of the participants had much more experience. 
As illustrated below, all of the groups used the same common gesture in each scenario, utilising the medium it was intended for while also expressing a unique gesture.

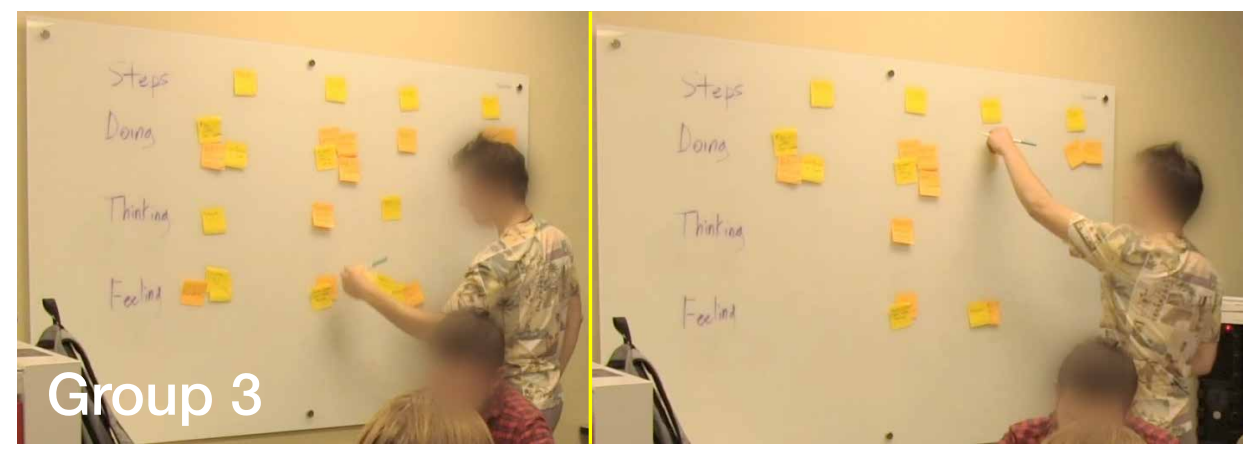

Figure 31: Common physical gesture: the most common gesture for the physical medium was placing and moving post-it notes.

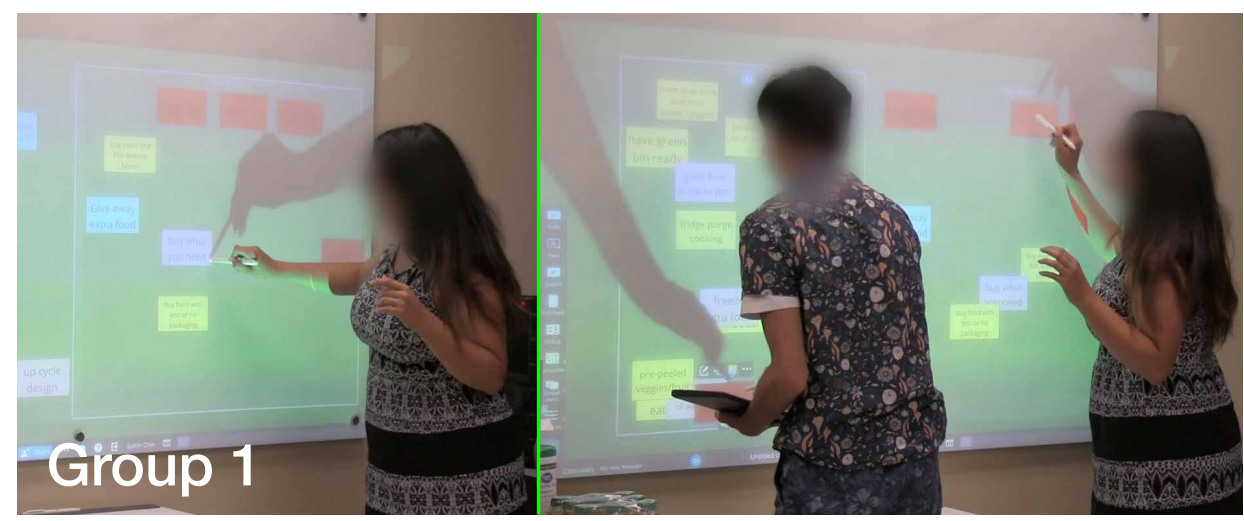

Figure 32: Most used digital gesture: during the digital medium, the most used gestures were double tapping to edit and dragging to move notes around.

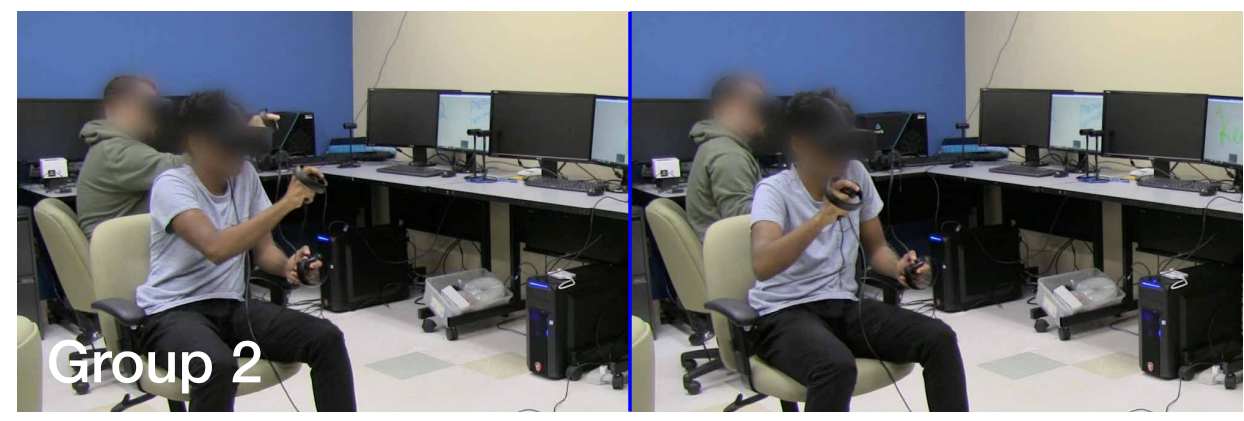

Figure 33: Most used VR gesture: in the VR medium the gesture most used was writing in the air. A few of the participants used both hands for writing, with their non-dominant hand using the eraser. 


\begin{tabular}{|c|c|c|c|c|}
\hline Interactions & $\begin{array}{l}\text { Related Gestures } \\
\text { (see below for } \\
\text { descriptions) }\end{array}$ & Group 1 & Group 2 & Group 3 \\
\hline \multicolumn{5}{|l|}{ Physical } \\
\hline Hand visualization & 1 & $\bullet$ & - & \\
\hline Placing & 2 & 0 & 0 & 0 \\
\hline Removing & 3 & • & - & - \\
\hline Board writing & 4 & & - & \\
\hline Delegation & 5 & ○ & & - \\
\hline \multicolumn{5}{|l|}{ Digital } \\
\hline Hand visualization & 1 & - & & - \\
\hline Dragging elements & 2 & • & - & - \\
\hline Tapping elements & 6 & • & $\bullet$ & 0 \\
\hline Scribbling & 4 & - & & \\
\hline Flicking elements & 6 & • & & \\
\hline Deleting & 3 & $\bullet$ & 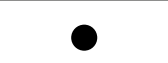 & 0 \\
\hline Delegation & 5 & - & - & \\
\hline \multicolumn{5}{|l|}{ Virtual Reality } \\
\hline Drawing & 4 & - & - & - \\
\hline Erasing & 3 & - & 0 & - \\
\hline Pointing & 7 & 0 & & \\
\hline Grabbing & 2 & 0 & - & - \\
\hline Waving & 1 & 0 & & \\
\hline Delegation & 5 & $\bullet$ & $\bullet$ & $\bullet$ \\
\hline
\end{tabular}

Table 5: Group gestures across media. Dots indicate in what group the interaction occurred. 


\subsubsection{Description of Gestures}

\section{Hand Visualization}

Using the physical medium as the control, hand visualization was a common gesture used by all participants. Similar gestures to hand visualization across the other media are also marked with a ' 1 ' in the related gestures column (Table 5). In the digital medium the participants' hands were still used to communicate. In the VR medium the participants had the use of their hands as a communication tool in the virtual world. This was most often used to catch the other user's attention by waving.

Placing

Placing refers to the action of placing notes in the Physical medium on the board and moving them around. In the 2D and 3D media the equivalent interaction would be the dragging of digital elements on the board in 2D and grabbing objects to move in 3D.

Removing

Removing is the action of taking a note off the board to discard. A similar action in 2D and 3D media would be deleting elements off the board in the digital, and using the eraser in VR, to remove any writing or drawing while in the VR world.

\section{Board Writing}

Board writing is a separate activity than placing notes, as it is harder for this to be moved around. Once written, if a note needs to be moved the writing will have to be annotated or erased and rewritten. In the other media this is referred to as scribbling with the markup tools in 2D and drawing with the virtual markers in VR.

\section{Delegation}

Delegation is the act of separating a step into parts and assigning roles. Delegation was treated the same in all media, but not all groups used delegation in each scenario.

\section{Tapping \& Flicking Elements}

These interactions do not replicate any function from the physical medium but introduce a new way to activate digital board feature capability. These features were also 
used for multi-user interactions like passing chosen notes from one side of the board to a member at the other.

\section{Pointing}

To help direct others to what the user was looking at, one group had members stick their arms out to point in the virtual environment to help keep everyone on track with the current issue being talked about.

\subsubsection{Summary of the gestures}

Most of interactions from the chart above were used by all groups. The common gestures across the groups tend to be obvious gestures that were already embedded in our mental models when it comes to interacting with physical and digital objects, such as placing and removing post-it notes, dragging and tapping elements, and grabbing and drawing in VR. In addition, Delegation was a common task that each group participated in, with one of the participants becoming the group leader and assigning roles to which the other participants readily agreed. Delegation worked better with the groups where the members knew each other and their abilities already, and not as well with the group that did not know each other.

\subsection{Survey Findings}

The study participants were designers with design thinking experience. To ensure that participants were recruited from a suitable population, surveys were used before and after to help validate that they had both design experience and knowledge of design thinking methods. The surveys consisted of multiple choice, Likert scales and open-ended questions.

\subsubsection{Multiple Choice}

The multiple-choice questions were simple yes or no questions to validate whether or not the participant was involved in a design-related field and if they had ever participated in a design thinking activity or workshop. Age was also recorded through binning. 


\begin{tabular}{|l|c|c|c|}
\hline Pre-test Questions & Yes & No & Total \\
\hline $\begin{array}{l}\text { Have you ever participated in a design activity } \\
\text { before? }\end{array}$ & 8 & 1 & 9 \\
\hline If yes to the above, did you enjoy the activity? & 8 & 0 & 8 \\
\hline Do you see value in design thinking activities? & 9 & 0 & 9 \\
\hline
\end{tabular}

\begin{tabular}{|l|c|c|c|}
\hline Post-test Questions & Yes & No & Total \\
\hline Did you enjoy the activity? & 9 & 0 & 9 \\
\hline $\begin{array}{l}\text { Did this activity change the way you see } \\
\text { design thinking activities? }\end{array}$ & 7 & 2 & 9 \\
\hline
\end{tabular}

\begin{tabular}{|l|c|c|c|c|}
\hline What is your age? & $18-21$ & $22-25$ & $26-30$ & $31+$ \\
\hline & 3 & 1 & 3 & 2 \\
\hline
\end{tabular}

Table 6: Multiple choice answers.

\subsubsection{Likert Scale Findings}

A Likert scale was used for the post-test survey to gather thoughts on the activity. The scale went from 1 (not very) to 5 (very interactive).

Can you rate how interactive you think the design activity was from 1 (not very) to 5 (very interactive)?

\begin{tabular}{|c|c|c|c|c|c|}
\hline 1 & 2 & 3 & 4 & 4.5 & 5 \\
\hline $0 \%$ & $0 \%$ & $0 \%$ & $22 \%$ & $11 \%$ & $67 \%$ \\
\hline
\end{tabular}

Table 7: Likert scale answers.

\subsubsection{Open-ended Questions Findings}

Open ended questions were used in both pre-test and post-test surveys. These questions probed the participants on what design field they were interested in, what or why they did or did not enjoy about the activity and if it changed the way they see design thinking after trying out different media for collaboration. 
Career field interests. When asked what career field the participants are interested in, answers ranged from "industrial design", "UI/UX" to "design education" and "medical device design".

Why did you enjoy the activity? Overall, the main reason seven of the participants enjoyed the activity was because of the topic concept and the use of VR. "It was fun playing with VR and the digital post it notes!" For most of the participants, this was their first time using a VR headset. For one person it "was interesting using VR for a team exercise. Can see usefulness in the future for remote collaboration".

How did this change the way you see design thinking activities? For two participants, this assured them that the previous old school methods are still relevant due to the trouble they had with the digital and VR formats. They strongly expressed their "appreciation for old school ways. No longer want to work with others in VR environments." For the other seven, it opened up new possibilities for collaboration and different ways to use new and developing technologies for design thinking and creation. "We don't need to stick to pen and sticky paper post-it notes and we can use technology to do design thinking!"

\subsection{Summary of Findings}

The researcher expected all three groups to have more similarities than differences when it came to all media interactions. The results from the three groups point toward less interaction and collaboration in the VR medium during move notes and verbal exchange varying by 1-10\% based on the activity time in Figures 25, 27 and 29. Most of the participants' interactions comprised of generating and placing 10-30 thoughts on a board. This concept was fairly easy for each of the nine participants to understand in all three media formats; participants were able to get to work right away.

Activity Graphs

Look at board appears to be consistent with slight differences of about $5 \%$ between the scenarios, except in Group 3 where the physical medium was much higher 
(takes longer) by almost $8 \%$. The participants were able to quickly identify the area and location that the notes should be posted to and organized, and it shows an end result where all groups have similar looking boards.

Look at others shows significant changes with media where across all three groups the scenario with the least activity involved the digital board. This was especially evident at the beginning where all participants were at their assigned individual computer to generate and send ideas through the web to the digital board on the other side of the room.

Move notes for all groups appeared similar when comparing the groups, however Group 2's activity during the physical and digital was opposite the 2 other groups. While they moved notes as early steps in the physical medium, they moved notes as latter steps during the digital medium.

Verbal exchange was the most used method of communication. Activity dropped only during periods of individual work such as ideating and clustering. The standout group here was Group 3. There was a lot of self-discussion during the latter steps but no discussion as a group.

Participants were distracted 20-30 times more during the VR medium than the other media for Groups 1 and 3. The one group that did not get distracted consisted of professionals with a few years of work experience, while the other groups consisted of students. This distracted behaviour was also shown by the reduction in how often the participants look at each other when speaking. There was also a tendency for the members to be more distracted during the VR medium. This higher distraction appeared to be caused by the participants exploring new-to-them unique media and experimenting with the new features they discovered while completing the activity. In the case of the first group, being friends led to joking around and playing more, while the second group of professionals treated the activity more seriously and stayed on task. 


\section{Delegation}

Delegation was a common occurrence with all groups, occurring in 7 out of 9 scenarios. Usually one person created the notes while the others generated ideas and moved ideas around to the appropriate areas. This happened during the idea generation and clustering steps and sometimes during review when deciding on how to present.

\section{Survey Data}

Data from the surveys show that designers with prior experience with design thinking activities who participated in the study enjoyed participating in the study. They found value in doing these kinds of exercises and found this experience changed the way they think about design thinking activities. All 9 participants found the exercises very interactive.

For the open-ended questions, participants related the activity to previous group collaborations they participated in that showed both old and new technologies can be used for design thinking. The comment, "Can see the future of design thinking moving to AR", was mentioned by participants. 


\section{Chapter 5. Discussion}

This chapter discusses the study's results and compares and contrasts them to the literature. Results are first discussed in the context of the secondary questions and are organized according to research questions, beginning with the sub-questions.

\subsection{First Sub Research Question}

The first research question of this study is:

What are the interactions that designers in design thinking sessions use in these environments now?

This question was developed to better understand what interactions are currently being used in design thinking experiences. For this purpose, the answer to this question was based on the findings gathered from the physical brainstorming medium as this was the medium that was used as a control for the others. The discussion starts with interactions observed of the three groups during the physical medium and how similar or different they were to each other. Following, it considers what the study was missing from the literature and what, if any, new interactions were observed.

After the design thinking study was completed and the interactions that emerged were identified, an affinity diagram was used to map out the interactions and find relationships between the media. This led to the discovery of interactions between participants and the media. The following affinity diagram (Figure 34) shows the three media with the distinct interactions observed in grey. Interactions that were common between two or more groups or had very similar interactions were grouped and recoloured. The affinity diagram shows the interactions that may be impoverished and could benefit from suggestions to improve the features in the medium. 


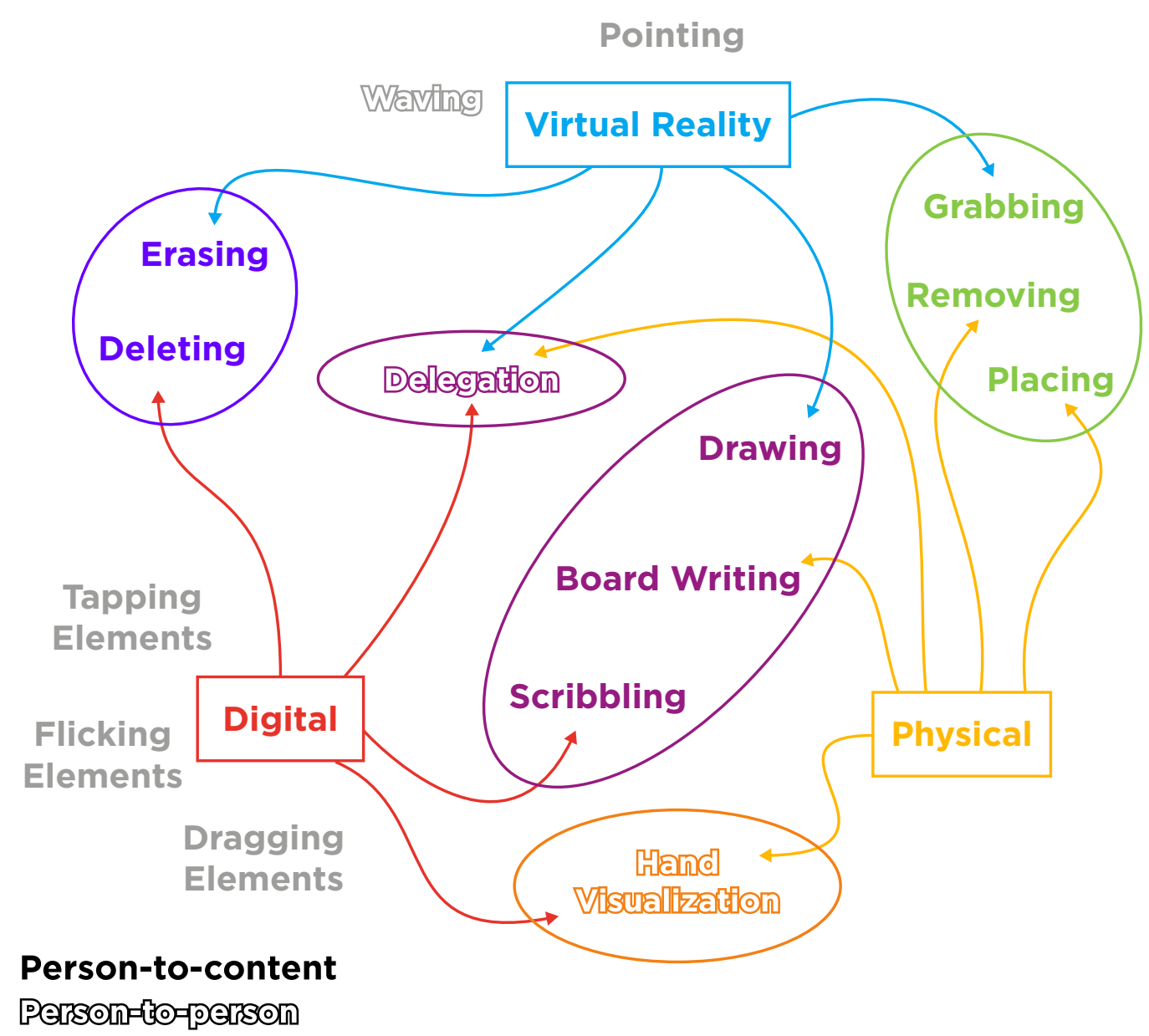

Figure 34: Interaction and gesture affinity diagram. This affinity diagram shows how the three media have related interactions and gestures.

\subsubsection{Familiar Interactions Across Media}

Finding out what designers use currently (2019) can help us understand why particular actions were taken when the group members were presented with the new scenarios involving different media. This would explain what (LaViola Jr., 2017) says when talking about designing complex 3D interaction UIs. He talks about making the UIs in a way that uses familiar components for users to understand.

The most common (eight of nine participants) interactions observed included placing their own notes and removing each other's notes an average of about three times per minute. Participants were also observed using their hands to communicate visually throughout. For example, participants used their hands to describe scale, or mimic what 
a product/concept would look like. Sometimes this gesturing was just an automatic response - to help them process rather than communicate - so it was not necessary that other participants see and gesture in return.

Eye contact when the participants look at each other or look at the board and verbal exchanges were also important for participants trying to get their ideas recorded and understood by each other. Verbal exchange took up as much as $86.5 \%$ of the total scenario time for one group. Although participants were given instructions for each exercise, the specifics of how to complete the steps using the media tools, such as post-it notes, digital notes or VR writing, were not defined. As Kolko (2011) states that, interaction design can be reliant on the participant and the context of the product, the researcher expected to see the participants interpret the tools similarly across the groups to achieve the steps. This was interesting because the participants were given the freedom to use the tools any way they wanted to accomplish the steps, but still used very similar ways.

For example, in the digital medium participants started at the client computers but were told that they could add notes directly from the board. Even after the participants started to sort and cluster the notes on the board all of the participants went back to their computer to write up more before returning to the group. This could be interpreted as it was easier to type the notes up on the computer rather than tap them out on the digital board's interactive surface keyboard. It could also indicate a lack of familiarity with the digital board, as all of the participants were comfortable typing on a physical keyboard (with touch typing) but not on a projected screen (forced pecking). It was clear that some participants experienced some trouble using the digital keyboard. This means either the media or the participants were not taking advantage of the extra dimension (the digital board can be scrolled for $20 \mathrm{ft}+$ of board space) and no benefit to having a digital keyboard present was gained. This was just like simple 2D panels that (Patel et al., 2006) suggested when he referred to developers and 3D menus. This does not rule out that the UI itself could use improvement with the input methods. Future design considerations would 
include this as part of testing to see what input method is not only preferred, but easier. This also applies to VR where participants may prefer to use a custom virtual keyboard if such one existed for VR (Figure 35) rather than writing out their thoughts and ideas.

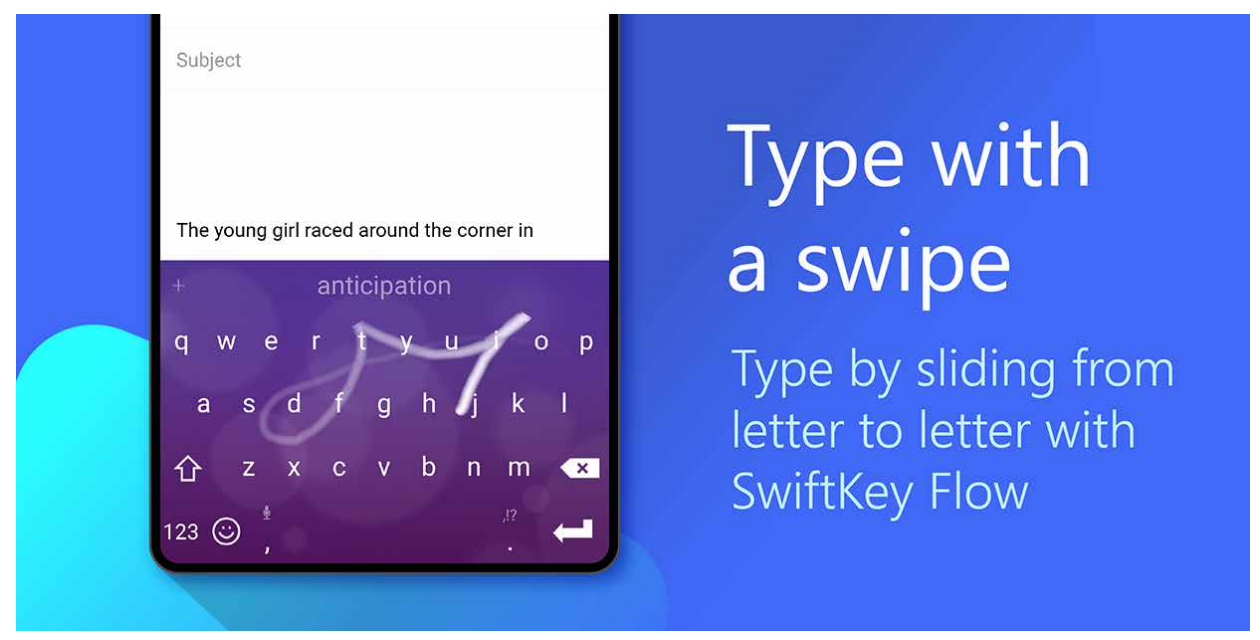

Figure 35: Mobile swipe keyboard example. An example of an alternative keyboard for mobile devices where swipes can be used to type out words and sentences that could have a VR counterpart (Swiftkey, 2019).

Another example was how the ideas were spread out in VR. Most of the participants chose a separate board for each group/step or used a separate space in the room to write an orb of text (Figure 36). This resulted in writing that looked like it wrapped around a sphere that only the author would be able to see and read properly due to the specific viewing angle needed to see the alignment of letters. Seeing the other participants react to the orb of text, and it being unreadable from almost every other perspective (Figure 36), changed how the participant who wrote it felt about their method of writing the idea down. Reactions ranged from "oh I thought I was writing on the board", to sarcastic "well, you can see it fine if you just stand here". Mason (2001) remarks that integrating sensory information into a virtual environment is required to make it feel natural, but in this situation the writing felt natural to users, even if it was not comprehensible during the scenario. Although writing in free space requires holding a button on the controller, marking the board does not. 


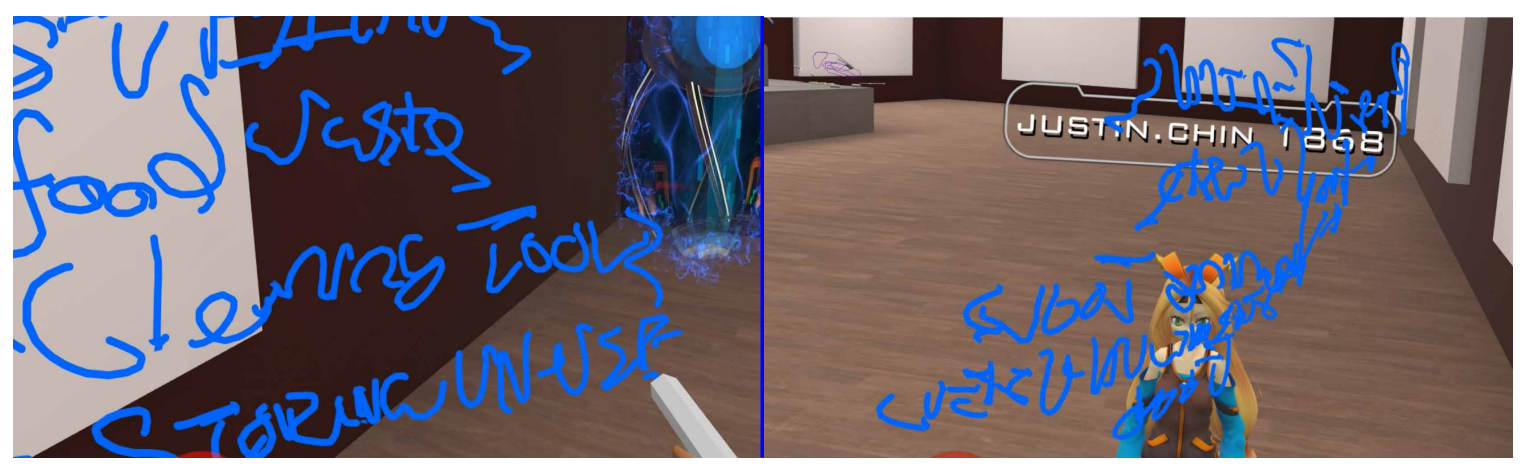

Figure 36: Orb of text from the study. (A) An orb of text written from the authors perspective in VR. The text wraps around them as they pivot while writing. (B) The orb of text from another participant's view showing the wrapping and unreadability from any other angle.

Patterns were observed that suggest similarities between the physical and 2D media. This includes participants using their hands to help visually explain some of the concepts, and the process of moving and removing notes as well as discussions after some notes are made to create new ones. For two groups while in VR, participants were observed completing one section after a short discussion and then moving on to the next. The last group typically had one participant still reading or writing from the previous step before joining the other participants. During the sections there would be writing and erasing but no movement of the text to another board. This did not have an effect on the group's work but could make the process of review more cumbersome.

In short, the most common gesture used by the participants during person-tocontent interactions involved moving notes around. For person-to-person, the interaction with the most activity was verbal exchange followed by hand-based gesturing.

\subsubsection{Missing and New Interactions}

During the VR medium interactions that were missing from this, but occurred in the other two, included hand visualization and the moving of ideas as participants never bothered to use them. The latter can be attributed to limitations of the VRChat. Ideas can take the form of anything the participant creates, whether it was a one-word statement or 
a complete sentence, it could even be a drawing. For the hand visualization, participants did have quite a range of hand manipulation, as the software supported articulation of the fingers - meaning they were able to control how most of the fingers bent, similar to what it is like in person. None of the participants appeared to use this VR feature to help explain any of the ideas being talked about even though they were specifically told what features and functions they could use during the warm-up period. To solve the moving of notes and ideas participants preferred to reference the idea by writing a shorter version of it. Rewriting the whole text would either take too long or be too much work, so it seemed to be easier to reference the text while writing the gist of it. Not moving the text might even be the better way since it will still have the context of its original place by referencing. In the literature there was no mention of the shortcuts that these users preferred to take.

\subsection{Second Sub Research Question}

The second research question of this study is:

What are the tools for reproducing face-to-face interactions that are used now in digital and/or virtual environments?

Face-to-face interactions are an important part of design thinking activities and when moving to another medium it is important that the tools to accomplish the same steps in a similar way are available (Swing, 2000) so that the process used can follow (an

already understood) physical medium. If the current design thinking collaboration methods need to change to fit the medium it could become a new method of its own. The data to answer this question comes from observation of the participants during the scenario trials.

The kind of interactions that were observed in the physical medium include hand visualization, placing and removing notes, board writing, eye contact and delegation. All of these interactions were considered for reproduction, or at the very least a similar interaction in VR. When it comes to the tools used for the reproduction we need to look at whether any tools were used for the physical version and if a similar tool was available in VR. This was where the warm-up period may be able to reduce some of the 
stress (Swing, 2000) as the participants learn some of the features and their similarities. Interpretation of the participant observations during the exercises, especially during the VR medium also supports the use of a warm-up period to introduce new tools.

The tools used for placing and removing notes were the physical post-it notes themselves and the markers used for writing. Currently the most similar tools available in VRChat were virtual markers, which lack the ability to write on something moveable. Allowing notes to be moved freely is important as it reduces the learning curve (Tiwari \& Holtham, 1998) because where they were initially placed may not be the best spot, or it may get clustered with other similar ideas like in the other media. Making the notes moveable also prevents clutter on the board from duplicate notes being written to recategorize during clustering.

For hand visualization, the tool used in the physical medium would be the user's arms and hands, while the tool available in VR was the touch controllers that were able to mimic the look of the user's hands based on how they press and hold the controller. It was not as articulate as a real person's hand, but it was accurate enough to point using different fingers and make different gestures. Board writing and delegation worked the same between both media as the tools needed for its operation existed in the same fashion. The virtual markers were able to write on the virtual boards in the environment and the participants were able to speak with each other to delegate some of the steps.

\subsubsection{Tools used Across Media}

Moving from physical to digital to VR should be a seamless transition even though it clearly is not (Billinghurst, 2002), as the basic tools for completing the same steps were present in all media. For writing and placing notes in the physical medium, the participants were instructed to use post-it notes. This transitioned to digital with the use of web-based notes where the participants could type the notes and move them by dragging on a physical board in the room. In the VR medium, the available tools consisted of virtual markers and erasers that allow hand-written text on virtual boards. 
A limitation of available programs discussed further (see Chapter 6) explains why the disconnect between being able to move the notes freely in the first two media to a third, more restricted medium caused some confusion for six of the participants. They were, however, able to overcome the restriction quickly to complete the steps on time by just writing snippets or references to the original. This indicates an opportunity for improvement in the program design of a VR tool that mimics the same interaction for writing post-it notes that people are comfortable using in physical and digital, and that could be potentially better than face-to-face (Miller, 2010).

\subsubsection{Interactions with the Media}

In the context of using the media, the interactions can be seen as both interfacing with the media and communication with other participants and also includes reactions to the feedback from both kinds of interactions. In the physical medium participants see and hear the activity of more and more notes going up on the whiteboard. In the digital they have less sensory info, because they only see the accumulation of notes when they turn to look at the board. In the VR medium there were no cues at all; the only way they knew someone had written something was to look around periodically to see the board. In VR they have a limited field of view and only audio of the other participants speaking.

During the study training period participants were told that the marker and erasers in the middle were for writing on the walls. Once the first participant started free drawing in 3D space the others quickly joined them in figuring out how the controls worked with the markers. After some instruction most participants could use the tool easily. Some participants had difficulty differentiating between the in-air action that occurred at each place the marker moved and writing that appeared only on the walls. After getting visual feedback by bringing the markers near the wall to write, all of the participants understood how it worked, though some participants preferred to keep free writing in the 3D space. The resulting text was not always readable from different angles and required other participants to move into a certain position to clearly read it. 
In VR it was easy to ignore the viewpoint of everyone else and without the constraint of having to move while writing on a board, a few users started spinning their view around themselves instead of moving along the board. This was different from the other media due to the physical limits, such as arm reach in the physical medium and the flat scrolling nature of the digital board. The learning curve (Swing, 2000; Tiwari \& Holtham, 1998) here was quickly overcome as the participants start to communicate while in VR and mention that some of the writing was in the wrong place or unreadable. It was usually after a situation like this happens that the participants learn the real difference between the in-air writing in VR and the board-specific writing in the environment.

\subsubsection{Interactions with Other Participants}

The interactions participants had with each other included speaking and gesturing. Since the activity was collaborative in nature, it was important that group members communicate and work with each other. This was the same for all scenarios and the groups communicated and interacted well with each other. Speaking with each other was tracked through verbal exchanges (Figures 25-30), where activity was recorded when a speaker was heard by another participant or a dialogue took place between two or more members.

Gestures between participants were used for communicating ideas or concepts when descriptions were not enough to get the idea across. They were used for more abstract ideas and occurred mostly during the physical and digital media. The VR medium was capable of gesturing to a certain extent but did not see much action by the participants. This may be due to a mismatch in a common frame of reference (Chellali et al., 2008). The avatars being used did not match the physical size of some participants. The size of the avatars themselves had a direct influence on the viewpoint for the participants. i.e. a very short child-like avatar would make the participant feel like they were kneeling in VR while a very tall monstrous avatar would make them feel like they were on stilts. This disconnect was pointed out by a participant in Group 2 when the virtual hands of one participant were in the air and out of bounds of the avatar model. 


\subsubsection{Interactions with both Media and Participants}

The interactions that involved both the media and participants happened during move note, where moving each other's notes occurred in both physical and digital media. In the VR medium the interactions involved the tools used for writing and erasing the notes, as well as the virtual markers and erasers. In the physical medium this situation would commonly occur when participants were sticking post-it notes to the board and moving them around (Brown, 2009). These interactions were very quick (i.e. took only a few seconds) and typically involve one participant saying "I think that should go here, not there" to another participant while moving a note. Similar situations were observed in the digital medium.

During the VR medium these interactions occurred when sharing the tools between participants. Because the markers were coloured, one group used the colours to represent different topics during the scenario. This meant that the participants would have to share the markers. To share the markers involved one participant dropping the marker in the VR space and letting the other pick it up. It was easy for the participants to see if someone was holding one of these tools, so they know who had what.

\subsubsection{Summary}

The tools used for all three media were very similar and went from post-it notes that could be stuck to a whiteboard to digital notes moved on a digital board to freehand writing in a virtual room. Similar tools made the transition for participants easier as the media changed, making it straightforward to compare across the media. Exercise completion based on time was consistent within the groups and once the first scenario, whether it be physical, digital or VR, was completed the other two were easier to understand. Participants in all three groups slowed down a little when it came to the VR medium, but it also did not take long for them to acclimate and stay on track. Eye contact was minimal for everyone except the one group where the members knew each other. 
Different types of interactions occurred during the study, interactions between the media and other participants. Reactions from participants to both feedback and the interactions were observed as well. Cues from the environment in all media help inform the participants of what was going on and how the group as a whole was working together. A warm-up period given in the lesser-known media let the participants play around and figure out how the program worked, and helped them figure out the controls needed to complete the activity. Behaviour in VR was different compared to the other two media: the participants moved and personal perspective within the environment was treated differently in VR. They worked in a less collaborative way.

\subsection{Third Research Question}

The third research question of this study is:

Are there preferred visual, auditory \& haptic feedback mechanisms to enhance collaboration?

Feedback mechanisms refer to anything that allows the participants to know that what they posted can be seen and referenced by the others. For example, when a participant physically sticks a post-it note to a board, the other participants can see and hear when the note is posted to the board. When other digital media are used the feedback needs to assure each participant that others will be able to view what they have done, whether through small status icons or other visual stimuli. It is through this feedback that the participants learn faster and can keep up with one another better (Glencross, Chalmers, Lin, Otaduy, \& Gutierrez, 2006). This is due to the information not travelling from person to person like physical formats but rather from client to server to another client during digital interactions.

\subsubsection{Feedback Mechanisms used in the Study}

The feedback mechanisms used were limited by the software itself as nothing was modified for the study. In the physical brainstorming medium, visual feedback resulted from participants looking at the board when notes were placed. Auditory feedback was 
received in the form of hearing the sounds of marker caps opening and closing, and the slap of a post-it on the board. The reactions to hearing feedback resulted in participants looking up to see the notes accumulating on the board on an average of five times per step for the first couple of steps.

In the digital medium feedback was given only when a participant visually confirmed that the digital note appeared on the board (Figure 37). During the digital medium participants would frequently look at the board (Figure 38) to confirm what they sent was posted as well as to see what the others had posted. The board also had a visual indicator to show how many notes in total were sent to the board. The visual indicator allowed participants to know how fast the others were contributing to the board. This occurred every time participants went up to the board to start moving notes around. The only indication of another participant posting notes was the clacking of keyboards as participants typed out quick bursts and paused in between, a limitation noted later.

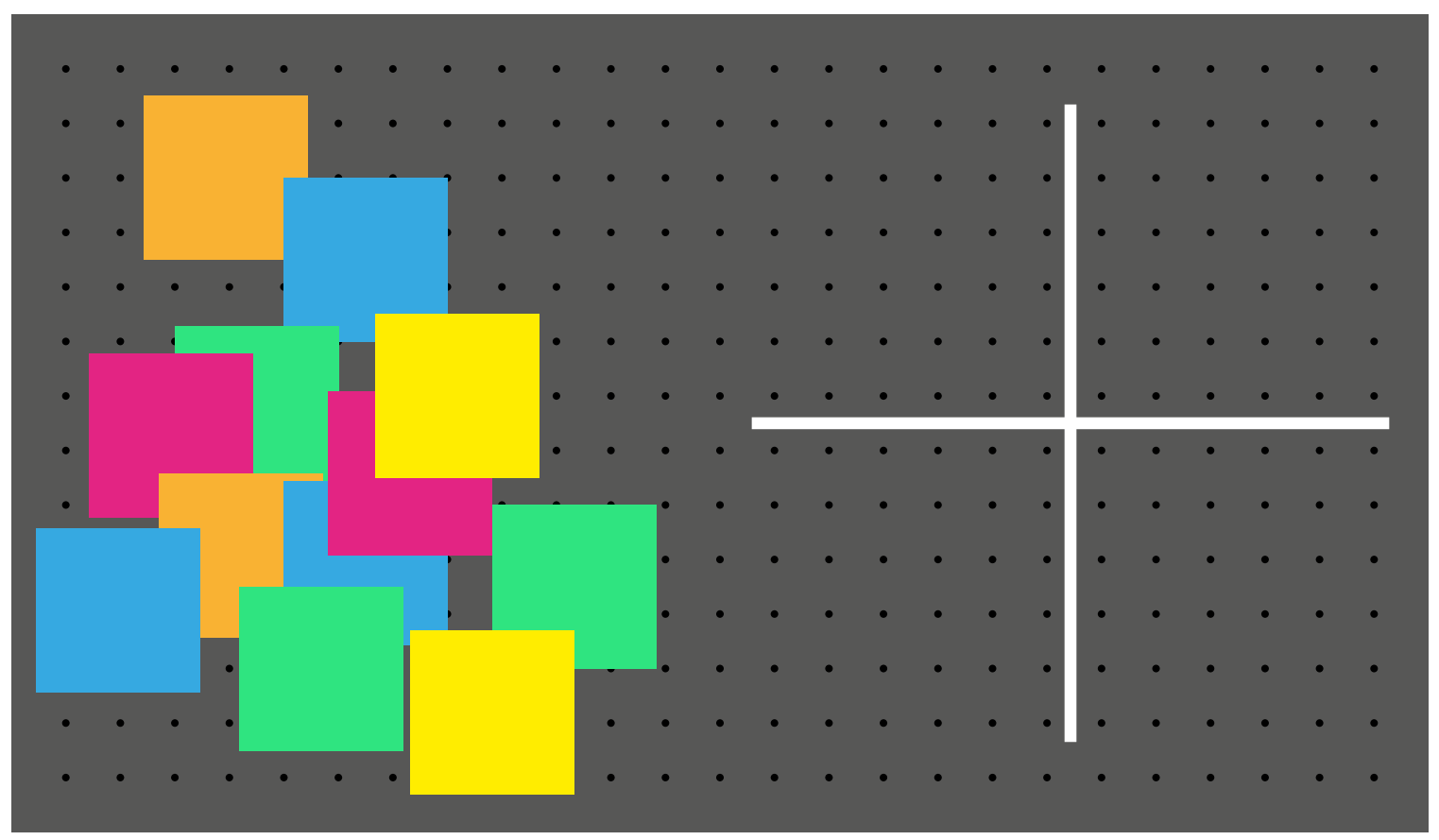

Figure 37: Digital board example of study. An example of what the digital board looked like when some of the groups started posting notes for the activity. 


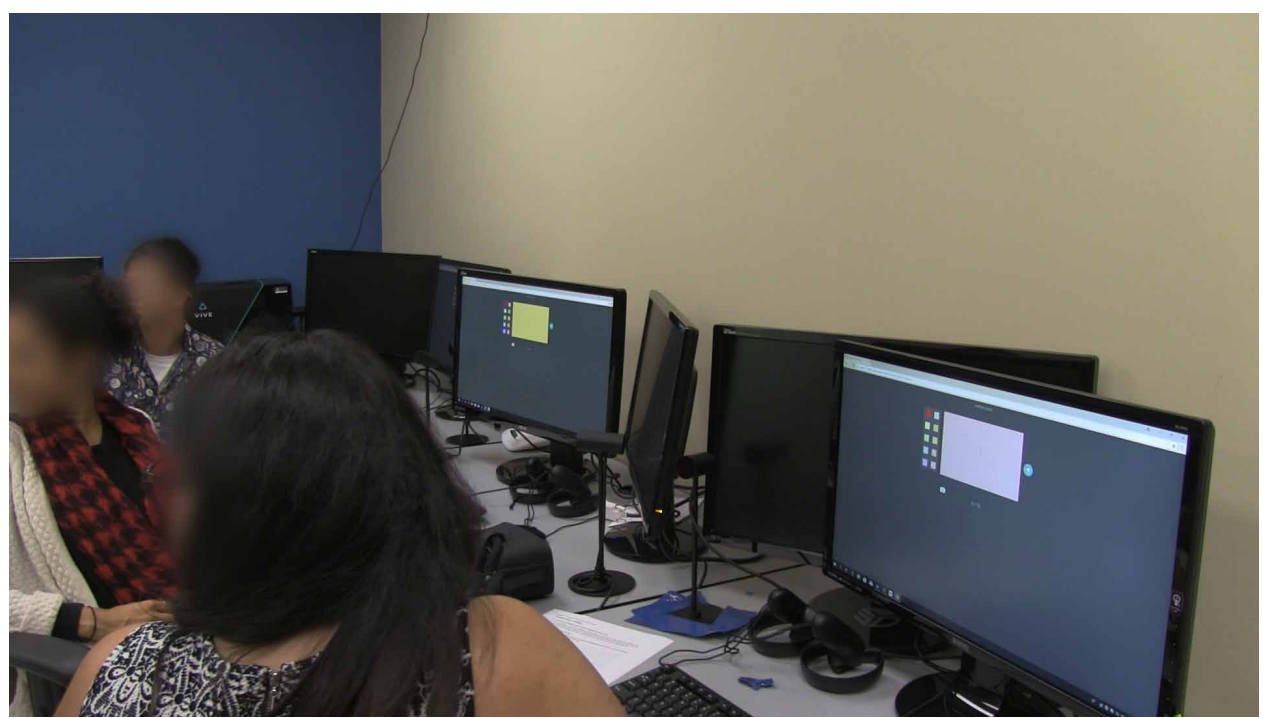

Figure 38: Participants during the digital medium. Participants turning around to look at the digital board after typing up a few notes on the client computers.

In VR there was no feedback to let participants know whether the others could see their writing or not. All the participants wrote but did not know if their note was seen by the others as they referenced it. Since the writing in VR was live, once participants notice each other writing for the first time it helps them understand how the program works and allows others to spectate easily, which all group members did between writing their own ideas.

\subsubsection{Insights from Participants' Actions}

Using Table 8 (below) to sort the insights by media for easier comparison, participants responded to others placing notes on the wall by first sticking their own notes up and then looking at what the others had written. Most of the participants paid attention when other members of the group wanted to move notes around and this usually led to some discussion about whether an ideas should stay where it was originally posted.

On the digital board, participants would look to see what was posted every once in a while, and after enough items were posted there was discussion to see where notes should be placed. Sometimes this led to more ideas being posted and repetitive thoughts grouped or deleted. As they used the board more and found more options and features, the participants became more interactive with the board. 
In the VR medium the participants would be on their own most of the time, occupying a spot on the VR boards. After writing down a few ideas they would step back to reread what they wrote to make changes and read what the others posted. If someone else was writing at the same time, the other participants would sometimes walk over and read what was being written. Just like it was a physical whiteboard where anyone can walk over to see.

\begin{tabular}{l|l|l} 
Physical & Digital & Virtual Reality \\
\hline - Sticking notes to the wall & $\begin{array}{l}\text { Turn around to look at the } \\
\text { board and see what others } \\
\text { - Looking at what the } \\
\text { others posted }\end{array}$ & $\begin{array}{l}\text { Spent most of their time } \\
\text { occupying one spot on the } \\
\text { board }\end{array}$ \\
$\begin{array}{ll}\text { - Moving things as a group } \\
\text { Discussion about }\end{array}$ & $\begin{array}{l}\text { Discussion after a few } \\
\text { potes appeared }\end{array}$ & $\begin{array}{l}\text { Participants would step } \\
\text { back to see the whole } \\
\text { board }\end{array}$ \\
& $\begin{array}{l}\text { Over time the participants } \\
\text { became more interactive } \\
\text { with the board }\end{array}$ & $\begin{array}{l}\text { Lots of looking over the } \\
\text { shoulder while writing }\end{array}$
\end{tabular}

Table 8: Summary of the insights from all media.

\subsubsection{What's Missing in Digital and VR}

The digital board per se was not necessarily missing anything yet the group interactions could have been smoother. The participants had to look over to the board which was behind them whenever they wanted to see what the others had posted. This often meant turning around one hundred eighty degrees, which looked uncomfortable. Most of the time they only turned their heads, which discouraged discussion while writing the notes. The position of the board was a limitation of the study since in a truly digital environment there would be no shared physical board, just a digital board on each person's screen or wall. A better method for the study to solve this could have been to rearrange the space to let the participants face the board while using the software to post the notes or using the program in a way that an overview of the whole board was shown to the participants at all times. This could allow group members to know when movement was 
happening at the board and help them keep track of when enough data was posted to start organizing. The board being easily visible means participants are not drawn away from the note making tools. This also means that any program that is limited on purpose to encourage interaction at the board should have the board in a central place so that all client device users can see the board clearly from where they are at their individual screens.

With the VR program, participants were not able to write notes on blocks and move them around; this encouraged re-writing notes if the note needed to be in a different place. This type of interaction was not time efficient and was made even worse when one person had to read aloud to another to write down. If a VR application is to be used in a design thinking environment it could benefit from basic text editing options like copy, paste and cut. The program did allow participants to move hands and fingers to visually describe concepts in VR, but none of the participants seemed to do this. They also did not use the medium for drawing or use any sketches to make it easier.

\subsubsection{Summary}

Feedback mechanisms during the physical medium like auditory (sounds of the participants working) and the visuals (notes stuck up and appearing on the wall) led participants to look at the board more during the first few steps as ideas were being created. During the digital medium participants tended to look at the board every few posts to see what the others had written and how many ideas had accumulated. This changed when it came to the VR medium as there was no auditory feedback, only visual feedback, the result of participants looking around every once in a while.

Insights from the participants throughout the study indicate that during the physical medium the activity had four main steps: sticking notes to the wall, looking at other posts, moving notes and discussion. In the digital medium, the steps were similar: look at other posts after sending their own, discussion and movement. During the VR medium participants spent most of their time occupying one spot at the board and would step back to see other posts. 
Missing interactions using the physical medium as a control include the smooth transition from desk to board. Rearranging the space would likely alleviate the problem and encourage more discussion. The ability to move existing text in the VR medium could increase discussion and let group members decide more easily where notes needed to be. Gesturing was also limited in the VR space and limbs such as the arms and hands were not used as much as in the other media.

\subsection{Main Research Question}

The main research question of this study is:

What are the interactions for collaboration and engagement among designers that are common in face-to-face experiences as well as $2 D$ and $3 D$ Virtual Environments?

This question focuses on the methods and interactions designed for physical and 2D spaces that were or could be used in 3D. The hypothesis that participants would try to transfer their own experiences from existing collaborations and design thinking sessions to new media was proven in some of the interactions, but not all. This change in media had some noticeable effects on how the participants completed the study and the results of the exercise.

\subsubsection{Adapting Interactions}

As technology advances and new kinds of media are adopted, people will need to adapt to new technologies. This can lead to the repurposing of old interactions or the creation of new ones, which can then spread to several objects and programs. To begin adapting to the new media in the study (2D and 3D), participants had to first figure out what was possible in VR. Two groups had the benefit of having participated in the other media of face-to-face and 2D before participating in the VR medium, which gave them some perspective on the kinds of interactions they would need to perform. The third group started the study with the VR medium so the did not receive that benefit. After figuring out the basic controls of the program they tried repeating what they did before. For the most part this worked out well, with all the participants writing down ideas and talking with the group, similar to using physical brainstorming methods. 
VR programs were already trying to take the basic interactions used in everyday life and implement them to good success (Frécon \& Nöu, 1998; Luxenburger \& Sonntag, 2016). The potential in these systems still leaves much room for improvement by identifying weak points in unique user interactions and filling in the missing pieces such as content management and text input.

\subsubsection{Design Opportunities}

In the context of this study, the interactions and gestures used were limited to creating (writing a new post-it note, typing a digital note and writing a note in VR) and moving (placing, removing, dragging, flicking) the ideas around. These interactions included grabbing a new post-it to write on, typing on a keyboard and waving hands in the air imitating writing on a board. In a 3D environment where participants are left to figure out how to navigate and collaborate on steps alone, it can be frustrating (Patel et al., 2006). Lack of experience and familiarity with new programs can lead to long learning times and slower results. Figures 25-30 show the VR medium taking longer than the physical and digital media. Opportunities for improvement involve adding flexibility to the VR environment so that participants have the freedom to move notes around, more like the physical medium. More input methods for text would also be an improvement as the current method for annotation was just writing by hand. This was shown to be a little difficult as the written text by many participants was not easy for them to write out and for others to read.

Additionally, there may be more interactions that are not yet ready for the transition from physical to VR. As users become more familiar with the actions that can be performed in VR, the case for more features and interactions can follow. This may also lead to a point where any further actions in VR become too much for users to handle and are better left for the physical realm. More exploration as the technology advances can lead to different challenges and possibilities for multi-user VR applications, such as the use of tracking objects and the use of sensors for feet and full body interactions. 
The higher distraction rate during the VR medium could be attributed to the slight anonymity of the program, since it was much harder to keep track of the others involved unless the participants were looking at each other's avatars. An opportunity to make the avatars less anonymous and have their activity available for others to check in with would make the system more suitable for management and supervision.

In summary, it was important for the participants to test out what could be accomplished in VR while using the existing interactions they already knew from brainstorming in a physical medium. The common features discovered for the main research question include giving users the freedom to move objects around like they can in reality, allowing flexibility for the input of text, and enabling some personalization through custom avatars. These insights can be considered as preliminary recommendations that may make it possible for VR to become a collaborative tool for design thinking activities, similar to those practised in this study. 


\section{Chapter 6. Conclusion}

Once a widespread literature review was conducted it became clear that research into design collaboration in 3D was sparsely available, yet tools for 2D formats existed in various arrangements. The topics of review included: observing the user experience, how behaviours differ in $2 \mathrm{D}$ vs $3 \mathrm{D}$, protocols for collaborating, communication, social interactions, and the nature of the environment or context.

The researcher investigation of research into VR interactions found that the majority of research focused only on single user experiences within a gaming context. A few companies are leaning toward business productivity as a new market, and with that multi-user VR experiences become an important factor, whether in co-located or remote environments. Some of the research also explored the different interactions that take place when multiple people are grouped for collaboration. While the physical world can be straight forward for most, digital media uses imitation for basic interactions to help ease users in. Unfortunately, not all features can be imitated, and this leads to unknown or inconsistent experiences.

Taking the constraints of the current consumer VR implementations (gaming) and the unexplored potential of VR-based design thinking collaboration into account, this research examined the issue from the perspective of design thinking and HCI.

Two methods of data collection were used to study the subject of this research. The first method involved observing participants while they completed a series of scenarios (exercises in different media). All of the exercises revolved around the concept of reducing food waste in the kitchen while preparing meals. The different media the participants used included a physical whiteboard with face-to-face interactions, a 2D white board with digital interactions, and a VR headset with 3D interactions. The second research method involved pre-test and post-test surveys to help triangulate the data analysis . The information gathered from the surveys indicate some background and context of the participants, such as experience with design thinking and their thoughts 
on VR as a useful design thinking and collaboration method. After the first two methods were completed, a further analysis took place, using the graphs that were based on observations made while tracking the activity, intensity and thus engagement of the participants. With the activity data available, comparisons between the scenarios and groups could take place, showing patterns in interaction and behaviour.

The results from observing the participants completing the steps provided insight into why some interactions occurred and how interaction aids in collaboration efforts while also showing gaps in the experience. For example, the flow of activity changed throughout the course of each exercise. In each scenario there were periods where each medium had strong and weak points which suggest possible opportunities for improvement. The analysis of this observation provided quantitative data that the researcher represented visually in the form of graphs and charts of the different interactions that took place during the scenarios. The results from the surveys indicated that the participants had lots of practice with design thinking methods, eliminating that as a variable of performance in exercise completion. This previous experience with design thinking also revealed any bias to the media and willingness to explore VR.

In conclusion, the results suggest that it is possible for collaborative design thinking sessions to be successfully completed in multiple forms of media. The interactions that occur were not always the same, but there seem to be enough basic gestures to reproduce similar results. Although currently, the dominant and most familiar forms of design thinking activities use physical brainstorming methods, with digital media following closely.

\subsection{Contributions}

The insights from this preliminary research may contribute to new awareness and recommendations for designing multi-user VR programs or collaborative remote environments specifically for design thinking activities. They provide insight into the nature of the interactions in the different commonly used media across design thinking 
activities that require tools for quickly writing ideas down using physical brainstorming methods, while taking advantage of the bigger space and image capabilities of the digital boards. These insights may also evolve to creating new methods for design thinking that are tailored to the benefits that features for conducting design thinking exercises in VR can offer such as higher engagement through active participation. Drawing from the research questions, the contributions of this research include the following insights:

\section{Media Choice}

- Flexibility of text input is a large part of the VR experience. Allowing users to find the most comfortable and efficient way to enter text could improve warmup periods.

- While VR is an interesting medium to take advantage of for collaborative activities such as design thinking, there is still an appreciation for traditional methods like post-it notes, and both methods can co-exist and build on the advantages of each.

- Users unfamiliar with VR may find the medium very intriguing and are more engaged when trying something new. Personal avatars may help users become more invested in a program or system.

- There is a need for both careful instructions when introducing users to VR and the use of a warm-up period to help users learn functions. This warm-up period is very important as it creates the first impression on users new to VR. User's unfamiliar or having trouble with other media can also benefit from a warm-up as not everyone will be able to learn programs like Nureva's Span as quickly.

- Designers when given the option, may prefer one medium over the others.

\section{Interactions}

- Users are comfortable having the freedom to move objects. In the VR medium tested, not everything could be moved. This meant if text was written in one spot, it stayed in that spot unless rewritten or erased. 
- The cues from the environment could help the group stay on task and prevent individuals from falling behind. These cues may take the form of auditory or visual feedback such as alerts in the corner of a screen or vibration that can guide users.

- Interactions that occurred during the study include interactions with the media and interactions with other participants. Sometimes these interactions include both.

- Verbal communication is always used and seems to not be affected by the media used for the collaborative activity.

- During a design thinking activity, delegation often takes place to divide and conquer so that problems can be more easily solved in chunks. This was present in the physical, digital and VR media, especially since the tools used facilitated individual work as well as working in a group.

\subsection{Limitations}

For the value of future research and for some context into why certain exercises and media were used in this study it is important to mention the limitations of this research.

The main limitation of the study was the sample size and selection used. With a small sample size of only nine participants spread across three groups, the results from this study do not necessarily represent the designer population as a whole. As a result, this study should be considered as a preliminary pilot study for a more extensive investigation across the three specific media. In addition, since the participants were designers with experience in design thinking, the results cannot be applied to the general population.

Another limitation of the study was that some of the uncommon interactions that did not occur in some groups may have been discovered if the participants had been given more time to explore the different media. Group 2 especially was very quick with completing the exercises while Group 1 went overtime in each exercise. There was a small acclimation period where participants could learn how to use some of the technology in the study. It was unclear if this period of time was increased whether 
more unique collaborative interactions would have occurred. This also includes the use of moving notes and ideas. While post-it notes could be moved around in the physical medium and digital notes could be dragged anywhere on the virtual board, in VR once something was written anywhere it remains in that position indefinitely. This created a disadvantage in the VR medium when looking at the codex entries influencing how much activity occurred.

Verbal exchange during the exercises was not impacted by limitations in any of the media, since the participants were all located within the same room where they could speak freely to each other without having to go through any additional setup or apparatus. If any of the exercises were conducted in a way that took advantage of the different media, such as remote collaboration, verbal exchange would be affected and the outcome may have been vastly different. Since remote collaboration is a key feature of digital design thinking exercises, this study was unable to capture or reconstruct the exact features of the digital medium. However, this leaves room for future research into the remote factors that affect collaboration in VR.

Being co-located also had its limitations when it came to the cues during the digital medium. Throughout the first few steps of each group the only indication that other participants were posting notes was the clacking of keyboards as participants would type out quick bursts and pause in between. This auditory feedback would let the other participants gauge how fast and how many notes their group members were posting and would not necessarily be available if any of the participants had been working remotely. This highlights the importance of multi-modal feedback cues in terms of the activities of the other team members when working in digital and VR media.

Lastly, although the problem and instructions for solving it was given to the groups, it was meant as a mock exercise so that the participants would go through design thinking brainstorming activities. It was possible that the context of the problem may have influenced how the participants worked together. Depending on their preferences 
for cooking and possible eating habits, previous experiences might have had an effect on how open toward each other the participants were.

\subsection{Future Research}

As noted in this study, the findings, contributions and limitations offer insights as a preliminary pilot study into collaborative interactions for designers to consider in developing VR tools for design thinking brainstorming activities. Further research could expand upon the contributions of this study. The design research opportunities identified here offer topics that can lead to future research such as:

- How performance between participants would differ in remote or co-located environments using digital or VR collaboration.

- Would the use of a VR system that supports more sensors and trackers engage the participants more?

- Does a VR collaboration system work better for smaller or larger groups?

Additional areas for possible future research include addressing some of the limitations encountered during this study. There is room for researchers to replicate the study using larger sample sizes or even using a different exercise than food waste reduction. This study was purposely limited to designers in a collaborative design thinking experience, but future research might also choose a group of participants with a variety of backgrounds as well as a different activity to get a better representation of the general population as a whole.

The results of this design research come at a time when virtual reality has become very accessible to consumers, in particular gamers, but in professional environments it still needs to find its value. As the design community continually adopts technology for creative uses, professionals are learning to work in a more collaborative and interactive way using remote communication tools. This rapidly evolving space offers exciting opportunities for design researchers to help conceptualize products that, creatively and rationally, meet the needs of design thinkers everywhere. 


\section{Bibliography}

Ã, C. A., \& Argelaguet, F. (2007). Anisomorphic ray-casting manipulation for interacting with 2D GUIs, 31, 15-25. https://doi.org/10.1016/j.cag.2006.09.003

Bae, S., Lee, H., Park, H., Cho, H., Park, J., \& Kim, J. (2012). The effects of egocentric and allocentric representations on presence and perceived realism: Tested in stereoscopic 3D games. Interacting with Computers, 24(4), 251-264. https://doi. org/10.1016/j.intcom.2012.04.009

Bianchi, D. A. da C. (2015). Exploring Fictional Scenarios Of Pervasive Computing To Identify Meaningful Gestures For Intuitive Interactions. Carleton University.

Billinghurst, M. (2002). Shared space: Explorations in collaborative augmented reality, 345.

Brown, T. (2008). Design thinking. Harvard Business Review.

Brown, T. (2009). Change by Design: How Design Thinking Transforms Organizations and Inspires Innovation. Director 00123242. https://doi.org/10.1017/ CBO9781107415324.004

Charness, G., Gneezy, U., \& Kuhn, M. A. (2012). Experimental methods: Betweensubject and within-subject design. Journal of Economic Behavior and Organization. https://doi.org/10.1016/j.jebo.2011.08.009

Chellali, A., Milleville-Pennel, I., \& Dumas, C. (2008). Elaboration of a common frame of reference in collaborative virtual environments. Proceedings of the 15th European Conference on Cognitive Ergonomics: The Ergonomics of Cool Interaction, 21:1-21:8. https://doi.org/10.1145/1473018.1473045

Cozby, P. C., \& Bates, S. C. (2015). Methods in Behavioral Research (12th Edition). Behaviour Research and Therapy. https://doi.org/10.1016/0005-7967(79)90091-3 
David Lavenda. (2014). How The Post-it Note Could Become The Latest Innovation Technology, 10-13. Retrieved from https:/www.fastcompany.com/3027722/howthe-post-it-note-could-become-the-latest-innovation-technology

Dell Visor. (2018). Retrieved January 3, 2018, from http://www.dell.com/en-ca/shop/dellvisor/apd/536-bbbr/gaming

Frécon, E., \& Nöu, A. A. (1998). Building Distributed Virtual Environments to Support Collaborative Work. Proc. of the ACM Symp. on Virtual Reality Software and Technology, 105-113. https://doi.org/10.1145/293701.293715

Gauglitz, S., Nuernberger, B., Turk, M., \& Höllerer, T. (2014). In touch with the remote world. Proceedings of the 20th ACM Symposium on Virtual Reality Software and Technology - VRST '14, 197-205. https://doi.org/10.1145/2671015.2671016

Glencross, M., Chalmers, A. G., Lin, M. C., Otaduy, M. A., \& Gutierrez, D. (2006). Exploiting perception in high-fidelity virtual environments. ACM SIGGRAPH 2006 Courses on - SIGGRAPH '06, 1. https://doi.org/10.1145/1185657.1185814

Goodwin, C. J. (2010). Research Methods in Psychology: Methods and design. John Wiley \& Sons, Inc. https://doi.org/10.1002/15213773(20010316)40:6<9823::AID-ANIE9823>3.3.CO;2-C

Heldal, I., Spante, M., \& Connell, M. (2006). Are two heads better than one?: Objectfocused work in physical and in virtual environments. Proceedings of the ACM Symposium on Virtual Reality Software and Technology, VRST, 287-296. https:// doi.org/10.1145/1180495.1180553

HTC Vive Controller. (2018). Retrieved January 3, 2018, from https://www.vive.com/eu/ accessory/controller/

IBM. (2017a). Scenario Map, 1-5.

IBM, D. (2017b). Feedback Grid. Retrieved November 21, 2017, from https://www.ibm. com/design/thinking/activities/feedback-grid 
IBM, D. (2017c). Moodboarding. Retrieved November 21, 2017, from https://www.ibm. com/design/thinking/activities/moodboard

IBM, D. (2018). Enterprise Design Thinking Field Guide.

Kalbach, J. (2016). Mapping Experiences. O’Reilly.

Kolko, J. (2011). Thoughts on Interaction Design: Second Edition. Thoughts on Interaction Design: Second Edition. https://doi.org/10.1016/C2009-0-61348-9

Kolko, J. (2014). Well Designed. Boston.

Kuhnke, E. (2012). Body Language For Dummies (Second). For Dummies.

Kuniavsky, M. (2003). Observing the User Experience: A Practitioner's Guide to User Research. Observing the User Experience: A Practitioner's Guide to User Research. https://doi.org/10.1016/B978-1-55860-923-5.X5026-8

LaViola Jr., J. J. (2017). 3D User Interfaces.

Lloyd, D., Benford, S., \& Greenhalgh, C. (1999). Formations: explicit group support in collaborative virtual environments. ... of the ACM Symposium on Virtual ..., 162-163. Retrieved from http://dl.acm.org/citation.cfm?id=323690

Luxenburger, A., \& Sonntag, D. (2016). MedicalVR : Towards Medical Remote Collaboration Using Virtual Reality, 321-324. https://doi. org/10.1145/2968219.2971392

Martin, B., \& Hanington, B. (2012). Universal Methods of Design. Rockport Publishers. Mason, A. H. (2001). Sensory Information for Collaborative Interaction in Augmented Environments. CHI '01 Extended Abstracts on Human Factors in Computing Systems, (Figure 1), 77-78. https://doi.org/10.1145/634067.634116

Miller, J. K. (2010). Intercultural interaction and collaboration in a virtual world, 171174. https://doi.org/10.1145/1841853.1841879

Mural. (2018). Mural. Retrieved January 13, 2018, from mural.co 
Musse, S. R., Babski, C., Capin, T., \& Thalmann, D. (1998). Crowd modelling in collaborative virtual environments. Proceedings of the ACM Symposium on Virtual Reality Software and Technology 1998 - VRST '98, 115-123. https://doi. org/10.1145/293701.293716

Nureva. (2018). Span. Retrieved October 17, 2017, from nureva.com

Oculus. (2018). Oculus Rift Accessories. Retrieved January 3, 2018, from https://www. oculus.com/accessories/

Ovsyannykov, I. (2016). Mood Boards: Why and How To Create Them. Retrieved March 1, 2018, from https://creativemarket.com/blog/mood-boards-why-and-how-tocreate-them

Patel, H., Stefani, O., Sharples, S., Hoffmann, H., Karaseitanidis, I., \& Amditis, A. (2006). Human centred design of 3-D interaction devices to control virtual environments. International Journal of Human Computer Studies, 64(3), 207-220. https://doi.org/10.1016/j.ijhcs.2005.08.010

Pxhere. (2019). Business People in a Meeting. Retrieved from https://pxhere.com/en/ photo/1435997

Ro, H., Chae, S., Kim, I., Byun, J., Yang, Y., Park, Y., \& Han, T. (2017). A Dynamic Depth-Variable Ray-Casting Interface for Object Manipulation in AR Environments, 5-10.

Saldaña, J. (2015). The coding manual for qualitative researchers. Sage. https://doi. org/10.1017/CBO9781107415324.004

Salimian, M. H. (2015). Exploring group awareness in a mixed reality collaborative environment. Proceedings of the 2015 ACM International Joint Conference on Pervasive and Ubiquitous Computing and Proceedings of the 2015 ACM International Symposium on Wearable Computers - UbiComp '15, 495-501. https://doi.org/10.1145/2800835.2801654 
Salimian, M., Reilly, D., Brooks, S., Mackay, B., \& Scotia, N. (2016). Physical-Digital Privacy for Mixed Reality Collaboration: An Exploratory Study. Iss '16, 261-270. https://doi.org/10.1145/2992154.2992167

Schwartz, P., Yen, S., Bricker, L., Campbell, B., Furness, T., Inkpen, K., ... Tanney, S. (1998). Virtual playground. Proceedings of the ACM Symposium on Virtual Reality Software and Technology 1998 - VRST '98, 43-50. https://doi. org/10.1145/293701.293707

Spence, C., \& Gallace, A. (2011). Multisensory design: Reaching out to touch the consumer. Psychology and Marketing, 28(3), 267-308. https://doi.org/10.1002/ mar.20392

Swiftkey. (2019). Swiftkey Keyboard. Retrieved from https://lh3.googleusercontent. com/5xWwHb6ACFKZBRgrBU1G2cKfwNQ4KDU8BmM8CtZhDb_ XR6AjFaz_gvQPb_nJQ21Bxg=w1720-h1020

Swing, E. (2000). Adding immersion to collaborative tools. Proceedings of the Fifth Symposium on Virtual Reality Modeling Language (Web3D-VRML) - VRML '00, 63-68. https://doi.org/10.1145/330160.330178

Tiwari, A., \& Holtham, C. (1998). Learning groupware through using groupware Computer supported collaborative learning with face-to-face students. ITiCSE '98, 236-238. https://doi.org/10.1145/282991.283553

Unity. (2018). User Interfaces for VR. Retrieved January 4, 2018, from https://unity3d. com/learn/tutorials/topics/virtual-reality/user-interfaces-vr 


\section{Glossary}

2D (two- $\quad$ A working space that exists in only two dimensions rather than three dimensional) and usually refers to a digital space. I.e. web graphics and posters. 3D (three- $\quad$ A working space that exists in 3 dimensions, typically used when dimensional) describing a digital space. I.e. most VR and modern video games use $3 \mathrm{D}$ environments rather than $2 \mathrm{D}$.

Affinity Diagram A tool that gathers large amounts of language data (ideas, opinions, issues) and organizes the data into groupings based on their natural relationships.

API (application

A set of functions and procedures that allow the creation of programming applications that access the features or data of an operating system, interface) another application, or other service (google.com).

Clustering Grouping together ideas and thoughts that are similar, to be counted as one general thought.

Collaboration The act of a group of people work together to accomplish a common goal.

Engagement The level of interest and involvement a person has/demonstrates in the current activity.

Feedback Grid An activity to help gather and organize any sort of feedback and to then unpack questions and ideas - either in real time or after-the-fact - as an efficient means of determining next steps (IBM, 2017b).

Latency (or lag) The delay between when an action or movement is performed, and a response is shown on screen, usually measured in milliseconds.

Manipulation $\quad$ Modifying an object through translation, rotation or scaling. Can also include other properties like colour, texture and lighting within a digital context. 
Moodboard A composition of a collage of images, text, and samples of objects that can be used to communicate an aesthetic or idea.

Mural

Navigation

Nureva

Playback

Ray casting

Selection

Text input

Face-to-face

Virtual environment (VE)

Virtual hands

Virtual reality (VR)
Digital workspace software for visual collaboration, inspiration and innovation anytime, anywhere, on any device (Mural.co, 2019).

Consists of two subtasks, one of which is travel; the task of moving from the current location to a new target location and the other being wayfinding (cognitive route planning). (LaViola Jr., 2017).

Company that designs and builds audio and visual collaboration tools that make it possible for teams to collaborate remotely (Nureva. com, 2019).

When people orally summarize and describe what they just learned to present the main points to team members. (IBM, 2017b).

A method of pointing to objects in a virtual reality environment for remote selection/manipulation.

Specifying (choosing) an object as a point of interest for subsequent actions, such as manipulation in digital and virtual environments. A way to add text to the object/point of interest in digital or virtual media. When two or more people are in close proximity speaking or working together.

A synthetic, spatial (usually 3D) world seen from a first-person point of view. The view in a virtual environment is under the real-time control of the user (LaViola Jr., 2017).

A virtual representation of a user's hands.

An approach that uses displays, tracking, and other technologies to immerse the user in a VE (LaViola Jr., 2017). 
APPENDIX A

TCPS2 Certificate 


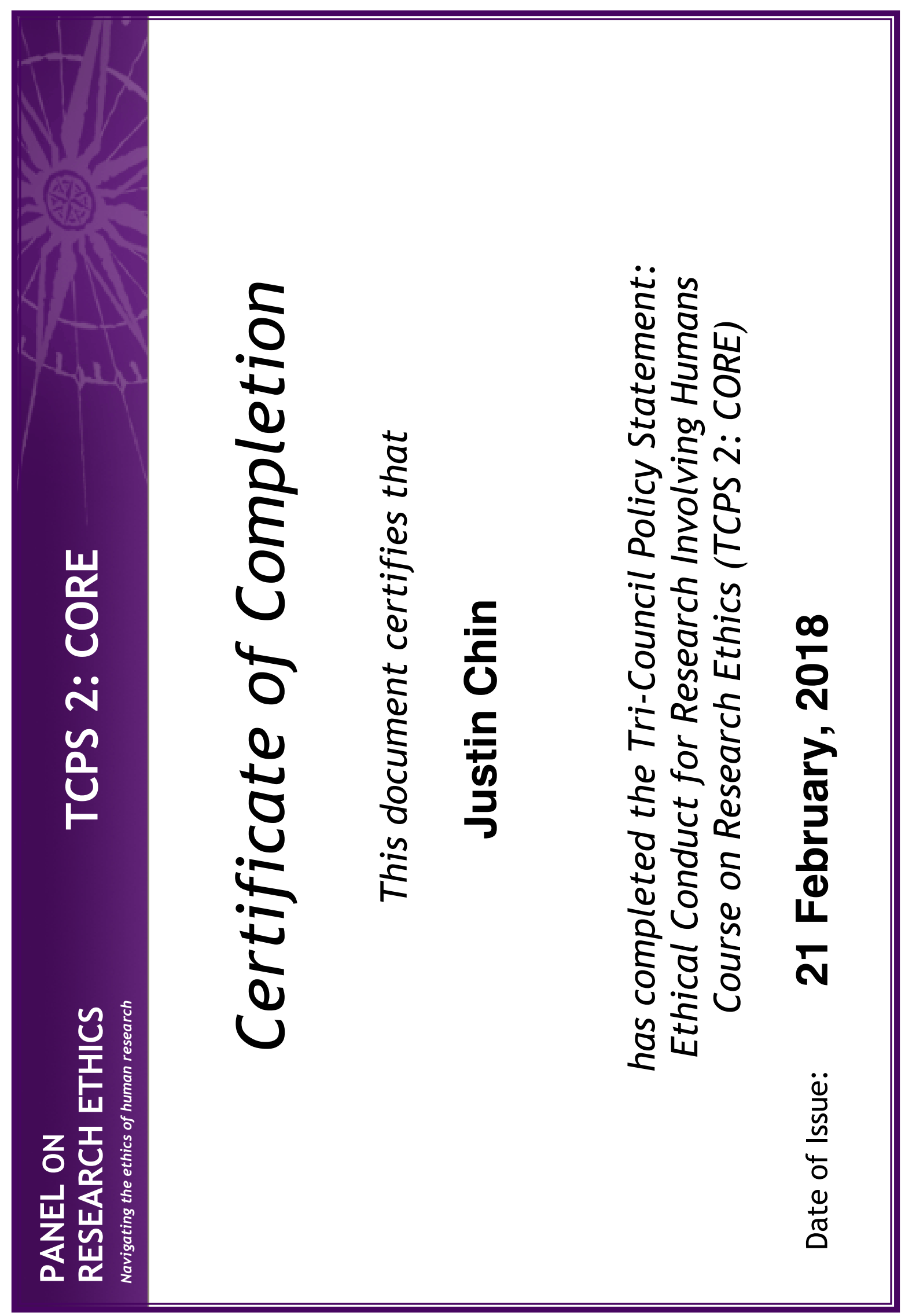




\section{APPENDIX B}

Experimental Scripts for each Group 


\section{Study Consent Process Script}

Consent forms must be completed before the activity is started. (7 $\mathrm{min})$

Thank you for agreeing to participate. We are very interested to learn about your perspective on design thinking and the interactions that it involves.

- The purpose of this study is to observe and record the different interactions that take place when designers collaborate in different media.

- The information you give us is completely confidential, and we will not associate your name with anything you say in this study/activity.

- We would like to record the activity so that we can make sure to capture your interactions and comments we see and hear during this session. No names will be attached to the content and the recordings will be destroyed as soon as they are transcribed, or any relevant excerpt saved.

- You may refuse to answer any question or withdraw from the study during the exercise, and up until 2 weeks after the exercise.

- We understand how important it can be that this information is kept private and confidential and offer the choice to be anonymous or not. We will ask that everyone in this room protect this information.

- The design activity consists of 3 parts where you will be given different materials to complete each task. In no specific order, you will be completing the tasks using post-it notes for one, a computer and digital board for another and a virtual reality headset for the remaining one. There is a possible risk of nausea if you have no experience with virtual reality. Please do not participate if you are susceptible to nausea.

\section{Experiment group 1: starts with the physical post-it notes, then virtual reality and lastly with $2 \mathrm{D}$ digital boards. Total time is $\mathbf{2 5}$ min.}

1.1. (5min) Welcome participants, introduction of myself and the note taker. We will review the following:

2.1. Who we are and what we're trying to do.

2.2. What will be done with this information.

2.3. Why we asked you to participate.

During this time, we will also give out the pre-test survey for the participants to complete.

1.2. Explanation of the process

2.1. We learn from you (the participant) both positive and negative.

2.2. In the case of the activity group, we ask that you follow the instructions on the task.

2.3. No virtue in long lists: we're looking for specific information.

2.4. Explain how long each section will last. 
2.5. Feel free to move around during the activity.

2.6. Where are the bathrooms? Exit?

2.7. Help yourself to refreshments.

1.3. Ground rules for the activity.

2.1. Everyone should participate.

2.2. Information provided in the activity must be kept confidential.

2.3. Stay with the group discussion and please don't have side conversations.

2.4. Turn off cell phones is possible or at least keep them away.

2.5. Have fun.

1.4. Turn on recording equipment.

1.5. Ask the group if there are any questions before we get started and address those questions before moving on.

1.6. Introductions of participants to each other. Name and field of work.

1.7. (5 min) Begin first section of the as-is scenario map activity

2.1. Explain that this activity is about placing yourself in the given task.

2.2. As-is scenario maps help document a team's collective understanding of existing user workflows and are best used as precursors to exploring new ideas. When creating an as-is scenario map, be honest and thorough. It's important to articulate your user's actual current experience. Don't neglect tasks or qualities that are not ideal or positive. When you've completed the map, check it over

2.3. Background information is given to focus the topic:

\subsubsection{You are asked to imagine that you have decided to reduce waste for} one month in the kitchen when preparing a meal. Participants will use the provided materials to capture ideas and post them to the white board. An example will be shown so they have a good idea of what to do starting with mapping what they think the current user experience is like.

1.8. (5 min) After a few notes have been posted, the participants can start to annotate the unknowns for later inquiry or validation. As a group try to fill in those unknowns and answer any remaining questions together.

1.9. (5 $\mathrm{min}$ ) The second section will be analyzing the current map to identify what the positives and negatives of the current user experience are. As a group they will need to make this decision and are free to move things around as needed.

1.10. (5 min) Focusing on the negatives now, the group will try to come up with solutions to try and reduce the impact they have on the user experience. Ex. If finding a clean place to sit is a major problem, what can be done to solve this?

1.11. The last step will be presenting what the group found about the user 
experience to the note taker and I.

1.12. Participants are thanked for their time and given a small break before the next exercise.

Materials needed include post it notes, a large whiteboard and markers.

Total time is $30 \mathrm{~min}$.

1.13. (5 min) Participants are guided to the computers and VR equipment that the activity will take place on.

2.1. A short introduction to the program and how to do certain functions is given.

2.2. Give the participants a few minutes to familiarize themselves with the program.

1.14. Ask the group if there are any questions before we get started and address those questions before moving on.

1.15. Turn on recording equipment.

1.16. (5 min) Begin first section of the to-be scenario map activity

2.1. Explain that this activity is about creating an ideal scenario for themselves working towards the goal of waste reduction during meal preparation. This ideal scenario is based off what was found in the first exercise.

2.2. To-be scenario maps tell the story of a desired future. Any solutions presented in a to-be scenario should ideally correlate to pain points identified in the as-is.

2.3. Participants will use the provided equipment to capture ideas and post them to the virtual white boards. An example will be shown so they have a good idea of what to do starting with mapping what they think is the current user experience.

1.17. (10 min) Participants will try to fill the virtual board with notes as they go through the user experience.

1.18. (5 min) Cluster similar ideas and discuss. Search for patterns and themes.

1.19. (5 $\mathrm{min})$ The last step will be presenting the new user experience to the note taker and I, explaining how their solution solved the problems from the first exercise.

1.20. Participants are thanked for their time and given a small break before the next exercise.

Materials needed include multiple VR sets in a room big enough to house all of them running at the same time.

Total time is $25 \mathrm{~min}$ 
1.21. (5 min) Participants are guided to the computers that the activity will take place on.

2.1. A short introduction to the program and how to do certain functions is given.

1.22. Ask the group if there are any questions before we get started and address those questions before moving on.

1.23. Turn on recording equipment.

1.24. Begin first section of the Feedback Grid activity

2.1. Explain that this activity is about gathering and organizing feedback from the other activities to keep building deeper. A quadrant map presented will have the following labels: things that worked, things to change, questions we still have, and new ideas to try.

2.2. Participants will use the provided equipment to fill in each quadrant with notes that are specific and give criticism.

1.25. (5 min) Participants will try to fill the quadrant map with digital notes.

1.26. (5 min) Cluster similar ideas and discuss. Search for patterns and themes.

1.27. (5 min) The last step will be presenting the patterns and themes that they found to the note taker and I.

1.28. (5 min) The participants are given a post-test survey to complete (Appendix D).

1.29. Participants are thanked for their time and shown the way out.

Materials needed include a large format display, multiple devices and software to emulate the sticky notes. Participants could possibly run a web version on their own devices.

\section{Experiment group 2: starts with 2D digital boards, then physical post it notes and lastly with the virtual reality boards. Time is $30 \mathrm{~min}$.}

1.1. (5 min) Welcome participants, introduction of myself and the note taker. We will review the following:

2.1. Who we are and what we're trying to do.

2.2. What will be done with this information.

2.3. Why we asked you to participate.

During this time, we will also give out the pre-test survey for the participants to complete.

1.2. Explanation of the process

2.1. We learn from you (the participant) both positive and negative. 
2.2. In the case of the activity group, we ask that you follow the instructions on the task.

2.3. No virtue in long lists: we're looking for specific information.

2.4. Explain how long each section will last.

2.5. Feel free to move around during the activity.

2.6. Where are the bathrooms? Exit?

2.7. Help yourself to refreshments.

1.3. Ground rules for the activity.

2.1. Everyone should participate.

2.2. Information provided in the activity must be kept confidential.

2.3. Stay with the group discussion and please don't have side conversations.

2.4. Turn off cell phones is possible or at least keep them away.

2.5. Have fun.

1.4. (5 min) Participants are guided to the computers that the activity will take place on.

1.5. A short introduction to the program and how to do certain functions is given.

1.6. Turn on recording equipment.

1.7. Ask the group if there are any questions before we get started and address those questions before moving on.

1.8. Introductions of participants to each other. Name and field of work.

1.9. Begin first section of the as-is scenario map activity

2.1. Explain that this activity is about placing yourself in the given task.

2.2. As-is scenario maps help document a team's collective understanding of existing user workflows and are best used as precursors to exploring new ideas. When creating an as-is scenario map, be honest and thorough. It's important to articulate your user's actual current experience. Don't neglect tasks or qualities that are not ideal or positive. When you've completed the map, check it over

2.3. Background information is given to focus the topic:

3.3.1. You are asked to imagine that you have decided to reduce waste for one month in the kitchen when preparing a meal. Participants will use the provided equipment to capture ideas and post them to the digital white board. An example will be shown so they have a good idea of what to do starting with mapping what they think the current user experience is like.

1.10. (5 min) After a few notes have been digital posted to the large display, the participants can start to annotate the unknowns for later inquiry or validation. As a group try to fill in those unknowns and answer any remaining questions together.

1.11. (5 $\mathrm{min}$ ) The second section will be analyzing the current map to identify what the positives and negatives of the current user experience are. As a group they will need to make 
this decision and are free to move things around as needed.

1.12. (5 min) Focusing on the negatives now, the group will try to come up with solutions to try and reduce the impact they have on the user experience. Ex. If finding a clean place to sit is a major problem, what can be done to solve this?

1.13. (5 min) The last step will be presenting what the group found about the user experience to the note taker and I.

1.14. Participants are thanked for their time and given a small break before the next exercise.

Materials needed include a large format display, multiple devices and software to emulate the sticky notes. Participants could possibly run a web version on their own devices.

Time is $20 \mathrm{~min}$

1.15. (5 min) Participants are guided to a room with materials on the tables to perform the physical post-it note section of the activity.

1.16. Turn on recording equipment.

1.17. Ask the group if there are any questions before we get started and address those questions before moving on.

1.18. Begin first section of the to-be scenario map activity

2.1. Explain that this activity is about creating an ideal scenario for themselves working towards the goal of waste reduction during meal preparation. This ideal scenario is based off what was found in the first exercise.

2.2. To-be scenario maps tell the story of a desired future. Any solutions presented in a to-be scenario should ideally correlate to pain points identified in the as-is.

2.3. Participants will use the provided materials to capture ideas and post them to the white board. An example will be shown so they have a good idea of what to do starting with mapping what they think is the current user experience should be.

1.19. (5 min) Participants will try to fill the map with post-it notes.

1.20. (5 min) Cluster similar ideas and discuss. Search for patterns and themes.

1.21. (5 min) The last step will be presenting the new user experience to the note taker and I explaining how their solution solved the problems from the first exercise.

1.22. Participants are thanked for their time and given a small break before the next exercise.

Materials needed include post it notes, a large whiteboard and markers. 
Time is $25 \mathrm{~min}$

1.23. (10 min) Participants are guided to the computers and VR equipment that the activity will take place on.

2.1. A short introduction to the program and how to do certain functions is given.

2.2. Give the participants a few minutes to familiarize themselves with the program.

1.24. Turn on recording equipment.

1.25. Ask the group if there are any questions before we get started and address those questions before moving on.

1.26. (15 min) Begin first section of the Feedback Grid activity

2.1. Explain that this activity is about gathering and organizing feedback from the other activities to keep building deeper. A quadrant map presented will have the following labels: things that worked, things to change, questions we still have, and new ideas to try.

2.2. Participants will use the provided equipment to fill in each quadrant with notes that are specific and give criticism.

2.3. Cluster similar ideas and discuss. Search for patterns and themes.

1.27. The last step will be presenting the patterns and themes that they found to the note taker and $\mathrm{I}$.

1.28. The participants are given a post-test survey to complete (Appendix D).

1.29. Participants are thanked for their time and shown the way out.

Materials needed include a large format display, multiple devices and software to emulate the sticky notes. Participants could possibly run a web version on their own devices.

\section{Experiment group 3: starts with the virtual reality activity, then physical post it notes, and lastly the 2D digital activity. Time is $35 \mathrm{~min}$}

1.1. (5 min) Welcome participants, introduction of myself and the note taker. We will review the following:

2.1. Who we are and what we're trying to do.

2.2. What will be done with this information.

2.3. Why we asked you to participate.

During this time, we will also give out the pre-test survey for the participants to complete.

1.2. Explanation of the process

2.1. We learn from you (the participant) both positive and negative. 
2.2. In the case of the activity group, we ask that you follow the instructions on the task.

2.3. No virtue in long lists: we're looking for specific information.

2.4. Explain how long each section will last.

2.5. Feel free to move around during the activity.

2.6. Where are the bathrooms? Exit?

2.7. Help yourself to refreshments.

1.3. Ground rules for the activity.

2.1. Everyone should participate.

2.2. Information provided in the activity must be kept confidential.

2.3. Stay with the group discussion and please don't have side conversations.

2.4. Turn off cell phones is possible or at least keep them away.

2.5. Have fun.

1.4. (5 min) Participants are guided to the computers and VR equipment that the activity will take place on.

2.1. A short introduction to the program and how to do certain functions is given.

2.2. Give the participants a few minutes to familiarize themselves with the program.

1.5. Turn on recording equipment.

1.6. Ask the group if there are any questions before we get started and address those questions before moving on.

1.7. Introductions of participants to each other. Name and field of work.

1.8. (5 min) Begin first section of the as-is scenario map activity

2.1. Explain that this activity is about placing yourself in the given task.

2.2. As-is scenario maps help document a team's collective understanding of existing user workflows and are best used as precursors to exploring new ideas. When creating an as-is scenario map, be honest and thorough. It's important to articulate your user's actual current experience. Don't neglect tasks or qualities that are not ideal or positive. When you've completed the map, check it over

2.3. Background information is given to focus the topic:

\subsubsection{You are asked to imagine that you have decided to reduce waste for} one month in the kitchen when preparing a meal. Participants will use the provided equipment to capture ideas and post them to the digital white board. An example will be shown so they have a good idea of what to do starting with mapping what they think the current user experience is like.

1.9. (5 min) After a few notes have been virtually posted to the virtual reality environment, the participants can start to annotate the unknowns for later inquiry 
or validation. As a group try to fill in those unknowns and answer any remaining questions together.

1.10. (5 min) The second section will be analyzing the current map to identify what the positives and negatives of the current user experience are. As a group they will need to make this decision and are free to move things around as needed.

1.11. (5 min) Focusing on the negatives now, the group will try to come up with solutions to try and reduce the impact they have on the user experience. Ex. If finding a clean place to sit is a major problem, what can be done to solve this?

1.12. The last step will be presenting what the group found about the user experience to the note taker and I.

1.13. Participants are thanked for their time and given a small break before the next exercise.

Materials needed include a large format display, multiple devices and software to emulate the sticky notes. Participants could possibly run a web version on their own devices.

Time is $20 \mathrm{~min}$

1.14. (5 min) Participants are guided to a room with materials on the tables to perform the physical post-it note section of the activity.

1.15. Turn on recording equipment.

1.16. Ask the group if there are any questions before we get started and address those questions before moving on.

1.17. Begin first section of the to-be scenario map activity

2.1. Explain that this activity is about creating an ideal scenario for themselves working towards the goal of waste reduction during meal preparation. This ideal scenario is based off what was found in the first exercise.

2.2. To-be scenario maps tell the story of a desired future. Any solutions presented in a to-be scenario should ideally correlate to pain points identified in the as-is.

2.3. Participants will use the provided materials to capture ideas and post them to the white board. An example will be shown so they have a good idea of what to do starting with mapping what they think is the current user experience should be.

1.18. (5 $\mathrm{min})$ Participants will try to fill the map with post-it notes.

1.19. (5 min) Cluster similar ideas and discuss. Search for patterns and themes.

1.20. (5 $\mathrm{min})$ The last step will be presenting the new user experience to the note taker and I explaining how their solution solved the problems from the first exercise. 
1.21. Participants are thanked for their time and given a small break before the next exercise.

Materials needed include post it notes, a large whiteboard and markers.

Time is $30 \mathrm{~min}$

1.22. (5 min) Participants are guided to the computers that the activity will take place on.

1.23. A short introduction to the program and how to do certain functions is given.

1.24. Turn on recording equipment.

1.25. Ask the group if there are any questions before we get started and address those questions before moving on.

1.26. (15 min) Begin first section of the Feedback Grid activity

2.1. Explain that this activity is about gathering and organizing feedback from the other activities to keep building deeper. A quadrant map presented will have the following labels: things that worked, things to change, questions we still have, and new ideas to try.

2.2. Participants will use the provided equipment to fill in each quadrant with notes that are specific and give criticism.

2.3. Cluster similar ideas and discuss. Search for patterns and themes.

1.27. (5 $\mathrm{min})$ The last step will be presenting the patterns and themes that they found to the note taker and I.

1.28. (5 $\mathrm{min})$ The participants are given a post-test survey to complete (Appendix D).

1.29. Participants are thanked for their time and shown the way out.

Materials needed include a large format display, multiple devices and software to emulate the sticky notes. Participants could possibly run a web version on their own devices. 


\section{APPENDIX C}

Pre-test Survey 


\section{Collaborative Design Thinking in 2D and 3D \\ Pre-test Survey}

Group \#

1. What is your age?
$18-21$
$22-25$
$26-30$
$31-45$
$46+$

2. What career field are you interested/working in?

3. Have you ever participated in a design thinking activity before?

YES NO (skip to question 7)

4. If you answered YES to the question above, did you enjoy the activity?

YES (skip to question 6) NO

5. If you answered NO to the question above, why did you not enjoy the activity?

6. How many people participated in the activity?
$1-5$
$6-10$
$11-20$
$21-30$
$31+$

7. Do you see value in design thinking activities?

YES

NO

Why?

Thank you, please hand the survey back to the facilitator 


\section{APPENDIX D}

Post-test Survey 


\section{Collaborative Design Thinking in 2D and 3D \\ Post-test Survey}

Group \#

1. Did you enjoy the activity?

YES

NO

2. Why?

3. Which of the $\mathbf{3}$ different media did you prefer and why?

Physical Post-it

2D Digital Board

3D VR Environment

4. Did this activity change the way you see design thinking activities?

YES

NO

5. If yes, how did it change?

Thank you, please hand the survey back to the facilitator 


\section{APPENDIX E}

Recruitment Materials 


\section{Email Invitation: Adapting Collaborative Interactions Among Designers for use in 3D Virtual Reality}

Subject: Invitation to participate in a research project on collaborative design thinking in $2 \mathrm{D}$ and $3 \mathrm{D}$ space.

Dear Sir or Madam,

My name is Justin Chin and I am a master's student in the School of Industrial Design at Carleton University. I am working on a research project under the supervision of Prof. Lois Frankel.

I am writing to you today to invite you to participate in a study entitled "Adapting Collaborative Interactions Among Designers for use in 3D Virtual Reality". This study aims to find out how it is possible to increase interactions for collaboration and engagement among designers by giving them more access to decision making in a VR environment.

This study involves a 2-hour design thinking activity in a group setting that will take place in a mutually convenient, safe location. With your consent, the activity will be video-recorded. The videos will be anonymized and may be published.

The design activity consists of 3 parts where you will be given different materials to complete each task. In no specific order, you will be completing the tasks using post-it notes for one, a computer and digital board for another and a virtual reality headset for the remaining one. There is a possible risk of nausea if you have no experience with virtual reality. Please do not participate if you are susceptible to nausea.

While this project does not involve any professional and emotional risks, care will be taken to protect your identity should you wish to remain anonymous. This will be done by keeping requested responses anonymous and allowing you to request that certain responses not be included in the final project.

You will have the right to end your participation in the study at any time, for any reason, up until 2 weeks after completing the study. If you choose to withdraw, all the information you have provided will be destroyed. 
Light refreshments will be provided throughout the study.

All research data, including video-recordings and any notes will be encrypted. Any hard copies of data (including any handwritten notes or USB keys) will be kept in a locked cabinet at Carleton University. Research data will only be accessible by the researcher and the research supervisor.

The ethics protocol for this project was reviewed by the Carleton University Research Ethics Board, which provided clearance to carry out the research. Ethics Approval \# 108982 (Clearance expires on: July 31, 2019.)

If you have any ethical concerns with the study, please contact Dr. Bernadette Campbell, Chair, Carleton University Research Ethics Board-B (by phone at 613-520-2600 ext. 4085 or via email at ethics@carleton.ca). If you would like to participate in this research project, or have any questions, please contact me at justin.chin@carleton.ca.

Sincerely,

Justin Chin 


\section{Participate in a study on Adapting Collaborative Interactions Among Designers for use in 3D Virtual Reality}

To participate in this study you must be:

- Comfortable using computers

- At least 18 years old

- Comfortable in the English language with a group

- Have experience with design thinking activities

Please do not participate if you are susceptible to nausea

This is a 2 hour study. You will be asked to communicate with other participants to solve a design thinking exercise in three scenarios.

Light refreshments will be served.

You will have the right to end your participation in the study at any time, for any reason, up until 2 weeks after completing the study. If you choose to withdraw, all the information you have provided will be destroyed. The ethics protocol for this project has been reviewed and cleared by the Carleton University Research Ethics Board approval \#108982. If you have any ethical concerns with the study, please contact Dr. Bernadette Campbell, Chair, Carleton University Research Ethics Board-B (by phone at 613-520-2600 ext. 4085 or via email at ethics@carleton.ca).

Please contact the researcher, Justin Chin, for more details on this study at justin.chin@carleton.ca. 
APPENDIX F

Consent Form 


\section{Consent Form}

\section{Title: Adapting Collaborative Interactions Among Designers for use in 3D Virtual Reality}

Date of ethics clearance: To be determined by the REB (as indicated on the clearance form)

Ethics Clearance for the Collection of Data Expires: To be determined by the REB (as indicated on the clearance form)

I choose to participate in a study

on Adapting Collaborative Interactions Among Designers for use in 3D Virtual Reality. This study aims to find out how it is possible to increase interactions for collaboration and engagement among designers by giving them more access to decision making in a VR environment. The researcher for this study is Justin Chin in the School of Industrial Design at Carleton University. He is working under the supervision of Lois Frankel in the School of Industrial Design at Carleton University.

This study involves a 2 hour activity that will take place in a mutually convenient, safe location. With your consent, the activity will be video-recorded. Once the recording has been transcribed, the video-recording will be destroyed. You have the option of participating without being video-recorded.

The design activity consists of 3 parts where you will be given different materials to complete each task. In no specific order, you will be completing the tasks using post-it notes for one, a computer and digital board for another and a virtual reality headset for the remaining one. There is a possible risk of nausea if you have no experience with virtual reality. Please do not participate if you are susceptible to nausea.

While this project does not involve any professional and emotional risks, care will be taken to protect your identity. As this risk is expected to be minimal, I will take precautions to protect your identity. This will be done by keeping all responses anonymous and allowing you to request that certain responses not be included in the final project. Should you experience any distress during the interview, you will be provided with contact information for counseling services available nearby.

You have the right to end your participation in the study at any time, for any 
reason, up until 2 weeks after completing the study. You can withdraw by phoning or emailing the researcher or the research supervisor. If you decide to withdraw, the videos in which you participated in as part of the team will not be destroyed, but their participation will not be included in the data analysis or any presentations in which clips from the video are included. If your voice is still a part of the video clip, all the voices in that section will be altered to be unrecognizable.

Light refreshments will be served.

All research data, including video-recordings and any notes will be encrypted. Any hard copies of data (including any handwritten notes or USB keys) will be kept in a locked cabinet at Carleton University. Research data will only be accessible by the researcher and the research supervisor.

Once the project is completed, the digital data will be kept for 5 years after the study and destroyed. The paper data may be destroyed after submitting the final thesis document. The videos will be anonymized and may be published. (Electronic data will be erased and hard copies will be shredded.)

If you would like a copy of the finished research project, you are invited to contact the researcher to request an electronic copy which will be provided to you.

This research has been cleared by Carleton University Research Ethics Board-B Clearance \# 108982. Should you have any ethical concerns with the study, please contact Dr. Bernadette Campbell, Chair, Carleton University Research Ethics BoardB (by phone: 613-520-2600 ext. 4085 or by email: ethics@carleton.ca). For all other questions about the study, please contact the researcher.

\section{Researcher contact information:}

Name Justin Chin

Department: School of Industrial Design

Carleton University

Tel:

Email: justin.chin@carleton.ca

Do you agree to be video-recorded:

Signature of participant

Signature of researcher
Supervisor contact information:

Name Lois Frankel

Department: School of Industrial Design

Carleton University

Tel: 613-520-2600, ext. 5675

Email: lois.frankel@carleton.ca

Yes _ No

Date

Date

Page 2 of 2

This document has been printed on both sides of a single sheet of paper. Please retain a copy of this document for your records. 


\section{APPENDIX G}

Debriefing Script 


\section{DEBRIEFING}

\section{What are we trying to learn in this research?}

This research examines if it is possible to increase interactions for collaboration and engagement among designers by giving them more access to decision making in a virtual reality environment. The surveys you completed are being used to compared design thinking experience between groups using different methods. We are interested in learning if digital or virtual collaborative methods are beneficial for remote interactions and how they could be designed to accommodate that.

\section{Why is this important to scientists or the general public?}

It may result in guidelines that could support the design of software for collaborative 3D environments for decision making.

\section{What are our hypotheses and predictions?}

This research proposal hypothesizes that a virtual environment may improve interaction, collaboration and engagement, and ultimately improve the effectiveness specifically in remote design thinking methods.

1) Physical mediums like using post-it notes offer relatively little when it comes to collaborating with others in remote areas and make it harder to use other media like images.

2) 2D digital mediums like the software Mural, offer much more in terms of allowing remote collaborators to easily join and contribute, as well as giving the flexibility to add images and links.

3) 3D mediums like VR may take the digital space further by allowing presence of remote collaborators and closely mimicking the physical mediums while also allowing the flexibility of a digital medium.

\section{Where can I learn more?}

Design thinking is a complex subject and there are various ways that an exercise may be conducted. For more information you can check out the book 101 Design Methods by Vijay Kumar and Change by Design: How Design Thinking Transforms Organizations and Inspires Innovation by Tim Brown

Is there anything I can do if I found this experiment to be emotionally upsetting? Yes. If you feel any distress or anxiety after participating in this study, please feel free to contact the Carleton University Health and Counseling Services at: 613-520-6674, or the Distress Centre of Ottawa and Region at 613-238-3311 (http://www.dcottawa.on.ca).

\section{What if I have questions later?}

If you have any remaining concerns, questions, or comments about the experiment, please feel free to contact Justin Chin, at: justin.chin@carleton.ca (—), Dr. Lois Frankel, at: lois.frankel@carleton.ca (613-5202600, ext. 5675).

If you have any ethical concerns with the study, please contact Dr. Bernadette Campbell, Chair, Carleton University Research Ethics Board-A (by phone at 613-520-2600 ext. 2517 or via email at ethics@carleton.ca).

Thank you for participating in this research!

This research was cleared by Carleton University Research Board (A or B) Clearance \# 108982 (insert your ethics clearance number once obtained). 


\section{APPENDIX H}

IBM Design Thinking Field Guide 


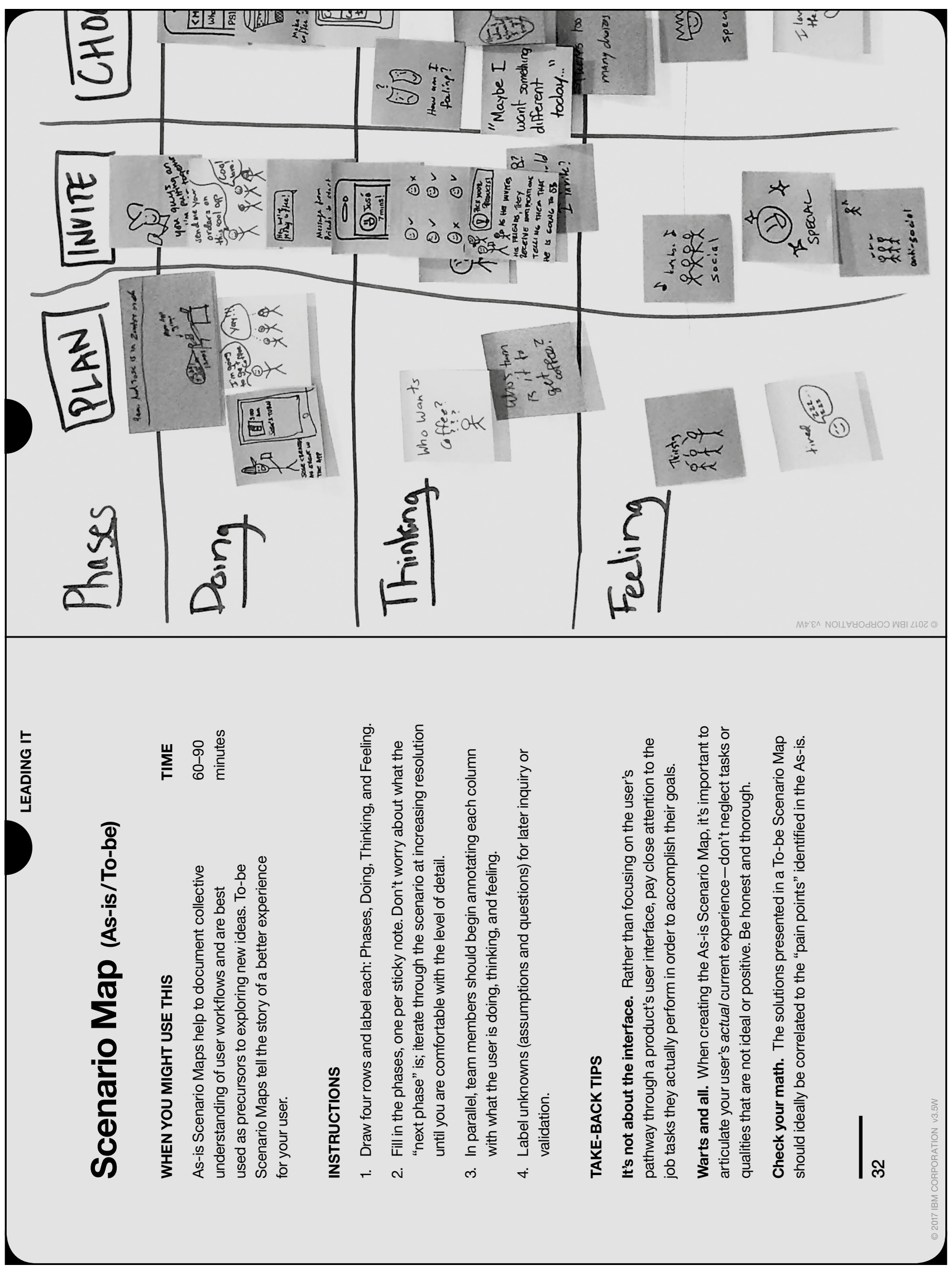




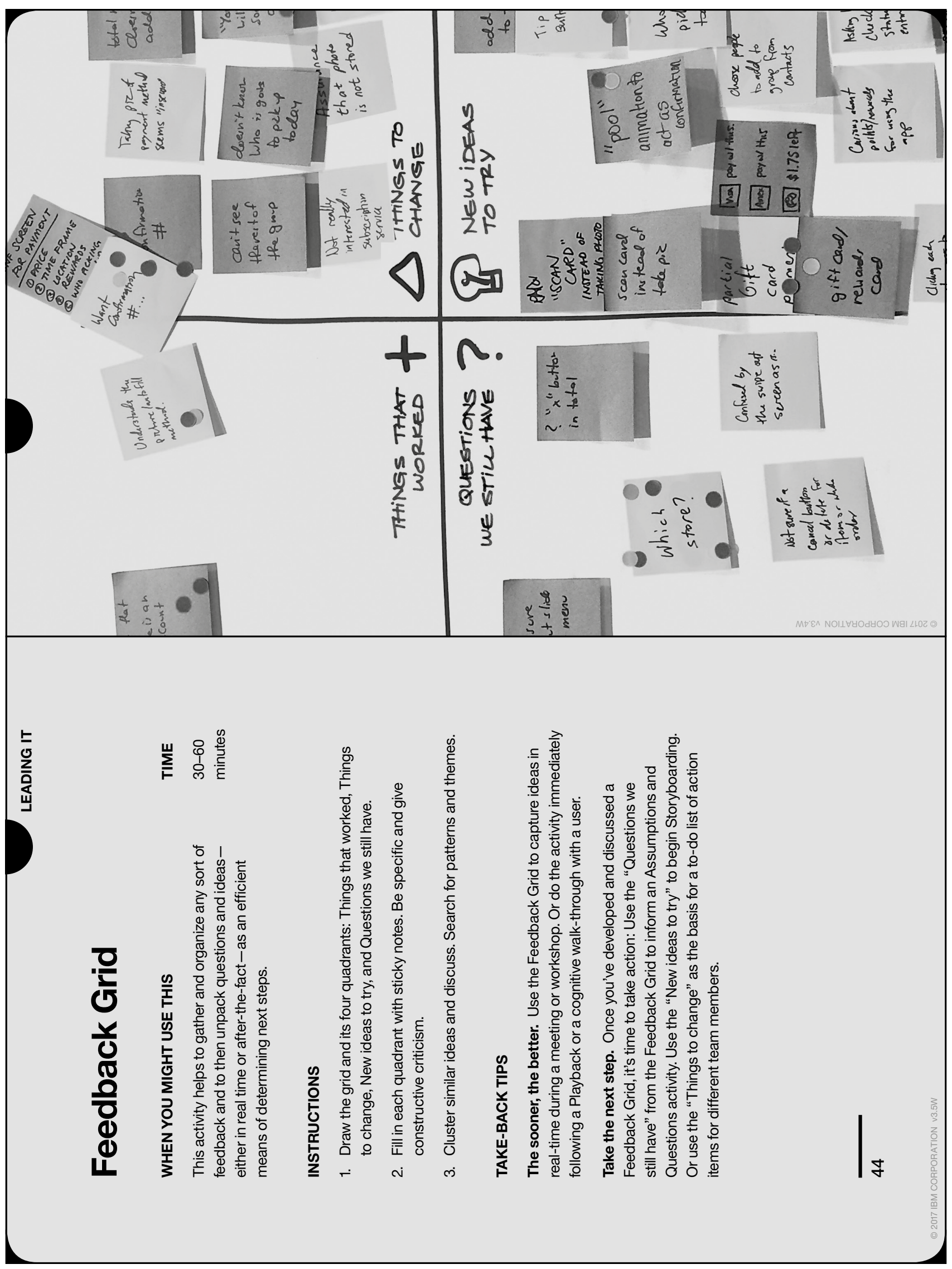


APPENDIX I

Codex 


\section{Look at Board}

Detailed description - when a participant looks at the group board that sticky notes are posted to, the digital board, or the virtual boards with notes.

Inclusion criteria - the participant looks at the board for information by reading what's posted.

Exclusion criteria - a quick glance without processing any information.

Typical exemplars $-\mathrm{n} / \mathrm{a}$.

Close, but no - less than a second glances that don't mean anything to the participant.

\section{Look at Others}

Detailed description - when participants look at each other either during talking or listening

Inclusion criteria - participant A looks at participant B while A is speaking to B. Participant A looks at participant B while B is speaking to A.

Exclusion criteria - participant A looks at participant $\mathrm{C}$ while neither of them are talking. Typical exemplars - a discussion between participant A and B occurs. They are both looking at each other while this happens.

Close, but no - participant A mentions something and looks at participant $\mathrm{C}$, but no other dialogue happens.

\section{Move Note}

Detailed description - every time a note or thought is moved around. Inclusion criteria - any note that is moved from its current position. Subsequent moves are considered a new move and not included in the previous recording.

Exclusion criteria - a note that is picked up but not moved.

Typical exemplars - note A moved from column A to column D, a sentence erased in VR and rewritten somewhere else. 
Close, but no - note picked up or erased but put back before moving to new location.

\section{Distracted}

Detailed description - when a participant is doing something off task. Depending on the medium this can vary what off-task thing they could be doing.

Inclusion criteria - playing with other objects like pens, paper, phone. In VR it could be drawing other off-topic stuff.

Exclusion criteria $-\mathrm{n} / \mathrm{a}$

Typical exemplars - participant A stops doing anything to check their phone. Participant C starts sketching/drawing off-topic ideas.

Close, but no $-\mathrm{n} / \mathrm{a}$

\section{Verbal Exchange}

Detailed description - when participants start a dialogue with each other and both parties are actively listening.

Inclusion criteria - participant A speaks to participant B and both respond to each other. This includes clarifications, verifications and agreement or disagreement.

Exclusion criteria - participant $\mathrm{C}$ says something aloud but no one responds.

Typical exemplars - participant A says why a note should be moved, participant B gives their opinion on why it should stay and a discussion occurs.

Close, but no - n/a. 